\title{
Inhaltliche Erschließung von Altbeständen in allgemeinen Bibliothekskatalogen. Bestandsaufnahme und Entwicklungsmöglichkeiten.
}

\author{
Bachelorarbeit \\ im Studiengang Bibliotheks- und Informationsmanagement \\ der \\ Hochschule der Medien Stuttgart
}

Hannes Schwarzendorfer

Erstprüferin:

Heidrun Wiesenmüller M.A.

Zweitprüfer:

Prof. Bernhard Hütter

Bearbeitungszeitraum: 01. März 2007 bis 01 . Juni 2007 Überarbeitete Fassung, Februar 2008

Stuttgart, 1. Juni 2007 


\section{Erklärung}

Hiermit erkläre ich, dass ich die vorliegende Bachelorarbeit selbständig angefertigt habe. Es wurden nur die in der Arbeit ausdrücklich benannten Quellen und Hilfsmittel benutzt. Wörtlich oder sinngemäß übernommenes Gedankengut habe ich als solches kenntlich gemacht.

Ort, Datum

Unterschrift 


\section{Kurzfassung}

Das Thema der hier vorliegenden Bachelorarbeit ist die inhaltliche Erschließung von Altbeständen in allgemeinen Bibliothekskatalogen. Nach einer allgemeinen Einführung und der Betrachtung der Entwicklung in den letzten Jahrzehnten wird die ErschlieBungssituation in mehreren Bibliotheken Deutschlands sowie in der Österreichischen Nationalbibliothek vorgestellt. Anhand der Systematikgruppe IV.18 (Geschichte der skandinavischen Reiche) der Oettingen-Wallersteinschen-Bibliothek folgt ein ausführlicher Überblick über den momentanen Stand der Sacherschließung innerhalb des Dreiländerkatalogs sowie innerhalb der deutschen Verbundkataloge und den Katalogen der Staatsbibliothek Berlin und wiederum der Österreichischen Nationalbibliothek. Diese werden mit Hilfe des Karlsruher Virtuellen Katalogs näher untersucht und ausgewertet. Im Anschluss daran wird versucht, Möglichkeiten einer zukünftigen sachlichen Altbestandserschließung aufzuzeigen.

Schlagwörter: Altbestand, Altes Buch, Bestandserschließung, Inhaltserschließung, retrospektive Konversion, Sachkatalog

\section{Abstract}

The topic of this bachelor thesis is subject indexing of old printed books in general library catalogues. After a general introduction and the consideration of the development in the last decades the indexing situation in several libraries of Germany as well as in the Austrian National Library will be presented. On the basis of the classification number IV.18 (History of Scandinavia) of the Oettingen-Wallersteinsche library follows a detailed overview of the current situation of subject indexing within the Tri-National Catalogue as well as within the german union catalogues and the online catalogues of the Berlin State Library and again of the Austrian National Library. These catalogues are analysed and evaluated with the help of the Karlsruhe Virtual Catalog. Afterwards it will be tried to indicate possibilities of a future subject indexing of old printed books.

Keywords: old stock, old printed books, stock exploitation, subject indexing, retrospective conversion, subject catalogue 


\section{Inhaltsverzeichnis}

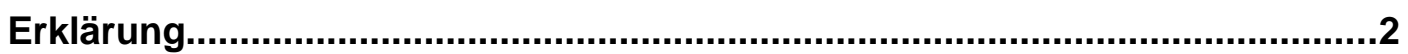

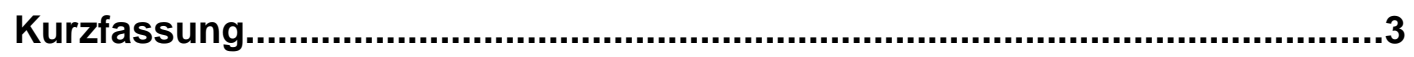

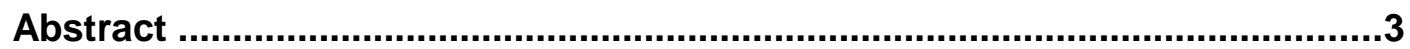

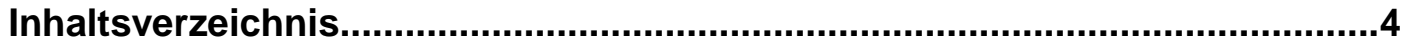

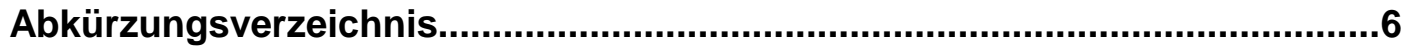

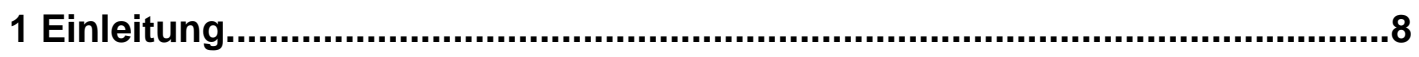

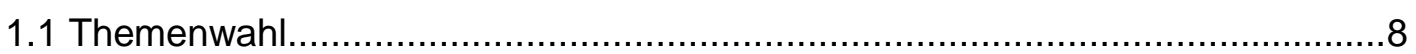

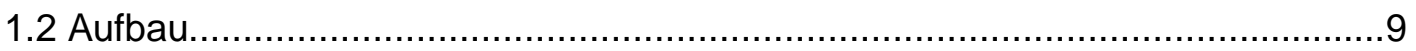

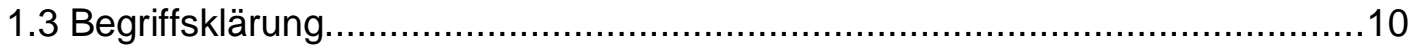

2 Grundlagen der inhaltlichen Altbestandserschließung..............................12

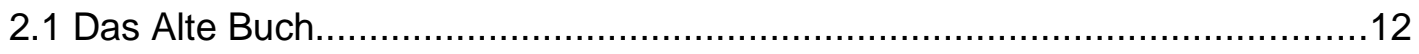

2.2 Inhaltliche Altbestandserschließung...................................................... 13

3 Die Erschließungssituation im Überblick...................................................16

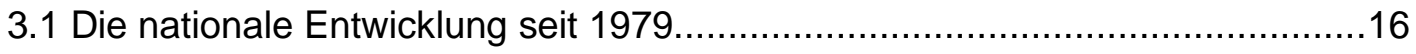

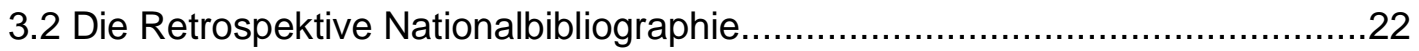

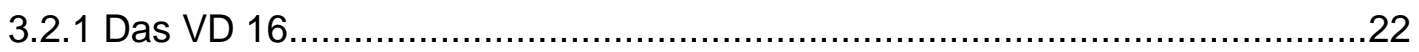

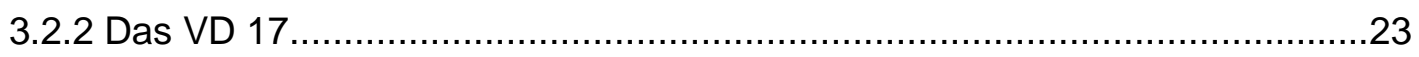

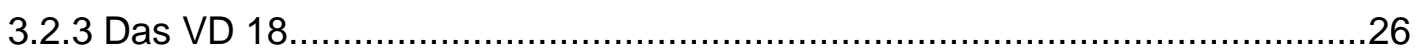

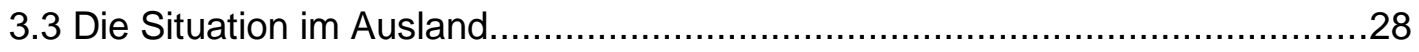

4 Die Erschließungssituation in ausgewählten Bibliotheken .........................32

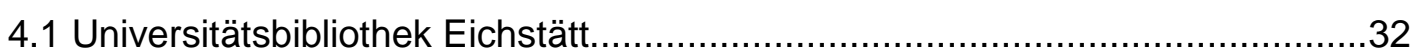

4.2 Staats- und Universitätsbibliothek Hamburg................................................33

4.3 Staats- und Universitätsbibliothek Göttingen................................................34

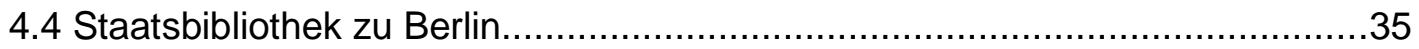

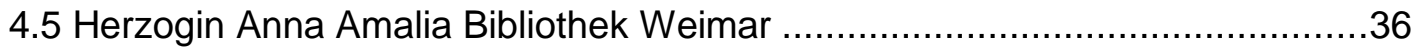

4.6 Herzog August Bibliothek Wolfenbüttel................................................38

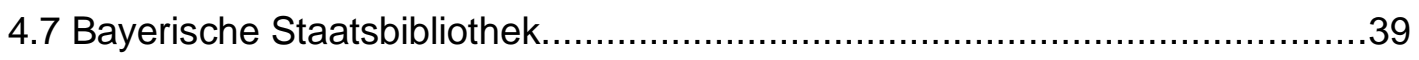

4.8 Österreichische Nationalbibliothek....................................................... 40

5 Die Erschließungssituation innerhalb allgemeiner Bibliothekskataloge.....42

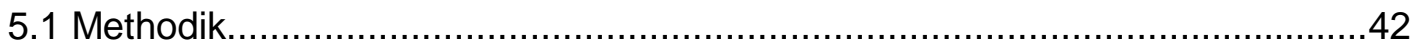

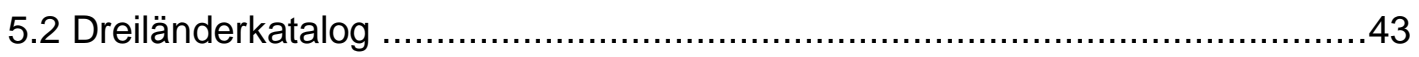

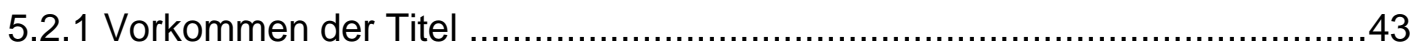




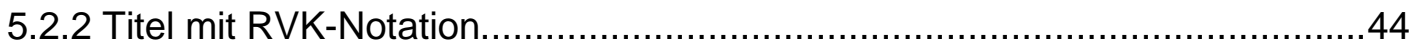

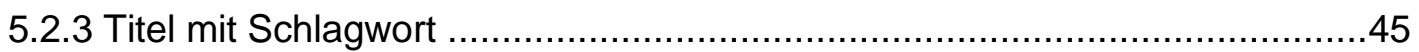

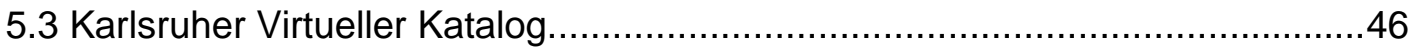

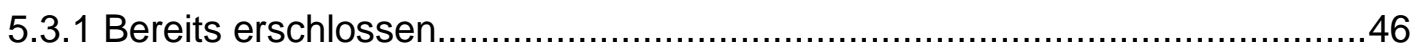

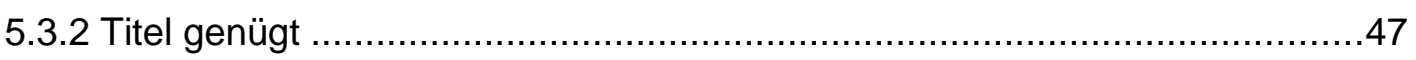

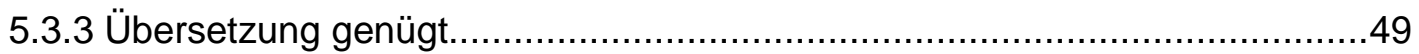

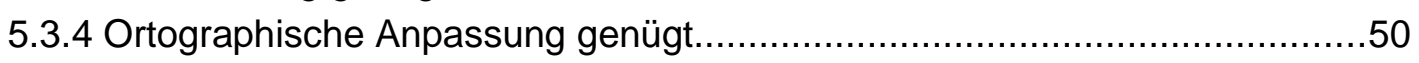

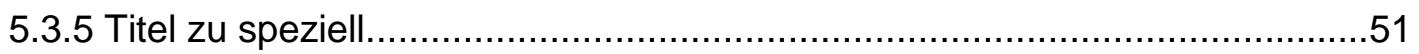

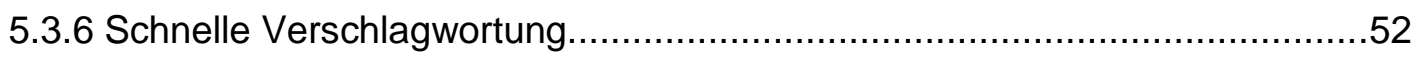

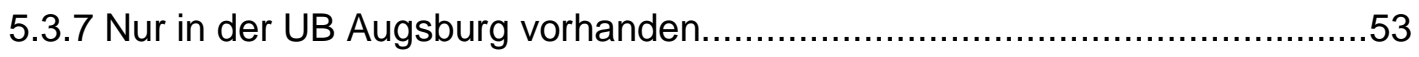

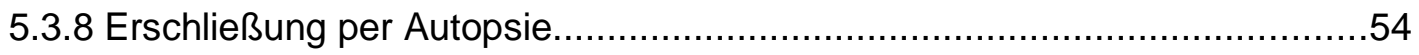

5.3.9 Betrachtungen einzelner Verbünde und Bibliotheken...............................56

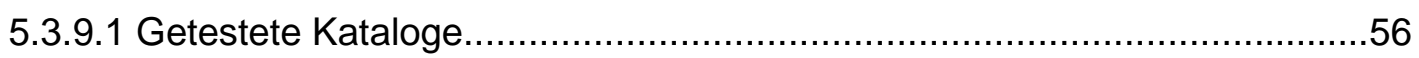

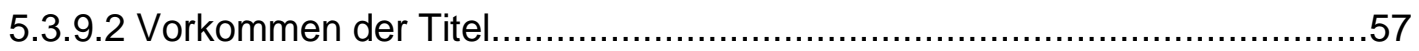

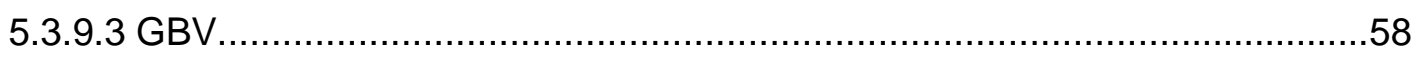

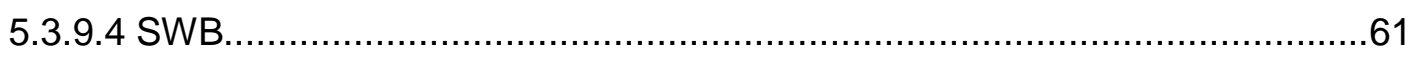

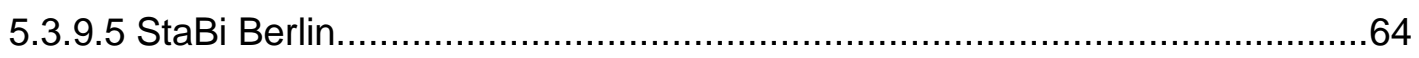

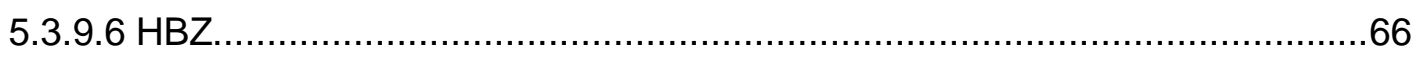

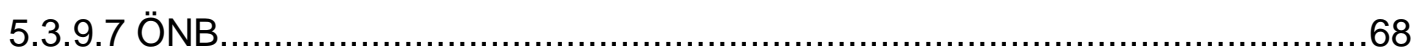

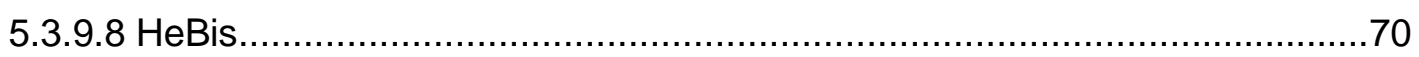

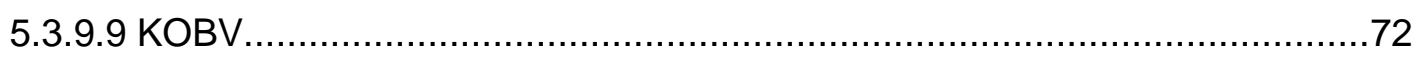

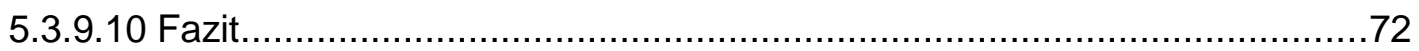

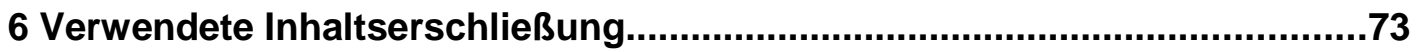

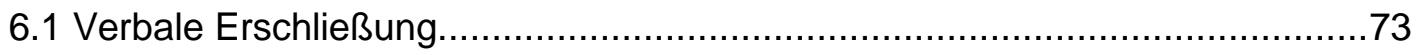

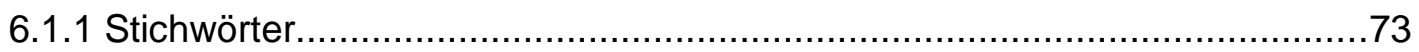

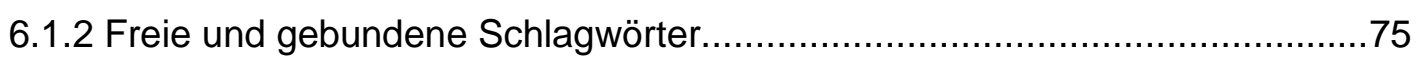

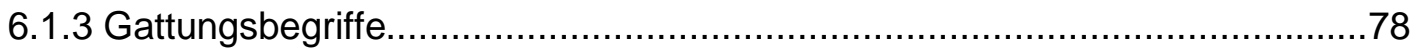

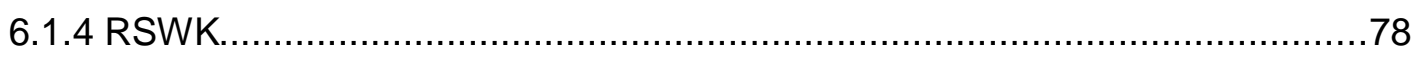

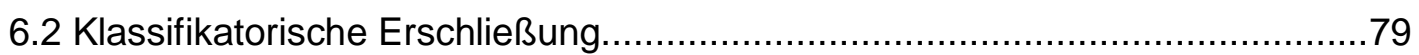

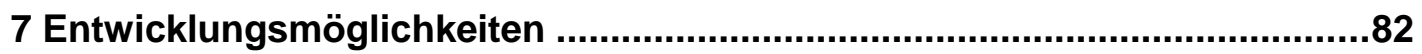

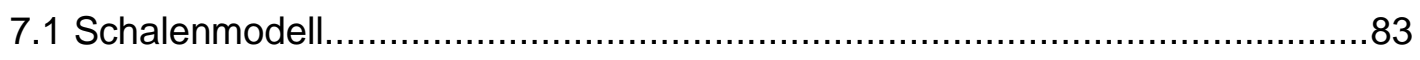

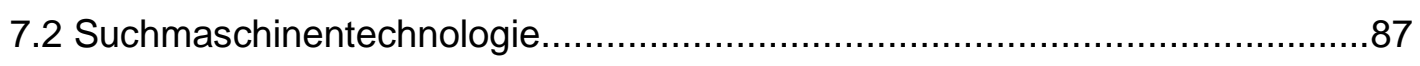

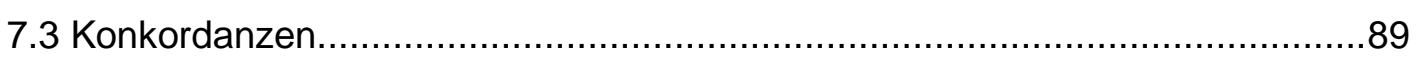

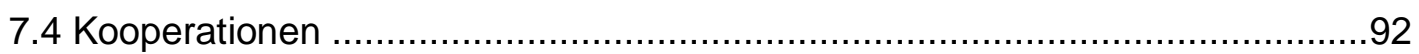

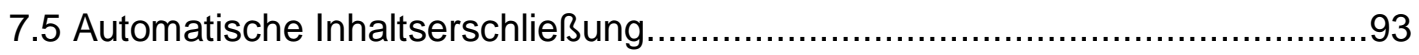

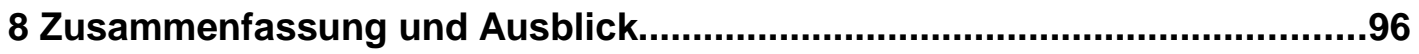

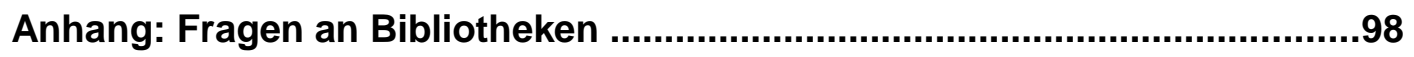

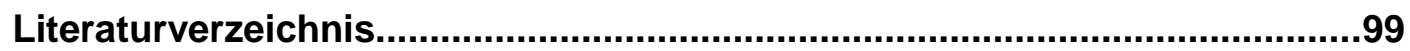




\section{Abkürzungsverzeichnis}

\begin{tabular}{|c|c|}
\hline AAD & Arbeitsgemeinschaft Alte Drucke beim GBV \\
\hline AG SDD & Arbeitsgemeinschaft Sammlung Deutscher Drucke \\
\hline ALA & American Library Association \\
\hline ARK & Alter Realkatalog \\
\hline ABE & Altbestandserschließung \\
\hline BK & Basisklassifikation \\
\hline BSB & Bayerische Staatsbibliothek \\
\hline BVB & Bayerischer Bibliotheksverbund \\
\hline DDC & Dewey Decimal Classification \\
\hline DFG & Deutsche Forschungsgemeinschaft \\
\hline DLK & Dreiländerkatalog \\
\hline ECSTC & Eighteenth Century Short Title Catalogue \\
\hline ESTC & English Short Title Catalogue \\
\hline GBV & Gemeinsamer Bibliotheksverbund \\
\hline GV & Gesamtverzeichnis des deutschsprachigen Schrifttums \\
\hline HABW & Herzog August Bibliothek Wolfenbüttel \\
\hline HAAB & Herzogin Anna Amalia Bibliothek Weimar \\
\hline HBZ & Hochschulbibliothekszentrum des Landes Nordrhein-Westfalen \\
\hline HeBIS & HessischesBibliotheksInformationsSystem \\
\hline KVK & Karlsruher Virtueller Katalog \\
\hline KOBV & Kooperativer Bibliotheksverbund Berlin-Brandenburg \\
\hline $\mathrm{LCSH}$ & Library of Congress Subject Headings \\
\hline LOC & Library Of Congress \\
\hline ÖNB & Österreichische Nationalbibliothek \\
\hline OPAC & Online Public Access Catalogue \\
\hline PND & Personennamendatei \\
\hline RSWK & Regeln für den Schlagwortkatalog \\
\hline RVK & Regensburger Verbundklassifikation \\
\hline SDD & Sammlung Deutscher Drucke \\
\hline
\end{tabular}


StaBi Staatsbibliothek zu Berlin - Preussischer Kulturbesitz

STCN Short Title Catalogue Netherlands

STCV Short Title Catalogue Vlandeeren

SB Stadtbibliothek

SUB Staats- und Universitätsbibliothek

SUBG Staats- und Universitätsbibliothek Göttingen

SW Schlagwort

SWB Südwestdeutscher Bibliotheksverbund

SWD Schlagwortnormdatei

UB Universitätsbibliothek

UBA Universitätsbibliothek Augsburg

ULBH Universitäts- und Landesbibliothek Sachsen-Anhalt Halle

VD 16 Verzeichnis der im deutschen Sprachbereich erschienenen Drucke des 16. Jh

VD 17 Verzeichnis der im deutschen Sprachraum erschienenen Drucke des 17. Jh

VD 18 Verzeichnis der im deutschen Sprachraum erschienenen Drucke des 18. Jh 


\section{$1 \quad$ Einleitung}

\subsection{Themenwahl}

Dieser Arbeit ging ein Projekt voraus, welches im Zeitraum von September 2006 bis Februar 2007 an der Universitätsbibliothek Augsburg durchgeführt wurde.

Anhand der Bestände der Öttingen-Wallersteinschen Bibliothek wurden „Möglichkeiten zur Verbesserung der Inhaltlichen Erschließung von Altbeständen“ untersucht.

Während der Bearbeitung des Projektes fiel schnell auf, dass es zwar zahlreiche Literatur über die Erschließung von Bibliotheksbeständen gibt, diese jedoch meist die allgemeine, d.h. die formale Erschließung und die damit verbundene Bereitstellung der Bestände behandelt. Literatur über die sachliche bzw. inhaltliche Erschließung von Bibliotheksgut ist schon ein wenig seltener. Sehr selten ist jedoch bedauerlicherweise Fachliteratur über die inhaltliche Erschließung von Altbeständen. Abhandlungen und Aufsätze über dieses Thema findet man nur sehr schwer, und wenn, dann wird die Thematik meist nur gestreift und die konkrete Erschließung wird nur als Teilaspekt behandelt. Ein Standardwerk oder auch nur eine ausführliche Monographie über den angesprochenen Sachverhalt findet sich schon gar nicht. So musste die Grundlagenliteratur für das Projekt und ebenso für die an diese Thematik anschließende, hier vorliegende Arbeit aus verschiedenen Teilbereichen des Bibliothekswesens zusammengetragen werden. Zudem musste vieles eigenständig recherchiert und bei den jeweiligen verantwortlichen Personen persönlich nachgefragt werden.

Bei Überlegungen über die Sacherschließung von Altbeständen muss man natürlich die Frage einbeziehen, für welchen Zweck diese denn erschlossen werden sollen. So können Bestände entweder für die Nutzung innerhalb spezieller Altbestands-Nachweissysteme wie dem Verzeichnis der im deutschen Sprachraum erschienenen Drucke des 17. Jh. erschlossen und dort gezielt an den Anforderungen von Wissenschaftlern ausrichtet werden; oder sie können für die Nutzung innerhalb allgemeiner Bibliothekskataloge, und somit neben den Wissenschaftlern auch für allgemeine Nutzer erschlossen werden. Diese Arbeit hat die allgemeine Nutzung zum Thema. Da jedoch Altbestände und deren formale und inhaltliche Erschließung ein sehr spezieller Bereich innerhalb der Bibliothekswissenschaft sind, muss auch ein Blick auf die Entwicklung von überregionalen Altbestandserschließungsprojekten und deren jeweilige Erschließungspraxis geworfen werden.

Neben diesen allgemeinen Unternehmungen gab es in den vergangenen Jahren selbstverständlich auch etliche Projekte zur Erschließung einzelner Spezialbestände, so z.B. für Historische Karten, Einblattdrucke und Illustrationen. Jedoch wird dieser Teil der Thematik in der vorliegenden Arbeit nicht weiter untersucht, da dies den Rahmen einer Bachelorarbeit sprengen würde. 
Des Weiteren wären aus den vorhandenen Daten noch zahlreiche weitere, durchaus sinnvolle Auswertungen möglich gewesen, jedoch musste auch hier ausgewählt und eingegrenzt werden, damit der formale Rahmen eingehalten werden konnte.

\subsection{Aufbau}

Zu Beginn der Arbeit wird auf die allgemeinen Grundlagen der AltbestandserschlieBung eingegangen. Zentrale Begriffe werden definiert. Sodann wird über verschiedene Projekte informiert, die seit 1979 die Altbestandserschließung zum Ziel hatten, wenn auch meist nicht inhaltlich sondern nur rein formal. Nähere Betrachtungen verdienen die jüngsten Anstrengungen zum Aufbau einer retrospektiven Nationalbibliographie, die sich aus den beiden bereits verwirklichten Projekten Verzeichnis der im deutschen Sprachbereich erschienenen Drucke des 16. Jh. (VD 16) und Verzeichnis der im deutschen Sprachraum erschienenen Drucke des 17. Jh. (VD 17), sowie eines geplanten Verzeichnis der im deutschen Sprachraum erschienenen Drucke des 18. Jh. (VD 18) zusammensetzt. Nachdem auf die deutsche Situation ausführlich eingegangen wurde, wird noch ein kurzer Blick ins Ausland geworfen. Dabei wird die Sacherschließungspraxis des Short Title Catalogue Netherlands (STCN), des Short Title Catalogue Vlandeeren (STCV), des English Short Title Catalogue (ESTC) und der Library of Congress (LOC) näher erläutert.

Anschließend wird der momentane Stand der inhaltlichen Erschließung von Altbeständen in einzelnen Bibliotheken und Verbünden des deutschsprachigen Raumes untersucht.

Zuerst werden einige Bibliotheken betrachtet. Dazu wurden die einzelnen Bibliotheken gebeten, einige Fragen zu ihrer jeweiligen Situation bezüglich der Sacherschließung ihrer Altbestände zu beantworten. Zusätzlich wurde, soweit vorhanden, Fachliteratur ausgewertet. Weitere wertvolle Hinweise gab es auf den jeweiligen Webseiten der einzelnen Bibliotheken, auf denen teils sehr ausführlich deren Erschließungspraxis dargelegt wird.

Nach dieser allgemeinen Begutachtung einzelner Bibliotheken folgt eine Untersuchung zur genauen Erschließungssituation im Dreiländerkatalog (DLK) und im Karlsruher Virtuellen Katalog (KVK) anhand des Bestandes der Systematikgruppe IV.18 (Geschichte der skandinavischen Reiche) der Oettingen-Wallersteinschen Bibliothek der UB Augsburg (UBA). Diese Bestandsgruppe wurde aufgrund ihrer geeigneten Anzahl von 209 Titeln ausgewählt. Die Titelmenge ist somit einerseits groß genug, um bei der Auswertung eine gewisse Signifikanz zu gewähren und andererseits nicht zu groß, so dass die Titelzahl überschaubar ist und die Auswertungen vom zeitlichen Aufwand her durchführbar sind. Näher betrachtet wird im Dreiländerkatalog, welche Titel bereits mit Schlagwörtern (SW) oder mit der Regensburger Verbundklassifikation (RVK) erschlossen sind. Zusätzlich wird überprüft, wie viele Titel dieser Systematikgruppe sich in den Katalogen der Niedersächsischen Staats- und Universitätsbibliothek Göttingen (SUBG), der Staatsbibliothek zu Berlin - Preussischer Kulturbesitz (StaBi Berlin) und 
der Universitäts- und Landesbibliothek Sachsen-Anhalt Halle (ULBH) befinden. Mit Hilfe des KVK wurden zuerst verschiedene allgemeine Gegebenheiten untersucht. Anschließend wird sowohl in den deutschen Verbundkatalogen als auch in den Katalogen zweier Bibliotheken geprüft, wie viele Titel dieser Systematikgruppe vorhanden und in welcher Qualität diese erschlossen sind. Bei den beiden Bibliotheken handelt es sich sowohl um den Katalog 1501-1929 der Österreichischen Nationalbibliothek (ÖNB) als auch um den Katalog der Staatsbibliothek zu Berlin. Diese wurden zu den Verbünden hinzugenommen, da sie direkt über den KVK recherchiert werden können. Folgende Kataloge wurden ausgewertet:

- Gemeinsamer Bibliotheksverbund

GBV

- Hochschulbibliothekszentrum des Landes Nordrhein-Westfalen

HBZ

- HessischesBibliotheksInformationsSystem

HeBIS

- Kooperativer Bibliotheksverbund Berlin-Brandenburg

KOBV

- Südwestdeutscher Bibliotheksverbund

SWB

Der Bayerische Verbundkatalog (BVB) wurde zu dieser Auswertung nicht hinzugezogen, da er durch die UB Augsburg und seine Beteiligung am Dreiländerkatalog schon zu einem gewissen Grad abgedeckt ist. Im Anschluss an diese Untersuchungen wird ein Resümee bezüglich der verwendeten Mittel zur Erschließung der jeweiligen Bestände gezogen und diese auf ihre Tauglichkeit hinterfragt. Zum Schluss wird versucht, Möglichkeiten aufzuzeigen, wie man einen verbesserten sachlichen Zugang zu Altbeständen möglichst effizient, d.h. zeit-, personal- und kostensparend, erreichen könnte.

\subsection{Begriffsklärung}

Nach diesem Überblick über den Aufbau der vorliegenden Arbeit gilt es, kurz die grundlegenden Begriffe des Themas zu definieren. So geht es bei der SacherschlieBung (Inhaltlichen Erschließung) ${ }^{1}$ „im Gegensatz zur Formalerschließung, bei der ein Buch nach seinen formalen Elementen (z.B. Verfassername, Sachtitel) beschrieben und verzeichnet wird, [...] um die inhaltliche Beschreibung und Erschließung von Literatur." Dabei „ist zu unterscheiden zwischen der verbalen Sacherschließung, die hauptsächlich natürlich-sprachliche Bezeichnungen (Schlagwörter, Stichwörter) verwendet, und der klassifikatorischen Sacherschließung, die vorwiegend auf Klassifikationssystemen mit hierarchisch geordneten Systemstellen (Notationen) beruht. “2

Eine solche Klassifikation ist z.b. die Regensburger Verbundklassifikation, die u.a. flächendeckend in bayerischen Hochschulbibliotheken verwendet wird. Das zentrale An-

1 Sacherschließung (bzw. sachliche Erschließung) und Inhaltserschließung (bzw. inhaltliche Erschließung) werden in dieser Arbeit als synonyme Begriffe behandelt.

2 Hacker, Rupert (2000): Bibliothekarisches Grundwissen. 7., neu berabeitete Auflage. München 
liegen von Sacherschließung gilt der Ermöglichung des Auffindens von Monographien, Zeitschriftenaufsätzen und sonstigen Dokumenten auch ohne Kenntnis der bibliographischen und formalen Daten. ${ }^{3}$ Als weitere Mittel zur inhaltlichen Erschließung dienen Stichwörter, Gattungsbegriffe, Inhaltsverzeichnisse und Abstracts.

Im Laufe der Arbeit wird man mehrere Male auf die Begriffe Retrokonversion und Retrokatalogisierung stoßen. Dabei meint Retrokonversion bzw. Retrospektive Konversion „eine Sonderform der Erschließung. [...] Sie setzt voraus, dass bereits ErschlieBungsarbeit geleistet wurde, deren Wert so hoch ist, dass das Ergebnis erhaltungswürdig ist. Diesen Erhalt garantiert die Konversion und fügt je nach Verfahren einen Mehrwert hinzu. [...] Retrokonversion heißt das Umsetzen konventioneller Erschließung in elektronischer Form. ${ }^{44}$ Retrokonversion bedeutet also die Umwandlung eines Informationsmediums in ein anderes Informationsmedium, wobei dieses meist höherwertig ist, z.B. physisch stabiler. So u.a. die Umwandlung eines Kartenkatalogs in einen OnlineKatalog. ${ }^{5}$ Die Retrokatalogisierung (Rekatalogisierung, Retrospektive Katalogisierung) hingegen „beginnt [...] wieder bei der Vorlage selbst, der Ausgabe eines Werkes, das einer erneuten Erschließung - nach den aktuellen Regeln und mit den neuesten Verfahren, gewöhnlich also maschinenlesbar - ohne Berücksichtigung der bereits vorliegenden Erschließung zugeführt wird. "“6 Retrokatalogisierung meint also die erneute Katalogisierung alter Bestände.

Eine genaue Betrachtung und eine zeitliche Eingrenzung von Altbeständen erfolgt anschließend in Kap. 2.1. anhand des Begriffes des Alten Buches. Weitere Definitionen zu relevanten Benennungen folgen im Laufe der Arbeit.

3 Vgl. dazu Haller, Klaus; Fabian, Gudrun (2004): Bestandserschließung. In: Frankenberger, Rudolf; Haller, Klaus (Hg.): Die moderne Bibliothek. Ein Kompendium der Bibliotheksverwaltung. Herausgegeben von Rudolf Frankenberger und Klaus Haller. München: Saur, S. 222261, hier S.227

4 Haller, Klaus; Fabian, Gudrun (2004), hier S. 253

5 Löffler, Karl; Umstätter, Walther; Wagner-Döbler, Roland (2005): Einführung in die Katalogkunde. Vom Zettelkatalog zur Suchmaschine. Dritte Auflage des Werkes von Karl Löffler. Völlig neu bearbeitet von Walther Umstätter und Roland Wagner-Döbler. Stuttgart: Hiersemann, hier S. $41 \mathrm{f}$.

6 Vgl. Haller, Klaus; Fabian, Gudrun (2004), hier S. 254 


\section{Grundlagen der inhaltlichen Altbestandserschließung}

\subsection{Das Alte Buch}

Unter dem Begriff des Alten Buches ${ }^{7}$ werden im Allgemeinen jene Druckwerke verstanden, die zwischen der Erfindung des Buchdrucks Mitte des 15. Jahrhunderts durch Gutenberg und dem Beginn der Industrialisierung des Buchdrucks, eingeleitet durch die Erfindung der Schnellpresse zu Beginn des 19. Jahrhunderts durch Friedrich König, hergestellt wurden. Jedoch existiert weder eine allgemeingültige Definition noch eine einheitlich anerkannte Zeitgrenze. Eine der wenigen Versuche einer Eingrenzung, auf welche man sich auch heute noch häufig bezieht, ${ }^{8}$ stammt von Paul Raabe. ${ }^{9}$ So endet diese Phase, die erste Phase der buchgeschichtlichen Entwicklung, für ihn um 1830. Das Buch aus jener Zeit sei „handgesetzt“ und „auf geschöpftem Papier von Hand gedruckt“. Es „zeichne sich durch eine niedrige Auflage aus“, meist weniger als „1000 Exemplare“ eines Werkes, sowie durch eine „individuelle Qualität“. Deshalb handele es sich um Unikate, um „Individualitäten“; in dieser Zeit „von Dubletten zu sprechen“ sei "gewagt“. Die zweite Phase dauert für Raabe von 1830-1950. Er spricht in diesem Zeitraum vom ,,älteren Buch“. Darunter fallen „Bücher aus der Frühzeit des Maschinenzeitalters“, „Drucke der Jahrhundertwende“, „die Bücher des Expressionismus und der zwanziger Jahre“. Dazu gesellen sich noch Bücher aus „der Nazizeit und selbst (aus) der Nachkriegszeit.“ Da es „meist in höheren Auflagen hergestellt“ sei, verliere es seine Seltenheit und Individualität und sei nicht so „unikal“ wie die Bücher der ersten Phase. Aufgrund dieser Merkmale sei das Buch wissenschaftlich weniger interessant und kulturell weniger wertvoll, jedoch mache es die ,inhaltliche Bedeutung in Verbindung mit der zeitbezogenen äußeren Gestalt“ häufig interessant für eine „von Werten ausgehenden Forschung“. Das „alte Buch“ ist für inn also „die Summe des Gedruckten bis 1830 und die Summe der inhaltlich und äußerlich wesentlichen Bücher aus der Zeit der maschinellen Herstellung des Buches zwischen 1830 und 1950."

Da sich die mit beweglichen Lettern hergestellten Drucke des 15. Jahrhunderts, die sogenannten Wiegendrucke oder Inkunabeln, nochmals in zahlreichen Punkten von den

7 Das Alte Buch, das Historische Buch, der Altbestand (bzw. die Altbestände) und der historische Bestand (bzw. die historischen Bestände) werden in dieser Arbeit ebenfalls als synonyme Begriffe behandelt.

8 So z.B. Brinkhus, Gerd (2006): Der historische Altbestand in der modernen Bibliothek. Forum Bestandserhaltung. Online verfügbar unter http://www.uni-muenster.de/Forum-Bestandserhaltung/grundlagen/allg-brinkus.html, zuletzt aktualisiert am 31.05.2006, zuletzt geprüft am 16.02.2008.

9 Vgl. Raabe, Paul (1979): Das alte und kostbare Buch - eine bibliothekarische Zukunftsaufgabe. In: Fuhlrott, Rolf; Haller, Bertram (Hg.): Das Buch und sein Haus. Gerhard Liebers gewidmet zur Vollendung des 65. Lebensjahres am 23. Mai 1979. Wiesbaden: Reichert, S. 164-188, hier S. $167 \mathrm{ff}$. 
Drucken ab 1501 unterscheiden, ${ }^{10}$ werden diese meist separat betrachtet und auch separat behandelt. So sind Inkunabeln häufig in eigenen Spezialkatalogen nachgewiesen, z.B. im Gesamtkatalog der Wiegendrucke ${ }^{11}$ und im Inkunabelkatalog INKA ${ }^{12}$.

Aufgrund dieser Unterschiede wurden bei den hier durchgeführten Überlegungen die Drucke vor 1500 ebenfalls nicht miteinbezogen. Die entgegengesetzte Grenze wurde um 1850 gesetzt. Raabe spricht beim Alten Buch von 1830, um jedoch eine größere Menge an Titeln zu bekommen, erschien 1850 sinnvoller, wobei die Drucke aus der zweiten Phase bis 1950 bewusst weggelassen wurden.

\subsection{Inhaltliche Altbestandserschließung}

„Wie einschlägige Untersuchungen und die Erfahrung von Bibliotheksmitarbeitern zeigen, gehört ungefähr die Hälfte der Anfragen an Bibliothekskataloge in die Kategorie der thematischen oder sachlichen Suche. “13

Die Wichtigkeit und Notwendigkeit einer inhaltlichen Erschließung für Bibliotheksbestände ist unumstritten. „Eine effiziente Sacherschließung“ ist eine existenzielle Grundaufgabe und "gehört zum Selbstverständnis qualitätsbewusster und an Wirtschaftlichkeit orientierter Bibliotheken." ${ }^{14}$ Ebenso wie für aktuelle Literatur gilt dies selbstverständlich auch für die historischen Bestände, die trotz starker Verluste im Zweiten Weltkrieg noch zahlreich in Bibliotheken des deutschen Sprachraums vorhanden sind. Beschäftigt man sich näher mit dieser Thematik, stellt man jedoch schnell fest, dass die Zufriedenheit mit der aktuellen Situation bezüglich der Sacherschließung alter Bestände seitens der Bibliotheken und vor allem seitens der Wissenschaft sehr gering ist, das Interesse daran hingegen sehr groß. So z.B. bei Arnold: „Sehr schnell wird [...] in

10 Natürlich ist der Übergang fließend und nicht an einem bestimmten Datum festzumachen. Die ersten Drucker zu Beginn des Buchdrucks mit beweglichen Lettern orientierten sich jedoch noch stark an den Handschriften. So wurden die Initialen und Rubrizierungen per Hand eingefügt. Die Holzschnitte und Ornamente wurden ebenfalls per Hand koloriert. Wiegendrucke enthalten genau wie Handschriften sowohl ein „Incipit“ und ein „Explicit“ als auch ein Kolophon. Das Titelblatt kam erst am Ende der Inkunabelzeit auf. Auch Inhaltsverzeichnisse gab es noch nicht, ebenso keine Seitenzählung. Zudem wurden weiterhin zahlreiche Abbreviaturen und Ligaturen verwendet. Vgl. u.a. Funke, Fritz (2006): Buchkunde. Die historische Entwicklung des Buches von der Keilschrift bis zur Gegenwart. 6., überarb. und erg. Aufl., Sonderausgabe. Wiesbaden: Albus im VMA-Verlag, hier S. 115

11 Suckow, Ninon; Klarkowski, Werner (2008): Gesamtkatalog der Wiegendrucke. Staatsbibliothek zu Berlin (SBB). Online verfügbar unter http://www.gesamtkatalogderwiegendrucke.de/, zuletzt aktualisiert am 16.01.2008, zuletzt geprüft am 16.02.2008.

12 INKA (2008). Online verfügbar unter http://www.inka.uni-tuebingen.de/?bib=stu, zuletzt geprüft am 16.02.2008.

13 Mittelbach, Jens; Probst, Michaela (2006): Maschinelle Indexierung in der Sacherschließung wissenschaftlicher Bibliotheken. In: Bibliothek. Forschung und Praxis, Jg. 30, H. 2, S. 168176. Online verfügbar unter http://www.bibliothek-saur.de/2006_2/168-176.pdf, zuletzt geprüft am 23.02.2008, hier S. 168

14 Flachmann, Holger (2004): Zur Effizienz bibliothekarischer Inhaltserschließung: Allgemeine Probleme und die Regeln für den Schlagwortkatalog (RSWK). In: Bibliotheksdienst, Jg. 38, H. 6, S. 745-791. Online verfügbar unter http://www.zlb.de/aktivitaeten/bd_neu/heftinhalte/heft9-1204/Erschliessung030604.pdf, zuletzt geprüft am 16.02.2008, hier S. 745 
aller Regel von Seiten der Wissenschaft die Forderung nach einer besseren formalen und vor allem inhaltlichen Erschließung der alten Bestände erhoben, was grundsätzlich berechtigt ist, da auf diesem Gebiet generell erhebliche Rückstände aufzuarbeiten sind." Er liefert auch gleich die Gründe für diese missliche Lage, denn „als Bibliothekare verweisen wir dann auf fehlende personelle und finanzielle Ressourcen, die entsprechende Unternehmungen verhindern. Wer nun - aufgrund der Kritik aus Forscherkreisen - Mut faßte und Drittmittel für die Sacherschließung gedruckter Bücher des Erscheinungszeitraums 1500 bis 1800 bei Förderinstitutionen erwerben wollte, müßte wohl die Erfahrung machen, daß sein Begehren nicht einmal die Hürde der Tagesordnung nehmen könnte, da es bereits im Vorfeld als zu teuer, da personalintensiv, und zu zeitaufwendig, da lediglich wenige Fremdleistungen zur Verfügung stehen, eingeschätzt werden würde. Sacherschließung alter Drucke in größerem Umfang, gleichgültig ob verbal oder systematisch, hat gegenwärtig keine - oder jedenfalls kaum eine Chance auf Förderung." ${ }^{15}$

Altbestände wurden in der Vergangenheit häufig als nutzlos betrachtet. In Zeiten von knappen öffentlichen Kassen und dem verstärkten Einzug wirtschaftlichen Denkens in die Bibliotheken besteht die Gefahr, dass die vorhandenen Altbestände lediglich als Ballast für die Bibliotheken angesehen werden. Dies hängt auch mit dem allgemeinen Siegeszug der Natur- und Ingenieurswissenschaften zusammen, die ihren Bedarf an wissenschaftlicher Literatur nicht mit Historischen Beständen befriedigen, im Gegenteil, diesen sogar eher als verzichtbar ansehen und der Rückdrängung der Geisteswissenschaften. Jedoch gibt es auch eine entgegengesetzte Entwicklung. Gerade die Geisteswissenschaften, die eigentliche Klientel für alte Drucke, sind sich der Bedeutung dieser alten Bestände durchaus bewusst. Sie fordern von den Bibliotheken, für eine bessere inhaltliche Auffindbarkeit und somit für bessere Arbeitsgrundlagen zu sorgen. Zudem wird das Alte Buch als Kulturgut auch für Nicht-Wissenschaftler zunehmend interessant. Zahlreiche gutbesuchte Ausstellungen, in denen die alten Werke in den Fokus der Öffentlichkeit gerückt werden, belegen dies. ${ }^{16}$ Mit der Nachfrage nach historischen Beständen steigt auch das Bedürfnis, diese möglichst schnell und effizient aufzufinden. Dies scheitert jedoch häufig an der mangelnden Erschließung, deren Durchführung wiederum an den oben genannten Gründen scheitert. ${ }^{17}$

15 Arnold, Werner (1997): Zur klassifikatorischen Sacherschließung alter Drucke, vornehmlich am Beispiel der Herzog August Bibliothek Wolfenbüttel. In: Bibliothek. Forschung und Praxis, Jg. 21, H. 3, S. 333-338. Online verfügbar unter http://www.bibliothek-saur.de/1997_3/333338.pdf, zuletzt geprüft am 16.02.2008, hier S. 333. Obwohl der Text bereits 1997 erschienen ist, existieren diese Probleme nach wie vor.

16 So waren z.B. 72.000 Besucher bei der in den Jahren 2003 und 2004 von der Badischen Landesbibliothek durchgeführten Ausstellung „Uns ist in alten Mären ... Das Nibelungenlied und seine Welt", bei der sowohl die Handschrift A, die Handschrift B sowie die Handschrift C des Nibelungenliedes ausgestellt wurden. Vgl. hierzu: Uns ist in alten Mären ... Das Nibelungenlied und seine Welt (2008). Badische Landesbibliothek. Online verfügbar unter http://www.blb-karlsruhe.de/blb/blbhtml/aktuelles/nibelungen.html, zuletzt aktualisiert am 02.01.2008, zuletzt geprüft am 24.02.2008.

17 Vgl. hierzu u.a. Fabian, Bernhard (2004): Brauchen wir ein Verzeichnis der deutschen Drucke des achtzehnten Jahrhunderts? In: Schnelling, Heiner (Hg.): VD 18. Verzeichnis der im deutschen Sprachraum erschienenen Drucke des 18. Jahrhunderts. Beiträge eines DFGRundgesprächs in der Universitäts- und Landesbibliothek Sachsen-Anhalt in Halle (Saale), 
Probleme bei der Altbestandserschließung sind u.a. darauf zurückzuführen, dass Werke des 16. und 17. Jahrhunderts häufig in lateinischer und französischer Sprache verfasst sind und die deutschsprachigen Drucke oft eine frühere, nicht normierte Form des Deutschen in Bezug auf Ortographie und Grammatik verwendeten. Ein Merkmal der Buch- und Druckproduktion des 18. Jahrhunderts hingegen sind die zahlreichen, bibliographisch schwer zu verzeichnenden Gattungen. Klein- und Kleinstdrucke, Gelegenheitsschriften, ephemeres und unkonventionelles Schriftum, in Zeit-, Ort- und Anlass gebunden (z.B. Theatertexte, im Selbstverlag publizierte Werke), Qualifikationsschriften (z.B. Schulschriften) und Akademieschriften spielen eine zunehmende Rolle. Dazu kommen zahlreiche, nur sehr aufwendig zu erschließende Sammelbände. Sind sie bereits erschlossen, dann oft nur unzureichend, d.h. meist nur der erste enthaltene Band. $^{18}$

All diese Umstände erschweren eine inhaltliche Erschließung von Historischen Beständen beträchtlich. Sie ist mit erheblich größerem Aufwand verbunden als die Sacherschließung von moderner Literatur. Es muss viel Zeit, Geld und Arbeitskraft in sie investiert werden.

veranstaltet am 05.05.2004. Halle (Saale): Univ.- und Landesbibliothek Sachsen-Anhalt (Schriften zum Bibliotheks- und Büchereiwesen in Sachsen-Anhalt, 86), S. 1-9

18 Vgl. Erste Planungen für ein Verzeichnis der im deutschen Sprachraum erschienenen Drucke des 18.Jahrhunderts (VD 18) (2004). In: Bibliotheksdienst, Jg. 38, H. 12, S. 1565-1571. Online verfügbar unter http://www.zlb.de/aktivitaeten/bd_neu/heftinhalte/heft9-1204/AltBuch1204.pdf, zuletzt geprüft am 16.02.2008, hier S. 1567 


\section{Die Erschließungssituation im Überblick}

\subsection{Die nationale Entwicklung seit 1979}

1979 erschien der erste Band des Gesamtverzeichnisses des deutschsprachigen Schrifttums (GV) 1700-1900, des GV-alt. ${ }^{19}$ Es verzeichnet sämtliche deutschsprachige Schriftenklassen sowie alle im deutschen Sprachraum erschienenen Drucke gleich welcher Sprache. Um die Daten für dieses Nachweiswerk zu gewinnen, „wurden 178 Buchhandels- und Fachbibliographien ${ }^{20}$ sowie gedruckte Kataloge reprographisch in ein Alphabet mit etwa zwei Millionen Titeln gebracht. Das Verzeichnis empfiehlt sich dem Benutzer also durch einen hohen Rationalisierungseffekt [...] schreibt aber bereits vorhandene Irrtümer, Fehler und Ungenauigkeiten weiter fort. Das GV-alt muss also mit genauso viel Vorsicht wie jedes der integrierten Verzeichnisse einzeln benutzt werden." ${ }^{21}$ Insgesamt beträgt der Umfang ca. 2 Mio. Bände. Die Titel wurden ausschließlich formal erschlossen. Auf eine sachliche Erschließung wurde verzichtet. Ebenso verzichtet wurde auf eine Übernahme der vorhandenen Sacherschließungsdaten der Quellen. In Band 1 findet neben der formalen eine sachliche Erschließung lediglich der ausgewerteten Bibliographien statt. ${ }^{22}$ Von 1976 bis 1981 kam noch das GV-neu hinzu, das Gesamtverzeichnis des deutschsprachigen Schrifttums (GV) 1911-1965.

Initiiert durch die Bayerische Staatsbibliothek München (BSB), die ihren alten Bandkatalog mit den Erwerbungen bis zum Bearbeitungsjahr 1840 in maschinenlesbare Form konvertieren wollte, legte die DFG 1979 ein Förderprogramm zur AltbestandserschlieBung ( $A B E)$ auf. Daraus ging der Aufbau eines kooperativen EDV-Katalogs als Nachweisinstrument für Altbestände hervor. Ziel war es, aus 15 Bibliotheken ${ }^{23}$ circa fünf Millionen Bestandsnachweise von alten Drucken aus den Jahren 1501 bis 1850 zu konvertieren. U.a. nahmen aus Bayern die Bayerische Staatsbibliothek, die Universitätsbibliothek der Ludwig-Maximilians-Universität München und die Staats- und Stadtbibliothek Augsburg teil, die ihre Kataloge in den Jahren 1983 bis 1998 konvertierten. ${ }^{24}$ Die Erschließung erfolgte dabei grundsätzlich durch Retrokonversion vorhandener Titelda-

19 Gesamtverzeichnis des deutschsprachigen Schrifttums (GV) 1700-1910 (1979-1987). Bearb. unter der Leitung von Peter Geils und Willi Gorzny. 160 Bände. München: Saur.

20 In ca. 560 Bänden. Vgl. Rösch, Hermann (2005): Lehrveranstaltung Allgemeine Informationsmittel. Begleitende Informationen. Erarbeitet unter Mitarbeit von Sonja Härkönen. Online verfügbar unter http://www.fbi.fh-koeln.de/institut/personen/roesch/Material_Roesch/Informationsmittel/Inhalt.htm\#inhalt7.3, zuletzt aktualisiert am 26.01.2005, zuletzt geprüft am 16.02.2008, hier Kap. 7: Nationalbibliographien

21 Hartwieg, Ursula (2004): Projekt "Verzeichnis der deutschen Drucke des 18.Jahrhunderts". In: Zeitschrift für Bibliothekswesen und Bibliographie, Jg. 51, H. 4, S. 222-228, hier S. 223

22 Vgl. Rösch, Hermann (2005), hier Kap. 7: Nationalbibliographien

23 Plus des Zentralkatalogs des Hochschulbibliothekszentrum Nordrhein-Westfalen (HBZ), der Nachweise von circa 70 weiteren nordrhein-westfälischen Bibliotheken enthielt

24 Holbach, Werner (2006): Retrokonversion und Retrokatalogisierung in den Bayerischen Staatlichen Bibliotheken. In: Bibliotheksforum Bayern, Jg. 34, H. 3, S. 206-228, hier S. 209 
ten anstatt per Autopsie, da der Aufwand dafür als zu groß empfunden wurde. ${ }^{25}$ Über die sachliche Erschließung in diesem Projekt äußert sich Syré 1987 in „Altbestandserfassung in wissenschaftlichen Bibliotheken der Bundesrepublik Deutschland ${ }^{\text {¿26 }}$ exemplarisch über die allgemeinen Probleme, die beim inhaltlichen Erschließen von Altbeständen auftreten:

„Wie die exemplarspezifische setzt die sachliche Erschließung das Vorliegen des Buches voraus. Sie setzt zweitens voraus, daß auch wissenschaftlich qualifizierte Mitarbeiter in ein ABE-Projekt eingebunden werden. Wie sehr sich die Bearbeitungszeit pro Titel verlängert, ist nur schwer abzuschätzen. [...] Altbestände bestehen [...] zu einem großen Teil aus „problematischen“ Literaturgattungen und Werken in lateinischer Sprache sowie deutschsprachigen Werken auf älterer Sprachstufe; die Lesbarkeit alter Schriften und Titelblätter kann ebenso Schwierigkeiten machen wie die Terminologie früherer Jahrhunderte, die Anwendung alter Klassifikationen oder die Übersetzung alter Begriffe in die heutige Sprache. Falls [...] für die sachliche Erschließung moderner Literatur 10 Minuten angesetzt werden, dann ist bei Altbeständen mit einer wesentlich längeren Bearbeitungszeit zu rechnen." Man „schätze daher, daß sich der [...] erforderliche Aufwand [...] quantitativ vermutlich nahezu verdoppeln dürtte. Neben den organisatorischen und personellen, in letzter Instanz natürlich finanziellen Problemen ist ein nicht minder schwieriges, bibliothekarisches Problem zu lösen: Nach welcher Methode sollen die Altbestände sachlich erschlossen werden? Die meisten Altbestände dürften systematisch aufgestellt sein, doch existieren wahrscheinlich fast ebensoviele Systematiken wie Bibliotheken; einige haben über ihren Entstehungsort hinaus Verbreitung gefunden (z.B. die Methode Eppelsheimer und das Hartwigsche System; Anm. d. Verf.). Daß man sich auf eine bestehende Klassifikation einigt oder daß eine neue, auf Altbestände zugeschnittene Klassifikation entworfen wird, muß wohl in den Bereich der Utopie verwiesen werden. Eine Systematisierung wäre in jedem Fall alter Literatur angemessener als eine Schlagwortvergabe, zumal es keine für alte Literatur brauchbare Methode der verbalen Sacherschließung gibt. Die Regeln für den Schlagwortkatalog (RSWK) sind für neuere Publikationen entwickelt worden und in ihrer Anwendung auf alte Titel nicht erprobt."

Aus diesen Punkten schließt er, „daß Sacherschließung, obwohl sie von Seiten der Wissenschaft gefordert wird und obwohl sie zu den originären Aufgaben der Bibliotheken zählt, auf überörtlicher Ebene im Rahmen der ABE nicht geleistet werden kann. Sacherschließung muß auf die lokale Ebene begrenzt bleiben, sofern sie nicht überhaupt Gegenstand der Fachbibliographie ist. Soweit im Rahmen der ABE über die Signatur Systemnotationen miterfasst werden, ergibt sich eine rudimentäre Sacherschließung, die für den lokalen Onlinekatalog nützlich sein kann, dem Benutzer der nationalen Altbestandsdatenbank aber kaum Vorteile bringt, da er nicht die Systematiken aller Teilnehmerbibliotheken kennen kann; er erführe lediglich etwas über die inhaltliche Zuordnung eines auf andere Weise gefundenes Werk (Freitextsuche). Eine gewisse sachliche Erschließung bieten die Stichwörter. Sacherschließungsmerkmale (Schlagwörter, Klassifikationsnummern) erhält man

25 Vgl. Hartwieg, Ursula (2004), hier S. 223

26 Syré, Ludger (1987): Altbestandserfassung in wissenschaftlichen Bibliotheken der Bundesrepublik Deutschland. Berlin: Dt. Bibliotheksinst. (DBI-Materialien, 67), hier S. 35-37 
auch durch die Übernahme amerikanischer Daten, doch stehen diese nur für einen kleinen Teil des gesamten deutschen Altbestands zur Verfügung. Sie lassen sich zur Herstellung von Stichwortkatalogen und zur Suche in der Datenbank verwenden. Die allgemein bekannte Unzulänglichkeiten der Stichwortmethode (Sprachgebundenheit, Synonyme, Orthographie usw.) würden jedoch bei Altbeständen, die zum großen Teil aus lateinischen Werken bestehen bzw. einer Zeit entstammen, als sich die deutsche Schriftsprache noch in der Entwicklung befand, besonders durchschlagen. Sinnvoll, aber aufwendig wäre es daher, die Stichwörter zu normieren bzw. in zusätzlichen Kategorien im Anschluß an die bibliographische Beschreibung in normierter Form aufzuführen; dazu wäre kein Autopsieverfahren notwendig, wohl jedoch ein Regelwerk bzw. ein kontrolliertes Vokabular."Er endet mit der Feststellung, dass „eine echte Sacherschließung [...] im Rahmen der ABE aus sachlichen und finanziellen Gründen - die Kosten würden sich wahrscheinlich verdoppeln - nicht leistbar"wäre.

Jedoch muss man bedenken, dass dieser hier zitierte Beitrag bereits aus dem Jahr 1987 stammt. Die angesprochenen Probleme mit der Terminologie früherer Jahrhunderte führen zwar nach wie vor zu Schwierigkeiten bei der sachlichen Altbestandserschließung. Darauf wird in Kap. 6.1.2. näher eingegangen. Dabei ist aber zu berücksichtigen, dass sich mit der fortschreitenden Entwicklung der EDV seit 1987 neue Möglichkeiten ergeben haben, historische Aufstellungssystematiken überregional zu nutzen, wie dies u.a. in Kap. 7.3. erläutert wird. Zuletzt kann man die Behauptung, dass es für alte Literatur keine brauchbare Methode der verbalen Sacherschließung gibt, nicht unkommentiert stehen lassen. So wird u.a. in Kap. 4.8. anhand dem Beispiel der Österreichischen Nationalbibliothek aufgezeigt, dass es durchaus sinnvoll sein kann, die Regeln für den Schlagwortkatalog (RSWK) auch auf alte Bestände anzuwenden.

Mit diesem Projekt der Altbestandserschließung durch Retrokonversion sollte erstmals das Fehlen einer deutschen Nationalbibliographie ausgeglichen werden, auch ging daraus die Personennamendatei (PND) hervor.

Das Handbuch der historischen Buchbestände erschien von 1991 bis 2001 in drei Abteilungen im Georg Olms Verlag. ${ }^{27}$ Angeregt wurde es erstmals 1983 von Bernhard Fabian. ${ }^{28}$ Es „,versteht sich als Inventar zu dem vom Beginn des Buchdrucks bis zum Ausgang des neunzehnten Jahrhunderts erschienenen Schrifttum, soweit dieses in allgemein zugänglichen Bibliotheken aufbewahrt wird. Zu historischen Beständen rechnen Bücher, Zeitschriften, Zeitungen, Musikdrucke, Karten und ephemeres Schrifttum.“29

27 Fabian, Bernhard (Hg.) (1992-2000): Handbuch der historischen Buchbestände in Deutschland. In Zusammenarbeit mit Severin Corsten ... Hrsg. von Bernhard Fabian. 27 Bände in 28 Bänden. Hildesheim: Olms-Weidmann.

Seit dem 8. Mai 2007 ist das Handbuch auch online abrufbar unter: b2i, Wissenschaftsportal (2008): Fabian-Handbuch: Home. Online verfügbar unter http://www.b2i.de/fabian, zuletzt geprüft am 16.02.2008.

28 Vgl. dazu Fabian, Bernhard (1983): Buch, Bibliothek und geisteswissenschaftliche Forschung. Zu Problemen der Literaturversorgung und der Literaturproduktion in der Bundesrepublik Deutschland. Göttingen: Vandenhoeck \& Ruprecht (Schriftenreihe der Stiftung Volkswagenwerk, 24).

29 Münster, Westfälische Wilhelms-Universität; Institut für Buchwissenschaft und Textforschung (2007): IBT - Handbuch der historischen Buchbestände. Westfälische Wilhelms-Universität 
Handschriften wurden nicht verzeichnet. Man begriff das Handbuch also als eine Art Dokumentation der vorhandenen Bestände und als Orientierungsquelle für die Geistesund Kulturwissenschaften. Zudem sollte es als Hilfsinstrument in der täglichen bibliothekarischen Praxis eine Ergänzung zu den bereits vorhandenen Katalogen darstellen. Jede beteiligte Bibliothek fügte einen Beitrag bei, in dem neben administrativen Informationen über die Bibliothek, Angaben zur Bestandsgeschichte, Angaben über die vorhandenen Kataloge und weiteren Punkten auch eine ausführliche Beschreibung des jeweiligen Altbestandes plus eventuell vorhandener Sondersammlungen verzeichnet war. In dieser Beschreibung sollten sowohl die zeitliche, die sprachliche als auch die fachliche Zusammenstellung ausführlich erläutert werden. Diese sollte möglichst genau und in kleinen Bestandsgruppen unterteilt ausfallen, da man so tief wie möglich fachlich unterscheiden und, damit verbunden, sachlich erschließen wollte. ${ }^{30}$ Jedoch war dies die genaueste inhaltliche Erschließung. Einzelne Titel wurden nicht erschlossen, höchstens thematisch ähnliche Werke im Ganzen. Diese Erschließung folgte natürlich keinen bestimmten Regeln und keiner Systematik, da ja jede Bibliothek für sich ihren Bestand erläuterte, sondern lediglich in frei beschreibender, oft nur sehr grober Form.

Die kurze Bestandsbeschreibung einer sehr kleinen Spezialibliothek sieht z.B. folgendermaßen aus:

Bestandsbeschreibung der Bibliothek des Forschungsinstituts für Vor- und Frühgeschichte Unteruhldingen:

„Vom heutigen Gesamtbestand von rund 15.000 Titeln entfallen 576 Titel auf den Zeitraum bis 1900. Die meisten historischen Titel (550) sind im 19. Jh. erschienen. Das 17. Jh. weist einen Titel, das 18. Jh. 25 auf. Weitere 1700 Titel entfallen auf den Zeitraum 1901 bis 1920. Für die Jahre 1920 bis 1946 wurden 5000 Titel gezählt. Die Aufstellung ist innerhalb von regionalen oder thematischen Gruppen alphabetisch nach Autoren.

Den Hauptbestandteil bildet das Sammelgebiet der Vor- und Frühgeschichtlichen Archäologie und ihrer zahlreichen Nachbarwissenschaften. Gut vorhanden sind die Fachzeitschriften Deutschlands und der Schweiz, ebenso Standard- und Nachschlagewerke zur Vor- und Frühgeschichte. Die Pfahlbauliteratur ist so gut wie vollständig vertreten. Aus der Zeit vor 1945 sind zahlreiche Werke zur Vor- und Frühgeschichte Ostdeutschlands und des ehemaligen Ost- und Westpreußen vorhanden. Ständig erweitert werden die Bestände zu den Themenbereichen Technik/Experimentelle Archäologie sowie Museumspädagogik. Außerdem sind die Bereiche Anthropologie, Biologie, Ethnologie, Geowissenschaften, Geschichte, Philologie, Religionsforschung und Volkskunde repräsentiert. Einen weiteren Bereich stellt Heimatliteratur des Bodenseeraumes dar. Besonders erwähnenswert ist

Münster. Online verfügbar unter https://www.uni-muenster.de/Buchwiss/Forschen/Handbuch_der_historischen_Buchbestaende.html, zuletzt aktualisiert am 07.11.2007, zuletzt geprüft am 16.02.2008.

30 Vgl. u.a. Corsten, Sven (1985): Das Projekt zur Erschließung der historischen Buchbestände in Deutschland. In: Frankenberger, Rudolf; Habermann, Alexandra (Hg.): Literaturversorgung in den Geisteswissenschaften. 75. Deutscher Bibliothekartag in Trier 1985. Frankfurt am Main: Klostermann (Zeitschrift für Bibliothekswesen und Bibliographie : Sonderhefte, 43), S. 186-193. 
die sehr umfangreiche Sammlung völkisch-rassischer Literatur zum Thema Vorund Frühgeschichte. ${ }^{‘ 31}$

Die Arbeitsgemeinschaft Sammlung Deutscher Drucke (AG SDD) ${ }^{32}$ ist ein Zusammenschluss sechs deutscher Bibliotheken mit dem Ziel der Bildung einer virtuellen Nationalbibliothek. Dabei ist jede Bibliothek für ein bestimmtes Zeitsegment verantwortlich. Die Idee zu dieser Kollaboration hatte ebenso wie für das Handbuch der historischen Buchbestände, Bernhard Fabian. ${ }^{33}$ Seit 1989 existiert diese Arbeitsgemeinschaft; die Deutsche Nationalbibliothek ist seit 1995 beteiligt. Ausgewählt wurden die jeweiligen Bibliotheken, da sie für ihr spezifisches Zeitsegment bereits über einen großen Bestand verfügen, der nun mittels retrospektiver Erwerbung sukzessive ausgebaut wird. Erworben werden alle im deutschen Sprachgebiet erschienenen Drucke unabhängig der Sprache, sowie alle außerhalb des deutschen Sprachraums gedruckten Werke in deutscher Sprache.

Folgende Bibliotheken sind an der Kooperation beteiligt, dargestellt mit dem jeweiligen Zeitfragment:

\begin{tabular}{|l|l|}
\hline $1450-1600$ & Bayerische Staatsbibliothek München \\
\hline $1601-1700$ & Herzog August Bibliothek Wolfenbüttel \\
\hline $1701-1800$ & Staats- und Universitätsbibliothek Göttingen \\
\hline $1801-1870$ & Universitätsbibliothek J. C. Senckenberg, Frankfurt am Main \\
\hline $1871-1912$ & Staatsbibliothek zu Berlin \\
\hline 1923 ff. & Deutsche Nationalbibliothek \\
\hline
\end{tabular}

Die inhaltliche Erschließung erfolgt für die einzelnen Zeitabschnitte unterschiedlich. So werden die Drucke des 17. Jahrhunderts mit Gattungsbegriffen erschlossen, ${ }^{34}$ die Drucke des 18. Jahrhunderts ebenfalls, hier jedoch zusätzlich mit der Basisklassifikation (vgl. Kap. 4.3.).

Die Drucke aus dem Zeitsegment 1801-1870 hingegen bekommen lediglich einen Fachcode, der jedoch nur im lokalen OPAC recherchierbar ist. In einigen Fächern er-

31 Fabian, Bernhard (Hg.) (1992-2000): Band 9: Baden-Württemberg und Saarland. T-Z, hier S. 165.

32 AG-SDD, AG Sammlung Deutsche Drucke - Home (2007). Online verfügbar unter http://www.ag-sdd.de/, zuletzt aktualisiert am 11.04.2007, zuletzt geprüft am 16.02.2008.

33 Vgl. dazu Fabian, Bernhard (1983)

34 Peitz, Peter (1997): Erschließung älterer Drucke. Was ist nötig, was ist möglich? In: ProLibris, Jg. 2, H. 2, S. 99-103, hier S. 101. Arnold hingegen schreibt: „In dem Projekt „Sammlung Deutscher Drucke“ werden bei der Katalogisierung der Erwerbungen des 17. und 18. Jahrhundert die Basisklassifikation und die Schlagwortnormdatei (SWD) als SacherschlieBungsinstrumente eingesetzt." Vgl. Arnold, Werner (1997), hier S. 228 
folgt eine zusätzliche Erschließung mit RSWK ${ }^{35}$ Die Staatsbibliothek Berlin erschließt alle neu erworbenen Drucke klassifikatorisch nach der Systematik ihres Alten Realkatalogs ${ }^{36}$ worauf in Kap. 4.4. näher eingegangen wird.

35 Diese Angaben stammen aus einer E-Mail von Frau Dr. Angela Hausinger, Ansprechpartnerin für das Zeitsegment 1801 - 1870 der SDD in der UB Frankfurt, vom 15.05.2007

36 Vgl. AG-SDD, Staatsbibliothek zu Berlin - Preußischer Kulturbesitz (2007). Online verfügbar unter http://www.ag-sdd.de/bibliotheken/spk.htm, zuletzt aktualisiert am 21.02.2007, zuletzt geprüft am 16.02.2008. 


\subsection{Die Retrospektive Nationalbibliographie}

Aufgrund der speziellen geschichtlichen Entwicklung Deutschlands, geprägt von Kleinstaaterei und Föderalismus, existiert in Deutschland im Gegensatz zu anderen Ländern keine gewachsene Nationalbibliographie. Diese Nationalbibliographien erwuchsen in Ländern wie den USA (Library of Congress), Großbritannien (British Library) oder Frankreich (Bibliothèque nationale de france) aus dem Sammelauftrag einer zentralen Institution. ${ }^{37}$ In Deutschland existiert dieser Sammelauftrag erst seit 1913, seit der Gründung der Deutschen Bücherei in Leipzig.

Um dieses Fehlen einer Nationalbibliographie auszugleichen, startete man 1969 mit einem Projekt, welches die Verzeichnung der Drucke des 16. Jahrhunderts zum Ziel hatte. 1980 begannen die Planungen für das 17. Jahrhundert und seit 2004 werden die Vorraussetzungen und Möglichkeiten für ein Verzeichnis des 18. Jahrhunderts geprüft.

\subsubsection{Das VD 16}

Mit der Errichtung je einer Arbeitsstelle in der Bayerischen Staatsbibliothek München und in der Herzog August Bibliothek in Wolfenbüttel wurde 1969 das von der Deutschen Forschungsgemeinschaft (DFG) geförderte Verzeichnis der im deutschen Sprachbereich erschienenen Drucke des 16. Jahrhundert (VD 16) ins Leben gerufen. Das VD 16 versteht sich als deutsche Nationalbibliographie des 16. Jahrhunderts und verzeichnet momentan ${ }^{38}$ rund 100.000 Titel. $^{39}$

Erschlossen werden die im deutschen Sprachgebiet gedruckten Werke des 16. Jahrhunderts, unabhängig ihrer Sprache. Dies geschieht mittels autoptischer retrospektiver Katalogisierung; typographische Besonderheiten (z.B. Abbreviaturen) werden bei der formalen Erschließung beibehalten. ${ }^{40}$ Das in der Printausgabe 22 Bände umfassende Werk wurde 2004 retrokonvertiert. ${ }^{41}$

Eine inhaltliche Erschließung der Drucke entfällt ganz, sie findet weder verbal noch klassifikatorisch noch in sonstiger Weise statt und ist auch für die Zukunft nicht angedacht. Deswegen soll das VD 16 im Rahmen dieser Arbeit auch nicht weiter betrachtet werden.

37 Stäcker, Thomas (2004): VD 17 - mehr als eine Zwischenbilanz. In: Zeitschrift für Bibliothekswesen und Bibliographie, Jg. 51, H. 4, S. 213-221, hier S. 213

38 Stand: 16.02 .2008

39 Verzeichnis des deutschsprachigen Schrifttums des 16. Jahrhunderts (2008). Online verfügbar unter http://www.vd16.de/, zuletzt geprüft am 16.02.2008.

40 Vgl. Möncke, Gisela (2004): Das "Verzeichnis der im deutschen Sprachbereich erschienenen Drucke des 16.Jahrhunderts" (VD 16) als Teil einer deutschen retrospektiven Nationalbibliographie. In: Zeitschrift für Bibliothekswesen und Bibliographie, Jg. 51, H. 4, S. 207-212, S. 207

41 Vgl. Holbach, Werner (2006), hier S. 212 


\subsubsection{Das VD 17}

Im Verzeichnis der im deutschen Sprachraum erschienenen Drucke des 17. Jahrhunderts (VD 17) werden alle deutschsprachigen sowie alle im deutschen Sprachgebiet gedruckten Werke gleich welcher Sprache verzeichnet. Beginn der auf Autopsie beruhenden Katalogisierung war der 01.07.1996. Die von der DFG geplante Förderzeit wurde auf 10-12 Jahre veranschlagt. Das VD 17 soll jedoch auch nach Ablauf der Förderungszeit, die momentan noch läuft, weitergeführt und ergänzt werden.

Ziel des VD 17 ist die Schaffung einer retrospektiven Nationalbibliographie der Jahre 1601-1700. Momentan enthält die Datenbank 246.680 Titel mit 457.750 Nachweisen. ${ }^{42}$

Im Gegensatz zum VD 16 wurde das VD 17 von Beginn an als elektronische Datenbank konzipiert. Dadurch war es möglich, den Titeldaten digitale Images bestimmter Schlüsselseiten (Titelseite, Kolophon etc.) beizugeben. Durch diese Images sind die einzelnen Drucke schnell und sicher identifizierbar. Dadurch ist es nicht mehr nötig, die Titelaufnahmen typographisch genau, inklusive aller Besonderheiten aufzunehmen, so wie dies noch im VD 16 gehandhabt wurde.

Eine weitere bibliographische Neuerung ist die kontinuierliche Anwendung des Fingerprints. ${ }^{43}$ Dieser besteht im Kern aus einer Zeichenkombination von 16 Zeichen, eingeteilt in vier Gruppen, die dem jeweiligen Druck auf verschiedenen Seiten entnommen werden. Komplettiert werden diese Zeichen mit einem Indikator, dem Erscheinungsjahr des Werkes und einem weiteren Indikator. Diese Zeichenkombination dient der Zusammenführung gleicher Ausgaben sowie der Unterscheidung von Drucken unterschiedlicher Ausgaben ohne physischen Vergleich. ${ }^{44}$

Auch Müller weist wieder in seiner 1990 erschienenen Vorstudie „Die Drucke des 17. Jahrhunderts im deutschen Sprachraum. Untersuchungen zu ihrer Verzeichnung in einem VD 17. ${ }^{45}$ auf den Wunsch der Wissenschaft nach einer Sacherschließung der im VD 17 zu verzeichnenden Drucke hin:

"Eine sachliche Erschließung auch der alten Drucke wird zweifellos von der Wissenschaft dringend gewünscht und benötigt, wie die tägliche bibliothekarische Praxis beweist; denn ohne Sachzugang zur Literatur kann eigentlich immer wieder nur das Bekannte, das heißt das nach Autor bzw. Sachtitel bereits zitierte Werk, gesucht und gefunden werden. Einen wesentlichen Fortschritt in der Erforschung der Quellen des 17. Jahrhunderts durch deren Verzeichnung würde daher erst eine zusätzliche inhaltliche Erschließung erbringen."

42 Verzeichnis der Drucke des 17. Jahrhunderts (2007). Online verfügbar unter http://www.vd17.de/, zuletzt aktualisiert am 11.04.2007, zuletzt geprüft am 16.02.2008.

43 Vgl. Stäcker (2004), hier S. 214

44 Haller, Klaus (Hg.) (1994): Regeln für die Katalogisierung alter Drucke. Erarbeitet von der Arbeitsgruppe des Deutschen Bibliotheksinstituts "RAK-WB und Alte Drucke". Herausgegeben und eingeleitet von Klaus Haller. Berlin: Dt. Bibliotheksinst. (Schriften der Deutschen Forschungsgemeinschaft), hier S. 38

45 Müller, Wolfgang (1990): Die Drucke des 17. Jahrhunderts im deutschen Sprachraum. Untersuchungen zu ihrer Verzeichnung in einem VD17. Im Auftrag der Deutschen Forschungsgemeinschaft vorgelegt von Wolfgang Müller. Wiesbaden: Harrassowitz (Beiträge zum Buchund Bibliothekswesen, 31). 
Jedoch räumt auch er Probleme bei der sachlichen Erschließung von Drucken des 17. Jahrhunderts ein:

„Leider gibt es unter den existierenden nationalbibliographischen Unternehmen keine bedarfsgerechten Vorbilder. Eine überregionale Konzeption auf dem Gebiet der Sacherschließung von Altbeständen existiert ebenfalls nicht. [...] Generell würde eine Sacherschließung von Drucken des 17. Jahrhunderts fachliche Probleme aufwerfen, da sich in sehr vielen Sparten erst danach jene wissenschaftliche Terminologie gebildet hat, deren Elemente in den alten Werken noch ungenau oder mit abweichendem Sinn gebraucht werden. [...] Sacherschließung setzt in jedem Falle Autopsie voraus" und "erfordert darüber hinaus nicht nur den Einsatz eines größeren, sondern eines qualifizierteren Mitarbeiterstabes, der alle Wissensbereiche und Sprachen abdeckt und in den Verhältnissen des 17. Jahrhunderts bewandert ist. ${ }^{\text {"46 }}$

So findet wie schon im VD 16 auch im VD 17 keine Sacherschließung statt. Als Gründe werden der hohe Zeitanspruch sowie mangelnde finanzielle Ressourcen angegeben. Zur Begründung zieht man somit also ebenfalls diejenigen Ursachen heran, die meistens in diesem Zusammenhang genannt werden. Damit jedoch ein, wenn auch nur grober inhaltlicher Sucheinstieg auf die einzelnen Drucke möglich ist, entschied man sich, sogenannte Gattungsbegriffe zu vergeben. Diese sind relativ einfach und schnell zugeteilt.

Dabei handelt es sich um rund 140 Begriffe und Fachgruppenbezeichnungen, die in einer Liste ${ }^{47}$ festgelegt und normiert sind. Als Grundlage für die Liste des VD 17 diente eine bereits erprobte Liste der Herzog August Bibliothek Wolfenbüttel. Diese wurde entsprechend den spezifischen Anforderungen einer Bibliographie des 17. Jahrhunderts modifiziert. ${ }^{48}$ Als Begriffe werden in dieser Liste sowohl Bezeichnungen ganzer Fachgebiete (z.B. Chemie, Geographie, Zoologie) als auch Bezeichnungen kleinerer Gruppen (z.B. Akustik, Chiromantie, Magnetismus), plus zahlreiche Formschlagwörter (z.B. Atlas, Auktionskatalog, Vorlesungsverzeichnis) geführt. ${ }^{49}$

Allerdings wird es bei der Überführung dieser Gattungsbegriffe in einen Katalog, in dem Schlagwortvergabe nach den RSWK angewandt wird, nötig, diese Daten auch in Schlagwortketten umzusetzen.

46 Vgl. Müller (1990), hier S. 68 f.

47 Verzeichnis der Drucke des 17. Jahrhunderts: Gattungsbegriffe (2007). Online verfügbar unter http://www.vd17.de/gattungsbegriffe.html, zuletzt aktualisiert am 11.04.2007, zuletzt geprüft am 16.02.2008.

48 Vgl. Müller (1990), hier S. 75

49 Vgl. Hoinkis, Gudrun (1999): Bisherige Erschließung der Drucke des 17. Jahrhunderts (VD17). In: Bibliotheksdienst, Jg. 33, H. 11, S. 1874-1885. Online verfügbar unter http://bibliotheksdienst.zlb.de/1999/1999_11_Erschlie02.pdf, zuletzt geprüft am 16.02.2008, S. 1883 
Dies kann z.B. folgendermaßen aussehen:

s Auktionskatalog ; z Geschichte ; f Quelle ${ }^{50}$

Vergeben werden Gattungsbegriffe indes nicht bei allen Drucken. Zum einen existieren nicht für jeden Druck passende Begriffe, ${ }^{51}$ zum anderen werden nur für jene Drucke Begriffe vergeben, bei denen diese leicht zu ermitteln sind. Bei Bedarf werden einem Druck auch mehrere Begriffe zugeteilt. Ein weiteres Problem stellt die geringe Trennschärfe bestimmter Begriffe dar (z.B. Alchemie und Chemie) ${ }^{52}$

Als Beispiel für einen Kleindruck ohne zugeordneten Gattungsbegriff folgt hier die Auflistung und Beschreibung einer Räuberbande aus dem 17. Jahrhundert:

\section{VD17 12:130537U}

Titel:

$$
\begin{aligned}
& \text { Verzeichnuß und Description Derjenigen Complicum, } \\
& \text { und andern Lands-verschraiten Jauner/ als Dieb und } \\
& \text { Rauber/ sambt dergleichen Weibsbildern/ welche bey } \\
& \text { dem Chur-Pfältz-Neuburgischen Pfleg-Ambt Heydeck } \\
& \text { in Verhafft gesessenen/ und unlängsten neben zweyen } \\
& \text { andern Nahmens Hanß Wörl und Lorentz Fischeysen } \\
& \text { mit dem Strang hingerichteten Andreas Schoneckher/ } \\
& \text { wie auch von andern angezeiget/ und nach ihrer Ge- } \\
& \text { stalt beschriben/ und zum Churfl. Hof-Rath München } \\
& \text { communicirt worden }
\end{aligned}
$$

Erschie[S.I.], [vor 1700]

nen:

Kollation:

[4] BI. ; ${ }^{\circ}$

Fingerprint:

n.zu u-e- er7. i-i- $C$

Sprache(n):

ger

Exemplar:

<12> München, Bayerische Staatsbibliothek

Signatur: 2 Bavar. 1400, VII,28

50 RSWK (1998): Regeln für den Schlagwortkatalog. 3., überarb. und erw. Aufl. Berlin: Dt. Bibliotheksinst. Anlage 10. Konkordanzen zu den Gattungsbegriffen des VD 17, hier S. A75. Online verfügbar unter http://deposit.ddb.de/ep/netpub/89/96/96/967969689/_data_stat/ www.dbi-berlin.de/dbi_pub/einzelpu/regelw/rswk/rswk_00.htm, zuletzt geprüft am 16.02.2008.

51 Verzeichnis der Drucke des 17. Jahrhunderts: Bibliographische Beschreibung (2007). Online verfügbar unter http://www.vd17.de/bibliographB.html, zuletzt aktualisiert am 24.04.2007, zuletzt geprüft am 16.02.2008.

52 Vgl. Stäcker (2004), hier S. 217 f. 
Die Arbeitsgruppe des Deutschen Bibliotheksinstituts „RAK-WB und Alte Drucke“ empfahl 1994 die Vergabe von Gattungsbegriffen bei historischen Beständen. Durch die Verwendung kann man „häufige Fragestellungen bei Benutzern abdecken, deren Forschungsgebiet spezielle Gattungen sind, z.B. Flugschriften des 16. Jahrhunderts." Sie können „hilfreich sein, wenn die Zuordnung eines Titels zu einem Verfasser oder/und die Titelfassung unklar ist.“ Des Weiteren können Gattungsbegriffe „ein erster Schritt in die sachliche Suche sein“ und sie sind „hilfreich bei der Online-Suche, um große Treffermengen einzuschränken." ${ }^{53}$

Obwohl sich mit Gattungsbegriffen nur grob selektieren lässt und man bei der einfachen Suche nach bestimmten Gattungen und Fachgruppen trotz der eigentlichen Einschränkung z.T. sehr große Treffermengen erhält, ist es durchaus möglich, durch die Anwendung bestimmter Suchkombinationen brauchbare und überschaubare Ergebnisse zu erhalten. Durch die schnelle und einfache Vergabe der Begriffe stehen Kosten und Aufwand der Anwendung einerseits sowie Nutzen der Anwendung für die Wissenschaft andererseits durchaus in einem günstigen Verhältnis.

Trotzdem kommt Müller 1990 zu dem Ergebnis: „Die Frage, ob systematische ErschlieBung und Erschließung nach Gattungen sich gegenseitig ersetzen können, muß alles in allem negativ beantwortet werden. ${ }^{\text {"55 }}$

\subsubsection{Das VD 18}

Ein VD 18 existiert im Gegensatz zu einem VD 16 und einem VD 17 noch nicht; es befindet sich erst in der Planung. 2004 fand ein Rundgespräch in der Universitäts- und Landesbibliothek Sachsen-Anhalt statt. Erörtert wurden dabei zentrale Fragen bezüglich des Bedarfs, der Notwendigkeit, den Vorraussetzungen, des Umfangs und den Möglichkeiten einer Umsetzung eines VD $18 .^{55}$ Da sich die Situation des Nachweises und der Erschließung des Schrifttums des 18. Jahrhunderts in Deutschland im internationalen Vergleich ${ }^{56}$ defizitär darstellt, wird seitens der Bibliotheken und vor allem sei-

53 Haller (1994), hier S. 43

54 Müller (1990), hier S. 75

55 Die einzelnen Beiträge sowie das Ergebnissprotokoll der Abschlussdiskussion sind verzeichnet in: Schnelling, Heiner (Hg.) (2004): VD 18. Verzeichnis der im deutschen Sprachraum erschienenen Drucke des 18. Jahrhunderts. Beiträge eines DFG-Rundgesprächs in der Universitäts- und Landesbibliothek Sachsen-Anhalt in Halle (Saale), veranstaltet am 05.05.2004. Halle (Saale): Univ.- und Landesbibliothek Sachsen-Anhalt (Schriften zum Bibliotheks- und Büchereiwesen in Sachsen-Anhalt, 86). Vgl. zu den Vorraussetzungen und Umsetzungsmöglichkeiten eines VD 18 auch Bötte, Gerd-Josef (2005): Das deutsche Schrifttum des achtzehnten Jahrhunderts und seine bibliographische Verzeichnung - Perspektiven für ein VD18. Online verfügbar unter http://www.opus-bayern.de/bibinfo/volltexte/2006/141/pdf/Boette-VD18_B-Tag2005-opusMS_pdf.pdf, zuletzt geprüft am 16.02.2008 sowie die bereits zuvor in anderem Zusammenhang zitierten Erste Planungen für ein Verzeichnis der im deutschen Sprachraum erschienenen Drucke des 18.Jahrhunderts (VD 18) (2004) und Hartwieg, Ursula (2004)

56 Vgl. dazu die Beispiele aus dem Ausland unter Kap. 3.3. 
tens der Wissenschaft gefordert, ${ }^{57}$ aufgrund der guten Erfahrungen mit dem VD 16 und dem VD 17 nun auch das 18. Jahrhundert in Angriff zu nehmen.

Jedoch sind die Voraussetzungen für eine Umsetzung des Projekts komplizierter als sie z.B. beim VD 17 waren. So steigt die Anzahl der Drucke im 18. Jahrhundert exorbitant an. Jefcoate rechnet mit 600.000 Einheiten, ${ }^{58}$ andererseits hält u.a. Bötte aufgrund der hohen Zahl von Klein- und Gelegenheitsschrifttum auch 750.000 Drucke für möglich. ${ }^{59}$ Die große Anzahl an bibliographisch schwierig zu verzeichnenden Gattungen stellt eine weitere Erschwernis dar, da geklärt werden muss, welche Materialien nachgewiesen werden sollen. Dabei wird gewünscht, dass sich ein VD 18 den Selektionskriterien der Vorgängerprojekte VD 16 und VD 17 anschließt, was bedeutet, dass eine Maximalkonzeption angestrebt werden soll. Diese beinhaltet sowohl alle Drucke aus den für diesen Zeitraum maßgebenden geographischen Grenzen als auch alle Drucke aus diesem Zeitraum, die in deutscher Sprache verfasst wurden, unabhängig vom Druckort. Erschlossen werden soll nach Autopsie, um die Qualität der bibliographischen Beschreibung zu gewährleisten. Neben anderen Punkten ist die Frage strittig, ob die Ermittlung von Kollationsformel oder Fingerprint erforderlich ist und inwieweit typographische Besonderheiten wiedergegeben sollen. Strittig ist ebenfalls, ob es möglich sein wird, wie von der Forschung gefordert unselbständige Werke zu verzeichnen. ${ }^{60}$ Ein weiterer wichtiger Punkt ist die Einbindung von Digitalisaten. So plädiert z.B. Bötte aufgrund der heutigen technischen Möglichkeiten für einen Ausbau der Digitalisierung. ${ }^{61}$ Im Gegensatz zum VD 17 sollte sie sich dabei nicht nur auf Schlüsselseiten ${ }^{62}$ beschränken, sondern man sollte Dokumente möglichst komplett digitalisieren, um direkt vom Katalognachweis darauf zugreifen zu können und „idealerweise jenseits der bibliographischen Ebene in den Tiefenstrukturen des digitalen Textkorpus recherchieren zu können.“63 „Die Erzeugung digitaler Volltexte mittels OCR-Software oder per manueller Dateneingabe wäre für die größtmögliche Anzahl von VD 18-Nachweisen höchst wünschenswert, weil gerade in diesem Bereich durch erweiterte Suchoptionen und Retrievalmöglichkeiten gegenüber dem bloßen bibliographischen Nachweis auf Ti-

57 Vgl. zu dieser Thematik auch die beiden Aufsätze: Siegert, Reinhart (2004): Defizite bibliothekarischer Kataloge aus Sicht der Forschung. In: Schnelling, Heiner (Hg.) (2004), S. 11-28 und Braungart, Georg (2004): Kanon und Forschung: die Bedeutung der Erschließung für die Literaturwissenschaft. In: Schnelling, Heiner (Hg.) (2004), S. 43-48

58 Jefcoate, Graham (2004): Zur quantitativen Präzisierung des Volumens der Literatur des 18. Jahrhunderts - Probleme und Lösungen im deutschsprachigen Raum und internationalen Bereich. In: Schnelling, Heiner (Hg.) (2004), S. 61-74, hier S. 70

59 Bötte, Gerd-Josef (2005), hier S. 4. Dies ist also zwei- bis dreimal soviel wie beim VD 17, bei dem man von einem Gesamtvolumen von 265.000 Einheiten ausgeht. Vgl. zu der Schätzmenge über die quantitative Buchproduktion im 17. Jahrhundert: Wittmann, Reinhard (1991): Geschichte des deutschen Buchhandels. Ein Überblick. München: Beck, hier S. 75 f.

60 Vgl. Bötte, Gerd-Josef (2005), hier S. 5

61 Bötte, Gerd-Josef (2004): VD 18: Vom bibliographischen Nachweis zum Digitalen Dokument. In: Schnelling, Heiner (Hg.) (2004), S. 95-109.

62 Unter Schlüsselseiten werden die Titelseite, Seiten mit Widmungsempfängern, die erste Seite des Haupteils, das Kolophon und Seiten mit Druckermarken und Verlagssignets verstanden.

63 Bötte, Gerd-Josef (2004), hier S. 95 
telebene der größte Mehrwert für den wissenschaftlichen Nutzer entsteht. "64 Dies schließt die Erzeugung hochwertiger struktureller Metadaten mit ein. Die Nachteile einer solchen aufwändigen Digitalisierung sind natürlich die hohen Kosten, die sie mit sich bringt. Jedoch besteht darin eine große Chance für eine bessere inhaltliche Erschließung und Auffindbarkeit, und zwar u.a. durch Recherchemöglichkeiten in den Volltexten und durch die Anwendung von maschinellen Indexierungsverfahren (Vgl. Kap. 7.5.). Diese Überlegungen leiten über auf die Frage nach der Sacherschließung; bezüglich der Thematik der vorliegenden Arbeit wohl die wichtigste im Kontext der Planung eines VD 18. Wie bereits in Kap. 3.1. angesprochen, erschließt die im Rahmen der AG Sammlung Deutscher Drucke für das 18. Jahrhundert zuständige Bibliothek, die SUB Göttingen, die Drucke sowohl per Gattungsbegriff als auch per Basisklassifikation (Vgl. auch Kap. 4.3.). Bei der Anwendung der Gattungsbegriffe bezieht sich die SUB Göttingen dabei auf die „Göttinger"-Liste, die dieselben Begriffe verwendet wie die Liste der Herzog August Bibliothek in Wolfenbüttel. Bei der Überarbeitung dieses Indexes für die Anwendung innerhalb der Sammlung Deutscher Drucke wurde auf Übereinstimmung mit der Formschlagwortliste der RSWK geachtet. Eine Verschlagwortung nach der Schlagwortnormdatei (SWD) wird dabei aufgrund der bereits angesprochenen terminologischen Schwierigkeiten nur sehr eingeschränkt vorgenommen. ${ }^{65}$ Es muss geprüft werden, ob die Vergabe von Gattungsbegriffen auch im VD 18 angewendet werden kann, und wenn ja, ob man dafür eine bereits bestehende Liste übernehmen kann oder ob man einen neuen, modifizierten Index von Gattungsbegriffen erstellen muss. Ebenfalls ist unklar, ob die Klassifizierung nach der Basisklassifikation übernommen werden soll. Auf eine weitere Möglichkeit der Sacherschließung weisst Thomas Bürger hin. ${ }^{66}$ Er plädiert für die Nutzung sprechender Signaturen historischer, sachlichsystematischer Aufstellungsordnungen alter Bibliothekskataloge ${ }^{67}$

\subsection{Die Situation im Ausland}

Im Gegensatz zu Deutschland ist die inhaltliche Erschließung von Historischen Bibliotheksbeständen in anderen Ländern teilweise bereits weit fortgeschritten, in einigen europäischen und nordamerikanischen Ländern ist sie Standard.

Die meisten Länder Europas und Nordamerikas haben infolge ihrer konträren Entwicklung im Gegensatz zu Deutschland eine zentrale, nationale Publikationen sammelnde Institution und somit eine über die vergangenen Jahrhunderte stetig gewachsene Nationalbibliographie. Dank dieser Konstellation befindet sich der historische Bestand u.a. zentral an einem Ort. Aus diesem Grund fällt eine Erschließung nach einheitlichen

64 Bötte, Gerd-Josef (2004), hier S. 102

65 Vgl. Hartwieg, Ursula (2004), hier S. 224

66 Bürger, Thomas (2004): Historische Drucke in Virtuellen Fachbibliotheken? Nutzen und Möglichkeiten sachlicher Sucheinstiege in das VD17 und VD18. In: Schnelling, Heiner (Hg.) (2004), S. 85-94.

67 Vgl. dazu die Ausführungen in Kap. 7.3. 
Standards wesentlich leichter als in Deutschland mit seiner föderalistischen Struktur und den zahlreichen verschiedenen Bibliotheksverbünden.

Eine Bezeichnung, die man im Zusammenhang mit Altbestandserschließung im Ausland sehr häufig für einen Katalog hört, der zentral die historischen Bestände für einen bestimmten Zeitraum eines bestimmten Landes oder Gebietes verzeichnet, ist „Short Title Catalogue“.

So gibt es z.B. den Short Title Catalogue Netherlands (STCN), ${ }^{68}$ die retrospektive Nationalbibliographie der Niederlande für die Jahre 1540-1800 und den Short Title Catalogue Vlandeeren (STCV), ${ }^{69}$ der die Literatur Flanderns des 17. Jahrhunderts verzeichnet. Inhaltlich erschlossen wird in diesen beiden verwandten Projekten mit Hilfe von circa 60 Deskriptoren, die auf einer Liste der Königlichen Bibliothek der Niederlande basieren. Bei der Verzeichnung bekommt jedes Werk mindestens einen dieser Deskripto$\operatorname{ren}^{70}$ (z.B. Geschichte: Deutschland), meist in Kombination mit einem Formschlagwort (z.B. Emblembuch). Mehrere Deskriptoren sind zur Vergabe möglich. So erhalten z.B. Biographien ein Formschlagwort „Biographie“, ein Geographikum gemäß des behandelten Landes und ein Zeitschlagwort für die behandelte Periode. Zusätzlich wird jedes Werk mit einem typographischen Deskriptor versehen. Die dafür herangezogene Liste enthält circa 30 Bezeichnungen. Diese geben Auskunft über Illustrationen, Schriftart und weitere Ausstattungen und Besonderheiten. ${ }^{71}$

Aufgrund der geographischen und historischen Voraussetzungen wesentlich breiter angelegt ist der English Short Title Catalogue (ESTC). ${ }^{72}$ So verzeichnet der ESTC, die retrospektive Nationalbibliographie des englischsprachigen Raums, circa 470.000 Einheiten, die in den Jahren zwischen 1473 und 1800 sowohl in Großbritannien und Nordamerika als auch in den früheren englischen Kolonien gedruckt wurden, unabhängig der Sprache. Dazu werden alle Titel in englischer Sprache verzeichnet, unabhängig inres Druckortes. Die Datenbank weist somit u.a. portugiesische Drucke aus Indien, deutsche Drucke aus Nordamerika und gälische Drucke aus Schottland nach. Belegt sind die Bestände der British Library und über 2000 weiterer Bibliotheken. Hervorge-

68 Short Title Catalogue Netherlands (2008). Online verfügbar unter http://www.kb.nl/stcn/ stcn-en.html, zuletzt geprüft am 16.02.2008.

69 Auch die rein englischsprachige Bezeichnung ist gebräuchlich: Short Title Catalogue Flanders. Siehe unter Short Title Catalogus Vlaanderen (2005). Online verfügbar unter http://www.stcv.be/, zuletzt aktualisiert am 14.02.2005, zuletzt geprüft am 16.02.2008.

70 Bei den folgenden Beispielen handelt es sich natürlich um deutsche Übersetzungen der Originalbegriffe.

71 Diese Angaben stammen aus je einer E-Mail von Goran Proot, Mitarbeiter der Universitätsbibliothek Antwerpen und Projektleiter des STCV vom 14.05.2007 und von Jan Bos, Projektleiter des STCN vom 22.05.2007.

Um Näheres zu Zielen, Inhalt und Aufbau des STCV zu erfahren, siehe Proot, Goran (2005): Ergebnisse des Short Title Catalogus Vlaanderen: Die erste Phase (2000-2003): Kirchliches Buch- und Bibliothekswesen. Jahrbuch 2004. Rottenburg am Neckar: Diözesanbibliothek der Diözese Rottenburg-Stuttgart, S. 227-249 und vor allem, inklusive einer Liste der Deskriptoren: Impe, Steven van; Rossem, Stijn van; Proot, Goran (2005): Handleiding voor de Short Title Catalogus Vlaanderen. Tweede, herziene uitgave. Antwerpen: Stadsbibliotheek Antwerpen (Armarium, 1).

72 English Short Title Catalogue (2008) Online verfügbar unter http://estc.bl.uk/F/?func=file\&file_name=login-bl-list, zuletzt geprüft am 16.02.2008 
gangen ist der ESTC 1987 aus dem „Eighteenth Century Short Title Catalogue" (ECSTC). Die inhaltliche Erschließung der Werke erfolgt mit Library of Congress Subject Headings (LCSH), ${ }^{73}$ den Schlagwörtern der Library of Congress in den USA, und mit den Genre Terms der Rare Books and Manuscripts Section (RBMS) ${ }^{74}$ der American Libraries Association ( $A L A$ ), also speziellen Gattungsbegriffen für alte Drucke, die im englischen Sprachraum angewandt werden, ähnlich denen im VD 17. Als man mit dem ECSTC begann, gab es, bis auf einige Formschlagwörter (wie z.B. prospectus, advertisement, almanac, single sheet verse) keine Verschlagwortung. Als entschieden wurde, zu diesem Katalog auch Literatur vor 1701 hinzuzunehmen und somit den ESTC zu entwickeln, wurde vereinbart, diese Literatur fortan per LCSH zu erschließen. Das Ergebnis dieser Vorgehensweise ist nun, dass zwar sämtliche Literatur vor 1701 mit Subject Headings erschlossen ist, die große Mehrheit der Literatur nach 1701 aber nicht. Dabei werden nun sämtliche Neueingänge mit LCSH ausgestattet, egal aus welchem Jahr sie stammen. Zudem wird im Moment eine rationelle Möglichkeit gesucht, mit der man die Werke des 18. Jahrhunderts retrospektiv mit LCSH verschlagworten könnte. ${ }^{75}$

Jedoch findet im Ausland auch ohne die Anlage eines speziellen Nachweissystems häufig eine fortgeschrittene Erschließung der alten Bestände statt. Als Beispiel hierzu dient die Library of Congress (LOC). ${ }^{76}$ Die LOC befindet sich in Washingon, D.C. in den USA und zählt zu den größten und bedeutendsten Bibliotheken der Welt. In ihrem Bestand befinden sich über 130 Millionen Einheiten, davon circa 30 Millionen Druckerzeugnisse und damit verbunden natürlich auch ein quantitativ riesiger Altbestand. Dieser wird genau so behandelt wie aktuelle Literatur, d.h. es wird bei der inhaltlichen Erschließung kein Unterschied gemacht. Sämtliche Literatur wird mit LCSH versehen. Nach Angabe der Bibliothek besteht der einzige Unterschied darin, dass bei historischer Literatur gegebenenfalls noch mehr Schlagwörter vergeben werden, um eine möglichst genaue Recherche zu ermöglichen. Bei gewöhnlicher und aktueller Literatur wird versucht, mit drei Schlagwörtern pro Werk auszukommen. ${ }^{77}$

Obwohl die LCSH nicht ganz so eng gefasst sind wie die RSWK, widerspricht diese Anwendung innerhalb der LOC den in Kap. 3.1. zitierten allgemeinen Ansichten Syrés, dass in Bezug auf alte Drucke eine Systematisierung, d.h. Klassifizierung, in jedem Fall

73 Eine Einführung in die Thematik und in die Anwendung der LCSH kann man einsehen unter Library of Congress Subject Headings Principles and Policies (2001). Online verfügbar unter http://www.itsmarc.com/crs/shed0014.htm, zuletzt aktualisiert am 28.02.2001, zuletzt geprüft am 16.02.2008.

74 Ebenfalls eine Einführung sowie die Liste der Genre Terms kann eingesehen werden unter: RBMS Controlled Vocabularies. Genre Terms (2007). Online verfügbar unter http://www.rbms.info/committees/bibliographic_standards/controlled_vocabularies/genre/alphabetical_list.htm, zuletzt aktualisiert am 30.08.2007, zuletzt geprüft am 16.02.2008.

75 Diese Angaben stammen aus einer E-Mail von Simon May, Mitarbeiter der British Library vom 15.05.2007

76 The Library of Congress (2008). Online verfügbar unter http://www.loc.gov/index.html, zuletzt geprüft am 16.02.2008.

77 Diese Angaben stammen aus einer E-Mail von David Williamson, Mitarbeiter der Library of Congress vom 15.05.2007 
einer Schlagwortvergabe vorzuziehen wäre, da es keine brauchbare Methode der verbalen Sacherschließung für alte Drucke gäbe. Wie das Beispiel Library of Congress zeigt, ist es wohl doch möglich, Altbestände auch mit Hilfe eines modernen Schlagwortsystems zu erschließen. 


\section{Die Erschließungssituation in ausgewählten Bibliotheken}

Die Daten der vorliegenden Betrachtungen wurden aus verschiedenen Quellen zusammengetragen. Zum einen wurde Sekundärliteratur ausgewertet und die Internetpräsenz der jeweiligen Bibliothek betrachtet, zum anderen wurde den Bibliotheken zusätzlich ein Fragebogen mit der Bitte um Beantwortung zugesandt. Die Fragen bezogen sich auf die jeweilige Erschließungspraxis innerhalb der Bibliothek. ${ }^{78}$ Aus diesen Antworten stammt auch der Großteil der hier zitierten Angaben. Im folgenden Abschnitt wurde deshalb auf eine jeweilige Quellenangabe verzichtet, es sei denn, es wurde zusätzlich Fachliteratur zitiert.

\subsection{Universitätsbibliothek Eichstätt}

Die jüngst durch die sogenannte "Causa Eichstätt“ in das Interesse der öffentlichen Wahrnehmung gerückte Bibliothek erschließt antiquarische Neuerwerbungen ebenso wie auch sonstige Erwerbungen per Regensburger Verbundklassifikation. Eine Verschlagwortung findet nicht statt. Der bereits vorhandene Altbestand ist nach einer hauseigenen Gruppensystematik aufgestellt. Die Standortsignaturen dienen zugleich zur Sacherschließung, wobei diese nur eher grob erschließen.

Die UB Eichstätt befasst sich zudem seit mehreren Jahren mit der Einarbeitung sehr umfangreicher Altbestände aus dem Vorbesitz der bayerischen Kapuziner und der Münchener Jesuiten. Diese Altbestände (bis Erscheinungsjahr 1800) erhalten neben einem Numerus Currens für die Aufstellung auch eine Notation zur Sacherschließung, die auf der genannten hauseigenen Gruppensystematik basiert.

Der Aufwand dieser Erschließung wird von den Verantwortlichen als vertretbar und der Nutzen als unbedingt lohnend eingeschätzt. Man will diese Form der Erschließung weiterführen, ausbauen will man sie jedoch nicht.

78 Fragebogen S. S. 98 


\subsection{Staats- und Universitätsbibliothek Hamburg}

Als Mitglied der Arbeitsgemeinschaft Alter Drucke im GBV (AAD) erschließt die SUB Hamburg ihre Altbestände seit Januar 2006 sachlich mit Gattungsbegriffen. Als Grundlage hierfür dient die Schlagwortliste der AAD. Diese kombiniert die sogenannte "Göttinger"-Liste mit der VD17-Liste. ${ }^{79}$ Nach Ansicht der Bibliothek überwiegen die Vorteile, die man mit der Vergabe von Gattungsbegriffen erlangt, die Nachteile in Form des Aufwandes. Dabei kann durchaus auch auf die Vergabe verzichtet werden, sollte der Aufwand einmal als nicht mehr vertretbar erscheinen.

Eine komplette Sacherschließung, die bei alten Drucken grundsätzlich nach den gleichen Regeln wie für moderne Bestände erfolgt (z.B. RSWK, Klassifikation), ist für die Bibliothek zu aufwändig und wird nur vereinzelt vorgenommen. Als Ursachen hierfür werden die bekannten Gründe genannt, u.a. dass diese Titel häufig in lateinischer Sprache seien, sie schwierige Druckbilder besäßen und zudem teilweise Wissenschaftszweige behandelten, die so heute nicht mehr existieren. Die Ermittlung des jeweiligen Inhalts gerät dadurch schwer und zeitaufwändig. Als ihre primäre Aufgabe sieht es die Bibliothek an, die Bestände überhaupt elektronisch nachzuweisen. Bei einem großen Teil der Werke geschieht die Katalogisierung anhand retrospektiver Konversion. Nur bei Drucken des 16./17. Jahrhunderts sowie bei besonders wertvollen und seltenen Werken wird per Autopsie katalogisiert. Es wird indes nicht ausgeschlossen, dass zukünftig in bestimmten Bestandsbereichen durch Digitalisierungsprojekte eine tiefere inhaltliche Erschließung vorgenommen wird.

Neben einem konvertierten Alphabetischen Zettelkatalog, in dem circa 800.000 Veröffentlichungen mit Erscheinungsjahr vor 1974 enthalten sind, ${ }^{80}$ existieren auch ein Alter Schlagwortkatalog ${ }^{81}$ und ein Alter Systematischer Katalog ${ }^{82}$ der Sondersammelgebiete für Bestände bis 1995.

79 Die Liste lässt sich einsehen unter: AAD Gattungs- und Sachbegriffe (2005). Online verfügbar unter http://aad.gbv.de/empfehlung/aad_gattung.pdf, zuletzt aktualisiert am 14.12.2005, zuletzt geprüft am 17.02.2008.

80 Wovon circa 40\% Prozent bereits im elektronischen Campus-Katalog nachgewiesen sind. Stand: 17.02.2008

81 Ein Schlagwortkatalog (Alphabetischer Sachkatalog) „ist ein Sachkatalog, in dem die Büchertitel unter Begriffswörtern (Schlagwörtern) eingeordnet werden, die aus dem jeweils vorliegenden Buch gewonnen werden. Diese Begriffswörter werden unter sich alphabetisch geordnet." Vgl. Leyh, Georg (1961): Handbuch der Bibliothekswissenschaft. Begründet von Fritz Milkau. Band 2: Bibliotheksverwaltung. 2., verm. und verb. Auflage. Wiesbaden: Harrassowitz, hier S. 96

82 Ein Systematischer Katalog „verzeichnet [...] den Bestand einer Bibliothek nach dem Inhalt der Publikationen." Und zwar im Gegensatz zum Schlagwortkatalog nicht nach Schlagwörtern, sondern nach Wissensgebieten. „Die Ordnung der einzelnen Wissensgebiete erfolgt in einer sachlich-logischen Reihenfolge, innerhalb der Wissensgebiete nach dem jeweiligen Klassifikationssystem, das meist eine hierarchische Ordnung von Haupt- und Unterklassen kennt. Die Klassen werden durch eine Notation (meist alphanumerisch) bezeichnet." Corsten, Severin; Bischoff, Bernhard (1987-2004): Lexikon des gesamten Buchwesens. LGB 2. 2., völlig neubearb. Aufl. Stuttgart: Hiersemann. Lieferung 53: Synagoge - Thekenbücherei (Band 7. Seiten 321-400), hier S.329. 


\subsection{Staats- und Universitätsbibliothek Göttingen}

Der Altbestand der Staats- und Universitätsbibliothek Göttingen (SUBG), der circa 800.000 Bücher und Zeitschriften bis 1945 umfasst und weitestgehend im OPAC der SUBG erfasst ist, ist komplett erschlossen durch den Göttinger Realkatalog. ${ }^{83}$ Dessen Systematik untergliedert sich in elf Hauptgruppen, die wiederum in 30.000-40.000 Klassen gegliedert sind. Die kleinste Systemstelle entspricht dabei der Signatur des jeweiligen Titels. Eine thematische Online-Recherche, die ebenso funktioniert wie eine Recherche direkt im Realkatalog, ist dabei über die „Systematische Suche bis 1945“84 möglich, wobei nicht einzelne Titel sondern einzelne Systemstellen durch Schlagwörter erschlossen sind.

Unterteilt ist der Realkatalog in folgende elf Hauptgruppen:

I. Encyclopaedia

II. Historia Literaria

III. Theologia

IV. Jurispudentia \& Politica

V. Historia \& Geographia

VI. Philosophia

VII. Artes

VIII. Literatura et Linguistica

IX. Mathematica et Scientiae naturalis

X. Medicina

XI. Sonstige angewandte Naturwissenschaften

Allgemeine Neuerwerbungen werden ebenso wie Neuerwerbungen für die Sammlung Deutscher Drucke, für deren Zeitsegment 1701-1800 die SUB Göttingen bekanntlich zuständig ist (Vgl. Kap. 3.1.), laufend per Basisklassifikation und Gattungsbegriff erschlossen. Diese Erschließungsform dauert nach Angaben der Bibliothek pro Druck zwischen 5 Sekunden und 5 Minuten. Am VD 17 ist die Bibliothek ebenfalls beteiligt. Die dafür relevanten Drucke werden im Rahmen der VD 17-Regularien erschlossen, d.h. bei der sachlichen Erschließung wird nur mit Gattungsbegriffen gearbeitet.

Ihr Vorgehen bezeichnet die Bibliothek durchaus als lohnenswert, da sachliche Recherchen im Bereich der Altbestände von großer Bedeutung seien. Die Verantwortlichen der SUBG gehen davon aus, dass bei bibliographischen Großprojekten inhaltlich auch zukünftig hauptsächlich mit Gattungsbegriffen erschlossen werden wird.

83 Realkatalog ist eine, vor allem im 19. Jahrhundert, bevorzugte Bezeichnung für einen Systematischen Katalog.

84 Niedersächsische Staats- und Universitätsbibliothek (2008). bandrksyst. Online verfügbar unter http://opac.sub.uni-goettingen.de/DB=1/BANDRKSYST, zuletzt geprüft am 17.02.2008. 


\subsection{Staatsbibliothek zu Berlin}

In der Staatsbibliothek zu Berlin befindet sich mit circa 3 Millionen Bänden der größte historische Druckschriftenbestand Deutschlands. Durchgehend inhaltlich-sachlich erschlossen sind diese zwischen 1501 und 1955 erschienenen Titel im Alten Realkatalog (ARK). Jeder Titel besitzt eine Signatur und ist über diese auch gezielt auffindbar. Diese Signaturen spiegeln die systematische Einordnung des Titels im ARK wider. Angelegt wurde der Bandkatalog im 19. Jahrhundert, erarbeitet wurde er in den Jahren 1842 bis 1881. Die Klassifikation des ARK bildet die geschichtlichen Umstände und den Stand der Wissenschaftsentwicklung Mitte des 19. Jahrhunderts ab. So entspricht die Abfolge der einzelnen Wissenschaftsdisziplinen innerhalb des Katalogs der Reihenfolge der Universitätsfakultäten. Auf die Theologie folgt die Rechtswissenschaft, darauf die Medizin und zuletzt die Philosophie. An den Beginn wurde zusätzlich eine allgemeine Abteilung gestellt. Weitere Fächer, die Anfang des 19. Jahrhunderts noch nicht an Universitäten gelehrt wurden, wurden nachträglich eingeschoben. Insgesamt besteht der ARK nun aus sechzehn Teilgebieten.

Meist wurden die originären historischen Bezeichnungen übernommen und bei Bedarf um zusätzliche Begriffe ergänzt. Zudem wurden diese bei Verwendung verschiedener Bezeichnungen für den selben Sachverhalt normiert, um Recherchen zu erleichtern. Zusätzlich wurden für alle Systemstellen Schlagworte vergeben. Diese beschreiben entweder den Gesamtinhalt oder Teilinhalte der jeweiligen Systemstelle. Die Schlagwörter basieren in der Regel auf Grundlage der SWD. Zusätzlich zu den Schlagwörtern wurden für alle Systemstellen auch Notationen der Basisklassifikation angelegt. Diese sind ebenfalls recherchierbar.

Im Moment wird die Systematik des ARK in Form eines Projektes vollständig aufbereitet, elektronisch erfasst und online zur Verfügung gestellt. Man kann sowohl in der Systematik blättern als auch mit Suchbegriffen innerhalb der Systematik recherchieren. Im Moment sind 40\% der Online-Systematik durchsuchbar. ${ }^{85}$

Zusätzlich zu seiner eigentlichen Katalogfunktion ist der ARK ein fachbibliographisches Nachweisinstrument von weltweiter Bedeutung. ${ }^{86}$

85 Stand: 27.02 .2008

86 Vgl. ARK Sachkatalog der Staatsbibliothek zu Berlin - Preußischer Kulturbesitz (2008). Online verfügbar unter http://ark.staatsbibliothek-berlin.de/index.php, zuletzt geprüft am 17.02.2008. 


\subsection{Herzogin Anna Amalia Bibliothek Weimar}

In der durch den verheerenden Brand $^{87}$ vom 2. September 2004 stark in Mitleidenschaft gezogenen Herzogin Anna Amalia Bibliothek (HAAB) in Weimar sind die Altbestände bis auf wenige Ausnahmen in Zettelkatalogen verzeichnet. Erschlossen sind sie mit speziellen, hauseigenen Regelwerken. Vorhanden sind ein allgemeiner Schlagwortkatalog und eine Reihe von systematischen Sonderkatalogen für spezielle Bestandsgruppen.

Der vorhandene Altbestand der HAAB ist inhaltlich bereits fast vollständig erschlossen, es werden lediglich nur noch antiquarische Neuerwerbungen sachlich bearbeitet. Da nach besagtem Bibliotheksbrand durch Ersatzbeschaffung und Geschenk sehr viel Altbestand innerhalb kurzer Zeit in die Bibliothek kommt ${ }^{88}$ und dadurch die Kapazität der Sacherschließung im Moment überfordert ist, werden diese Bestandsgruppen zur Zeit nicht bearbeitet. Jedoch werden diese mit einem entsprechenden Code in der Titelaufnahme versehen, so dass sie später problemlos nachbearbeitet werden können, wenn die Umstände wieder positiver erscheinen.

Der allgemeine, d.h. nicht spezielle Bestand bis Erscheinungsjahr 1995 wird im allgemeinen Schlagwortkatalog sachlich erschlossen. Verzeichnet wird alphabetisch nach Schlagworten, innerhalb eines Schlagwortes chronologisch absteigend nach den Erscheinungsjahren. Angewandt wird ein hauseigenes Regelwerk, dass sich in vielen Punkten von den RSWK unterscheidet. So gibt es u.a. Haupt-, Unter- und Nebenschlagwörter. Teilweise existieren weite und enge Schlagwörter nebeneinander, wobei die Unter- und Nebenschlagwörter nur teilweise normiert sind.

Der für die HAAB sehr wichtige spezielle Bestand (z.B. deutsche Literatur, v.a. der deutschen Klassik, Faust-Sammlung, Liszt-Bibliothek, Nietzsche-Bibliothek) wird extra in systematischen Sonderkatalogen erschlossen ${ }^{89}$ und überschneidet sich nicht mit dem Bestandsnachweis im Schlagwortkatalog.

Mit einem standortfreien Realkatalog aus der Mitte des 18. Jahrhunderts fand die älteste inhaltliche Erschließung der Bestände statt. Dieser wurde von 1754 bis 1830 geführt, teilweise auch bis Anfang des 20. Jahrhunderts. Es finden sich gelegentlich noch Einträge bis zum Erscheinungsjahr 1945.

87 Circa 50.000 Bücher wurden dabei unwiederbringlich zerstört, weitere 62.000 Bände konnten nur noch beschädigt geborgen werden. Im Mai 2006 zählt der Bibliotheksbestand ungefähr 900.000 Bände. Nähere Informationen hierzu gibt es unter: Hilfe für Anna Amalia (2007). Online verfügbar unter http://www.anna-amalia-bibliothek.de/de/afterbrand.html, zuletzt aktualisiert am 14.12.2007, zuletzt geprüft am 17.02.2008.

88 Man geht davon aus, dass drei Viertel der zerstörten 50.000 Bände langfristig wieder zu beschaffen sind. Im Oktober 2007 waren circa 4000 Bände des historischen Bestandes bereits wieder erworben worden. Man muss jedoch damit rechnen, dass sich der Erwerbungsprozess über mehrere Jahrzehnte hinziehen und stark von den vorhandenen Geldmitteln abhängen wird. Vgl. Hilfe für Anna Amalia (2007)

89 Bis Erscheinungsjahr 2002 
Er umfasst 60 Bände und ein dazugehöriges Stich- und Schlagwortregister. Unterteilt ist der Bandkatalog in sieben Hauptgruppen:

- Bibliotheca theologica

- Bibliotheca iuridica

- Bibliotheca physico-medica

- Bibliotheca historica

- Bibliotheca philosophica

- Bibliotheca philologico-critica

- Bibliotheca technica et opificaria
6 Bde, 1 Register

12 Bde, 1 Register

5 Bde, 1 Register

2 Bde, 1 Register

2 Bde, 1 Register

4 Bde, 1 Register

2 Bde, 1 Register

Der Realkatalog ist mikroverficht und die Mikrofiches sind dem Benutzer zugänglich. Daneben entstanden eine Reihe von weiteren Katalogen, Sonder- und Teilkatalogen. ${ }^{90}$

Die Anfänge des Schlagwortkatalogs in der heutigen Form gehen zurück nach 1949. Ab 1969 gab es ein großes Katalogisierungs- und Konversionsprojekt, bei dem mehrere Kataloge und Bestände zusammengeführt wurden. Dieses Projekt zog sich über fast 25 Jahre hin. In diesem Zusammenhang erfolgte auch die neue sachliche Bearbeitung des gesamten Bestandes, sowohl systematisch in den Sonderkatalogen als auch verbal für den Schagwortkatalog. Für die Bibliothek war dieses Projekt nach eigener Aussage ein Glücksfall, da dadurch fast der gesamte Altbestand nach einheitlichen Kriterien inhaltlich erschlossen wurde.

Bei der Konversion des alten alphabetischen Zettelkatalogs (PI-Katalog) in PICA in den 90er Jahren ${ }^{91}$ wurden auch die Sachvermerke manuell mit eingegeben und sind somit im OPAC der Bibliothek vorhanden. Da dabei nur die reinen Sachvermerke übertragen wurden, existiert im OPAC weder eine Strukturierung wie im Zettelkatalog noch gibt es Normdaten. Gerne würde die Bibliothek diese Sachvermerke der Zettelkataloge in strukturierter und nutzerfreundlicher Aufbereitung anbieten, jedoch wird dies zur Zeit als nicht realisierbar eingeschätzt.

Ebenfalls als nicht möglich angesehen wird aktuell eine weitere Erschließung als über den eben dargestellten Rahmen hinaus. Die Bibliothek hofft, irgendwann einmal die Titel sachlich erschließen zu können, die im Moment den Code „nicht sacherschlossen“ erhalten haben. Neuerworbene Titel mit Erscheinungjahr vor 1996 (Schlagwortkatalog) bzw. 2002 (Systematische Kataloge) erhalten zur Zeit noch einen Ausdruck, der in die Zettelkaloge eingestellt wird. So verzeichnen diese Kataloge den Altbestand bis zum jeweiligen Erscheinungsjahr fast vollständig (mit den bereits erwähnten Ausnahmen).

90 Weitere Bandkataloge sind z.B. der Catalogus nominalis (dieser besteht aus 37 Bänden, geordnet alphabetisch nach Verfassern, geführt wurde er von 1776 bis 1928) und der Catalogus anonymorum (dieser hingegen besteht aus 19 Bänden, die systematisch nach Titelstichwörtern oder Schlagwörtern geordnet sind, gelegentlich gibt es eine weitere Unterteilung bei Städten und Ländern, geführt wurde er von 1776 bis 1928.)

91 Eingearbeitet wurde bis Erscheinungsjahr 1850 sowie ab 1977 vollständig. 
Es ist inzwischen jedoch zweifelhaft, ob dies in Zukunft weiterhin so gehandhabt werden kann. Dieser Zuwachs in den Zettelkatalogen betrifft aber eher selten ältere Bestände sondern vorwiegend Literatur des zwanzigsten Jahrhundert.

Eine heutige, vollständige sachliche Retrokatalogisierung der Altbestände wird von der HAAB vor allem für die großen Bibliotheken als unmöglich angesehen. Auf Grund der geringen, bzw. kaum vorhandenen Normierung der inhaltlichen Erschließungsdaten in der Vergangenheit sei auch eine Übernahme von Fremddaten anderer Bibliotheken nur mit vielen Abstrichen und sehr großem manuellem Aufwand möglich. Man neigt dazu, diesen Aufwand als nicht umsetzbar einzuschätzen.

Überlegungen in den 1990er Jahren, die Sachkataloge mit Hilfe von Images zu konvertieren, wie dies z.B. in der Zentral- und Landesbibliothek Berlin und in der Universitätsund Landesbibliothek Sachsen-Anhalt in Halle gehandhabt wurde, wurden aufgrund mangelnder qualitativer Überzeugung wieder verworfen. ${ }^{92}$

\subsection{Herzog August Bibliothek Wolfenbüttel}

Die Herzog August Bibliothek Wolfenbüttel (HABW), deren langjähriger Leiter der bereits zuvor zitierte Paul Raabe war, hat einen Teil ihres Altbestandes der Jahre zwischen 1472 und 1830 systematisch erschlossen. ${ }^{93}$ Erschlossen wurden dabei die Fachgebiete Medizin, Naturwissenschaften, Jurisprudenz und Mathematik aus den Jahren 1472-1830 vollständig (ca. 45.000 Titel in den Jahren 1977-1985), die Theologie zu einem großen Teil (ca. 80.000 Titel in den Jahren 1986-1991). Doch konnte dies aus finanziellen Gründen nicht zu Ende geführt werden. Um den Inhalt der Bücher auch wirklich bestimmen zu können, wurde die Erschließung per genereller Autopsie durchgeführt. Zu diesem Zweck wurden die Bücher entweder aus den Magazinen geholt oder die Mitarbeiter arbeiteten direkt in den Magazinen. Bei der Realisierung handelte es sich um zwei aufeinanderfolgende Projekte, die zwischen 1977 und 1991 durchgeführt und aus Drittmitteln finanziert wurden.

Benutzt wurde aufgrund des Alters der Bestände das „Hallesche Schema“, eine Systematik des 19. Jahrhunderts, welche 1888 von Otto Hartwieg für die UB Halle enwickelt wurde. Ergänzt wurde sie durch die Integration von Schlagwörtern. Diese wurden an den Lexika ihrer Zeit kontrolliert und sind daher annehmbar normiert, jedoch lässt sich, nach Aussage der Bibliothek, z.B. nicht für jeden Krankheitsbegriff des 16. Jahrhunderts eine gesicherte Definition finden.

Der Zeitaufwand einer solchen Erschließungsform wird von der Bibliothek als hoch angegeben, da die komplexen Inhalte oft durch den Einsatz von Hilfsmitteln bearbeitet

92 Vgl. zu dem Realkatalog der HAAB auch Seifert, Siegfried (1992): „Niemand wird läugnen, daß ein Real-Catalog das Fundament einer jeden Bibliotheks-Anstalt sey". Bemerkungen zum historischen Realkatalog der Weimarer Republik. In: Kratzsch, Konrad; Seifert, Siegfried (Hg.): Historische Bestände der Herzogin-Anna-Amalia-Bibliothek zu Weimar. Beiträge zu ihrer Geschichte und Erschließung ; mit Bibliographie. München: Saur (Literatur und Archiv, 6).

93 Vgl. dazu auch Arnold, Werner (1997) 
werden müssen. Zudem treten zahlreiche verschieden Sprachen auf, die die Bearbeitung der einzelnen Werke zusätzlich erschwert und zeitlich beansprucht, so z.B. Deutsch, Latein, Griechisch, Französisch, Italienisch, Englisch und weitere Sprachen, diese jedoch in geringerem Umfang. Aus den Gebieten Medizin, Naturwissenschaften, Jurisprudenz und Mathematik wurden im Jahr ca. 6.000 Titel erschlossen, bei der Theologie waren es ca. 10.000, wobei sich der verantwortliche Mitarbeiter dort ganz auf seine Aufgabe konzentrieren konnte. Streng am ökonomischen Maßstab gemessen ist der Aufwand einer solchen Vorgehensweise nach Auskunft der Bibliothek vermutlich zu hoch, um ein Musterbeispiel zu sein. Dabei ist das Ergebnis inhaltlich sehr positiv und zufriedenstellend für die Wissenschaft. Diese nutzt die inhaltlich erschlossenen Bestände intensiv.

Die konventionellen Erschließungsdaten für Medizin und Naturwissenschaften wurden 2006/2007 in den OPAC integriert, so dass darin eine Recherche mit Hilfe der Systematik möglich ist. Die vorliegenden juristischen Daten sollen ebenfalls integriert werden. Zudem wird in der Bibliothek im Moment darüber diskutiert, in welcher Art man die weitere inhaltliche Erschließung der Altbestände fortsetzen soll. Es besteht noch keine Einigkeit darüber, ob die Erschließung nur verbal oder systematisch mit integrierten verbalen Elementen durchgeführt werden soll.

Obwohl diese Erschließung sehr zeitaufwendig und kostenintensiv ist, gibt es nach Ansicht der Bibliothek keinen überzeugenden Grund, warum man auf sie verzichten sollte.

\subsection{Bayerische Staatsbibliothek}

Die Bayerische Staatsbibliothek erschließt ihren Altbestand in zwei verschiedenen Katalogen. Zum einen verbal im ersten Schlagwortkatalog der Bibliotheksgeschichte, dem sogenannten Schrettinger-Katalog. Dieser wurde gepflegt in den Jahren 1819-1856, darin erschlossen sind die Erscheinungsjahre 1501-1856. Zum anderen systematischim im Alten Realkatalog. Dieser besteht aus drei Gruppen, Geographischer- oder Länderteil, Sachlicher- oder Historischer Teil und Kunstteil. Der Länderteil bildet mit rund $80 \%$ den Hauptbereich und spiegelt die geographischen Verhältnisse um 1870 wider. Die Laufzeit des Realkatalogs begann um 1870 und geht durch die Einarbeitung laufender Antiquaria bis in die Gegenwart. Verzeichnet werden in inm die Erscheinungsjahre 1501-1856. Zudem existieren zahlreiche Spezialkataloge für Musik, Orient, Osteuopa und Karten sowie für Handschriften und Inkunabeln. Dabei wurde die Literatur ab 1501, die die Bibliothek vor Inbetriebnahme der Kataloge erworben hatte, in großen Teilen rückwirkend eingearbeitet.

Die Erschließung erfolgt im Schrettinger-Katalog mit Hilfe von Schlagwörtern und gegenseitigen Siehe-auch-Verweisungen. Dabei wird das „Prinzip des engsten Schlagwortes" angewandt. Die verwendeten Schlagwortkategorien sind Personenschlagwörter, Geographische Schlagwörter und Sachschlagwörter. Eine weitere Kategorie bilden Institutionen. Der Alte Realkatalog beruht auf einer hausinternen Klassifikation, die sich in der Tiefengliederung am Bestand der Bibliothek orientiert und auf der Wissenschaftsstruktur von circa 1870 basiert. 
Der Aufwand lässt sich dabei nicht genau festlegen, jedoch ist die Bibliothek davon überzeugt, dass der qualitative Nutzen der Erschließung in jedem Fall den quantitativen Aufwand übersteigt. Erwähnenswert ist, dass die BSB mit ihren beiden historischen Katalogen neben der Staatsbibliothek Berlin die einzige Bibliothek im gesamten deutschen Sprachgebiet mit durchgehender und umfassender Sacherschließung ihres Altbestands über einen Zeitraum von mehr als 450 Jahren ist. Zusammen mit der laufenden Sacherschließung der aktuellen Literatur gilt dies bis in die Gegenwart. Es wird davon ausgegangen, dass der Mehrwert der Kataloge im Rahmen der Fachportale weiter gesteigert wird. Eine Konversion der beiden Kataloge wurde ebenfalls beantragt, aber aus finanziellen Gründen bisher noch nicht bewilligt. Als große Magazinbibliothek mit einem Bestand von über acht Millionen Bänden will die Bibliothek keinesfalls auf Sacherschließung der Altbestände verzichten. Diese wird eher noch weiter ausgebaut, z.B. in Form von einzelnen Erschließungs- oder Konversionsprojekten. ${ }^{94}$

\section{8 Österreichische Nationalbibliothek}

Die Österreichische Nationalbibliothek (ÖNB) ist die größte Bibliothek Österreichs und in dieser Untersuchung die einzige außerhalb der Grenzen Deutschlands.

Unbedingt erwähnenswert an der ÖNB ist, dass dort sämtliche Altbestände mit Hilfe von Schlagwörtern inhaltlich erschlossen wurden und nach wie vor werden. Dieser Umstand schlägt sich auch deutlich in den in Kapitel 5.3. durchgeführten Untersuchungen nieder. Einzig bestimmte Teile von Spezialsammlungen sind von dieser Erschließungspraxis ausgenommen.

Von 1967 bis 1997 wurden mittels einer eigens dafür geschaffenen Abteilung die alten Drucke retrospektiv beschlagwortet, in den meisten Fällen jedoch ohne Autopsie. Diese Erschließung wurde nach den Hausregeln der ÖNB von 1970 (1984 ergänzt) vorgenommen, basierend auf den schon 1923 von der ÖNB herausgegebenen Regeln für die Schlagwortvergabe. Die Belletristik wurde dabei zusätzlich zur inhaltlichen ErschlieBung in einem Spezialkatalog nach literarischer Gattung, Sprache und Jahrhundert erschlossen. Alle formalen und inhaltlichen Erschließungsdaten wurden Ende der 90er Jahre in einem maschinenlesbaren Katalog zusammengefasst. Die Inhalte dieses Kataloges werden laufend adaptiert, korrigiert und ergänzt. Die notwendigen Nachbearbeitungen und Korrekturen unzutreffender Schlagwörter bzw. die Vergabe von fehlenden Schlagwörtern bei bisher noch nicht oder unzureichend erschlossenen Titeln werden bei konkretem Anlass ebenfalls bis zum heutigen Tag meist autoptisch durchgeführt.

Neuanschaffungen alter Drucke werden bereits seit den späten 60ern Jahren durchgehend anhand Autopsie inhaltlich erschlossen. Seit 1990 findet die Katalogisierung in ei-

94 Vgl. zu den Katalogen der Bayerischen Staatsbibliothek auch Rückert, Ingrid; Groß, Matthias (2003): Der Alte Realkatalog der Bayerischen Staatsbibliothek und sein neues Online-Register. In: Bibliotheksforum Bayern, Jg. 31, H. 3, S. 214-223 sowie Rückert, Ingrid (2003): Der Schrettinger-Katalog und der Alte Realkatalog. Zweihundert Jahre Sacherschließung an der Bayerischen Staatsbibliothek. München: Selbstverlag. 
nem Bibliothekssystem statt und die Beschlagwortung erfolgt seit ihrer Einführung nach der RSWK/SWD. Schöne Literatur wird durch Gattungsschlagwörter erschlossen, ebenfalls nach den RSWK. Zusätzlich werden in einer groben Systematik im Verbundsystem eine Fachgruppennummer und im lokalen System eine oder mehrere Sachgruppennummern vergeben. Diese Vorgehensweise befindet sich jedoch gerade im Umbruch. Für alte Drucke werden fakultativ lokale Stich- bzw. Schlagwörter vergeben, z.B. für Hochschulschriften; oder es wird zusätzlich eine engere Erschließung durchgeführt. Zusätzlich werden in einer Pilotphase seit 2007 Neuanschaffungen alter Drucke optional nach der Basisklassifikation erschlossen.

Der Aufwand der retrospektiven Erschließung war nach Ansicht der Bibliothek aufgrund der fehlenden Autopsie durchaus lohnend, wenn auch sehr erheblich. Man ist der Meinung, dass mit dem heutigen Stand der EDV der Aufwand um ein vielfaches geringer wäre.

Für die Zukunft ist ein Abgleich der historischen Beschlagwortung mit der SWD geplant. Eine Machbarkeitsstudie wurde bereits durchgeführt. Diese brachte für die Bibliothek ermutigende Ergebnisse. Bis zu $80 \%$ der beschlagworteten Titel sind durch entsprechende Prozeduren maschinell unterstützt zu bearbeiten. Zudem werden Neuanschaffungen alter Drucke weiterhin nach den RSWK sacherschlossen. Die weitere Verwendung der Basisklassifikation in ausgebauter Form ist ebenso geplant 


\section{Die Erschließungssituation innerhalb allgemeiner Bibliothekskataloge}

\subsection{Methodik}

Die folgende Untersuchung wurde anhand der Bestände der Öttingen-Wallersteinschen Bibiothek an der Universitätsbibliothek Augsburg durchgeführt. Speziell und exemplarisch wurde dabei die Systematikgruppe IV.18 (Geschichte der skandinavischen Reiche) untersucht. Dafür wurde die komplette Bestandsgruppe zur Begutachtung herangezogen. Insgesamt waren dies 209 Titel in 276 Bänden. Während der Auswertung wurde folgendermaßen vorgegangen:

Es wurde jeder Titel daraufhin überprüft,

- welche inhaltserschließenden Daten (Schlagwörter, Titelstichwörter, Notationen usw.) schon in den Katalogisaten der UB Augsburg vorhanden und nutzbar sind

- ob dieselben Titel in Bibliotheken und Verbünden des deutschsprachigen Raumes vorhanden und inhaltlich erschlossen sind. Dies wurde anhand des Dreiländerkatalogs und des Karlsruher Virtuellen Katalogs durchgeführt. Lokale historische Klassifikationen wurden hierbei nicht berücksichtigt.

- mit welchen Daten eine möglichst effiziente Auffindbarkeit nach inhaltlichen Aspekten erzielt werden kann.

Die gewonnenen Daten wurden, soweit möglich, graphisch aufbereitet und werden im folgenden präsentiert, wobei einzelne Titel durchaus in mehreren Kategorien enthalten sein können.

Bei der Durchführung der Untersuchung stellte sich als Problem heraus, dass die einzelnen Titel in Bezug auf Ansetzung, Orthographie usw. in den verschiedenen Bibliotheken und Verbünden teils unterschiedlich in den jeweiligen Katalogen verzeichnet sind. So ist es durchaus möglich, dass bei der Recherche nach einem Titel auch bei mehreren, orthographisch veränderten Suchanfragen nicht alle relevanten Ergebnisse herausgefiltert werden konnten. 


\subsection{Dreiländerkatalog}

Zuerst wurde mit Hilfe des Dreiländerkatalogs untersucht, wie viele Titel der Systematikgruppe IV.18 in den Katalogen dreier bedeutender Altbestandsbibliotheken enthalten sind. Anschließend folgt eine Zählung der Titel, die bereits per Schlagwort oder per RVK erschlossen sind. Über den Dreiländerkatalog sind die Daten des HBZ, des BVB, des Österreichischen Bibliothekenverbundes (OBV.SG) und des GBV recherchierbar. Insgesamt weist er über 60 Millionen Titeldaten nach. Ziel der Verantwortlichen ist es, zukünftig weitere Bibliotheksverbünde des deutschsprachigen Raumes in den DLK zu integrieren, um irgendwann alle Bibliotheksbestände aus diesem Gebiet nachzuweisen. Zu der Technologie, die hinter dem DLK steht, folgt näheres im Kapitel 7.2. ${ }^{95}$

\subsubsection{Vorkommen der Titel}

Es wurde untersucht, in welchen der drei folgenden Altbestandsbibliotheken die Titel wie häufig vorhanden sind. Dies ist von Interesse, da alle drei Bibliotheken ihren jeweiligen Altbestand durchgehend mit Hilfe einer alten Systematik klassifizieren und diese Klassifikationen eventuell für eine bibliotheksübergreifende Recherche in Form von Konkordanzen genutzt werden können (Siehe Kap. 7.3). Getestet wurden die SUB Göttingen, die StaBi Berlin und die ULB Halle.

\begin{tabular}{|c|c|c|c|}
\hline Titel insgesamt & SUB Göttingen & StaBi Berlin & ULB Halle \\
\hline 209 & 113 & 147 & 101 \\
\hline In Prozent & $54,07 \%$ & $70,33 \%$ & $48,33 \%$ \\
\hline
\end{tabular}

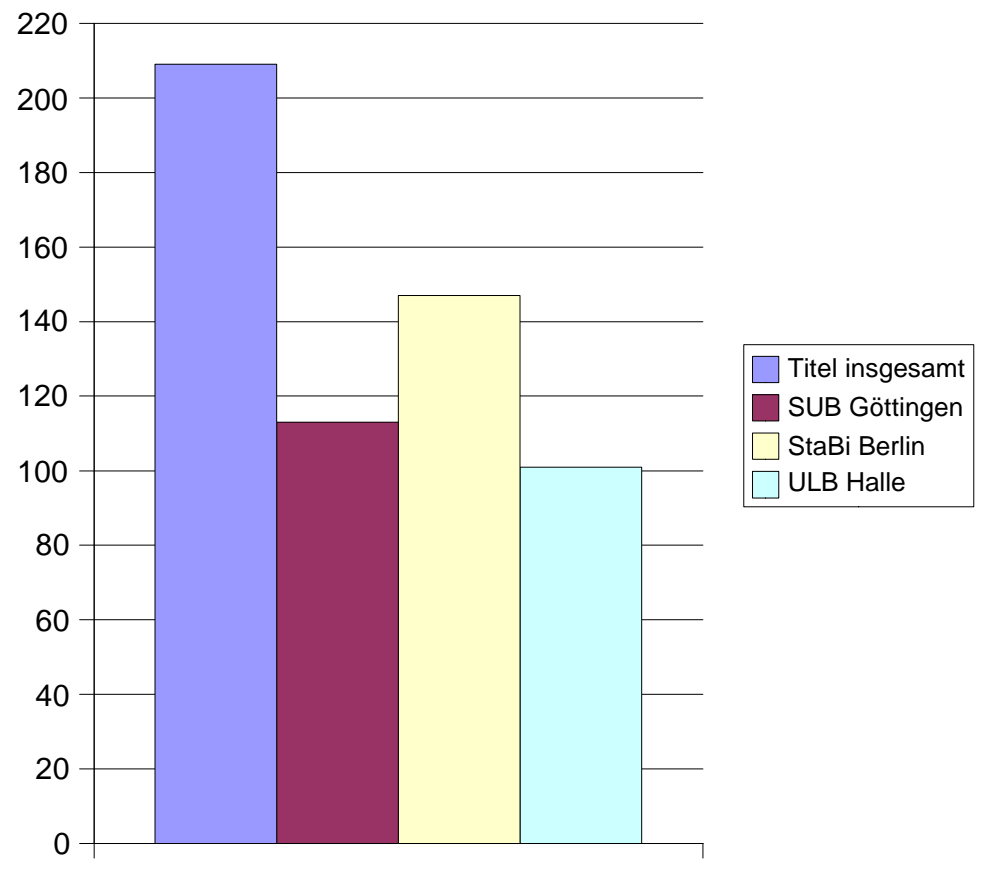

95 Dreiländerkatalog (2006). hbz. Online verfügbar unter http://www.hbz-nrw.de/angebote/dlk/, zuletzt aktualisiert am 24.11.2006, zuletzt geprüft am 29.05.2007. 
Es zeigt sich, dass die Titel relativ häufig in den drei berühmten Altbestandsbibliotheken vorhanden sind. Die wenigsten befinden sich in Halle, jedoch sind es auch dort noch beinahe $50 \%$ der ausgewerteten Werke. Zudem muss dabei bedacht werden, dass die Geschichte der skandinavischen Reiche eine eher spezielle Bestandsgruppe ist.

\subsubsection{Titel mit RVK-Notation}

\begin{tabular}{|c|c|c|}
\hline Titel insgesamt & Mit RVK erschlossen & In Prozent \\
\hline 209 & 25 & $11,96 \%$ \\
\hline
\end{tabular}

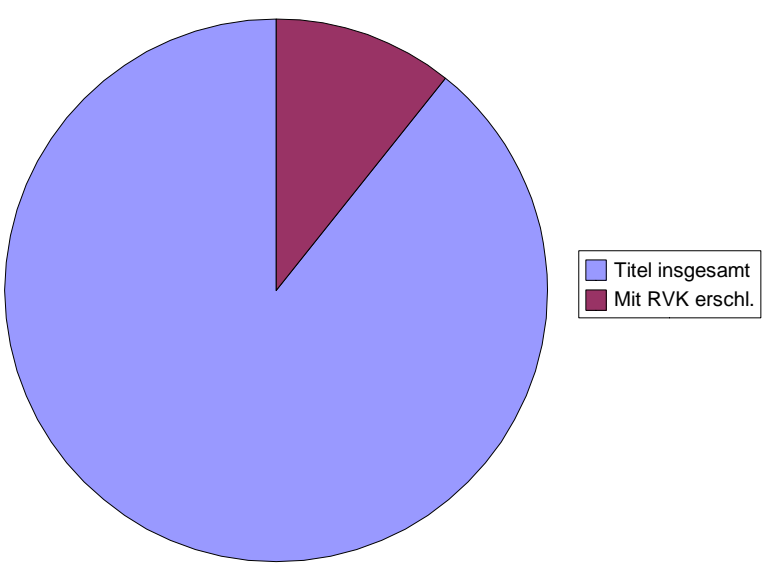

Von den 209 getesteten Alten Drucken sind im Dreiländerkatalog 25 mittels der RVK klassifikatorisch erschlossen. Dies sind 11,96 \% der Gesamtanzahl. 
5.2.3 Titel mit Schlagwort

\begin{tabular}{|c|c|c|}
\hline Titel insgesamt & Mit SW erschlossen & In Prozent \\
\hline 209 & 41 & $19,62 \%$ \\
\hline
\end{tabular}

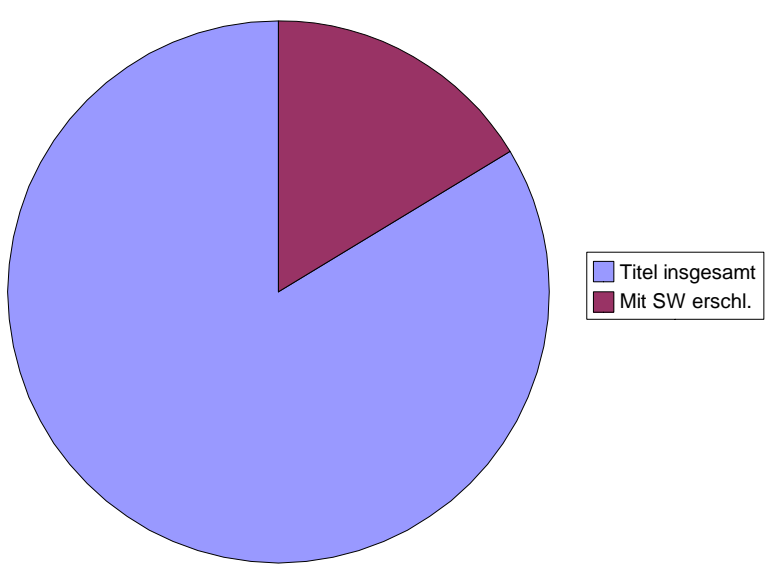

Von 209 Titeln sind 41 (19,62 \%) mit Hilfe eines oder mehrerer Schlagwörter unterschiedlicher Qualität erschlossen.

7 Titeldaten (3,35 \%) enthalten neben der RVK auch mindestens ein Schlagwort.

Dementsprechen sind im Dreiländerkatalog insgesamt 59 von 209 getesteten Titeln mit inhaltlichen Erschließungsdaten angereichert. Dies sind 28,23 \% der Gesamttitelmenge. 


\subsection{Karlsruher Virtueller Katalog}

Der Karlsruher Virtueller Katalog ${ }^{96}$ ist ein Meta-Katalog, über den sowohl in Katalogen in- und ausländischer Bibliotheken als auch in deutschen Buchhandelsverzeichnissen recherchiert werden kann. Mit einer Abfrage ist es möglich, gleichzeitig in zahlreichen verschiedenen Katalogen mit einem Datenbestand von insgesamt über 500 Millionen Titeln zu recherchieren. Dabei verfügt der KVK nicht über eine eigene Datenbank, sondern leitet die Suchanfrage an die jeweiligen, vom Nutzer zuvor ausgewählten Kataloge weiter.

\subsubsection{Bereits erschlossen}

Der recherchierte Titel ist bereits im OPAC der UB Augsburg entweder per Regensburger Verbundklassifikation (RVK) oder per Schlagwort (SW) erschlossen. Eine weitere Bearbeitung hinsichtlich der Sacherschließung ist somit nicht nötig.

\begin{tabular}{|c|c|c|c|}
\hline Titel insgesamt & per RVK & per SW & \\
\hline 209 & 14 & 5 & Titel \\
\hline & $6,70 \%$ & $2,39 \%$ & In Prozent \\
\hline
\end{tabular}

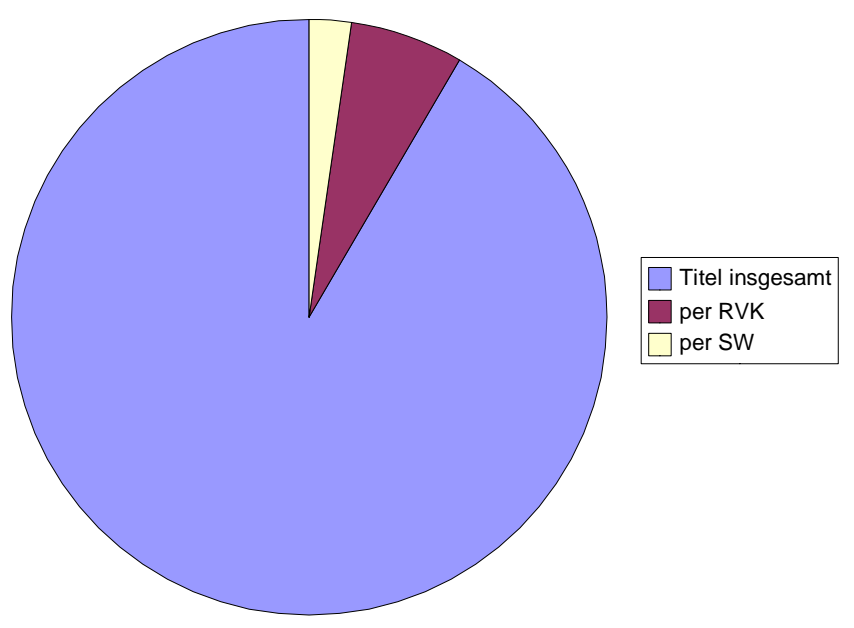

Von insgesamt 209 Titeln sind 14 (6,70\%) anhand der RVK und 5 (2,39 \%) mit Hilfe eines oder mehrerer Schlagwörter erschlossen. 3 Titel (1,44 \%) sind zusätzlich zur RVK auch mit mindestens einem Schlagwort versehen worden. Im Gesamten tragen demnach 16 Titel inhaltserschließende Daten. Das sind 7,66 \% der Gesamtmenge.

96 Zu finden unter KVK Karlsruher Virtueller Katalog (2008). Universitätsbibliothek Karlsruhe. Online verfügbar unter http://www.ubka.uni-karlsruhe.de/kvk.html, zuletzt aktualisiert am 29.01.2008, zuletzt geprüft am 17.02.2008. 


\subsubsection{Titel genügt}

Der Titel des Werkes reicht zum thematischen Auffinden des Druckes aus. Es ist keine weitere inhaltliche Erschließung mehr unbedingt notwendig. Der Druck kann entweder so belassen oder es können einzelne Wörter des Titels als Stichwort bzw. als freies Schlagwort behandelt werden. Eventuell ist aufgrund des gegebenen Titels eine unkomplizierte und schnelle Verschlagwortung nach den RSWK möglich.

Beispiel:

Canzler, Johann Georg: Nachrichten zur genauern Kenntniß der Geschichte, Staatsverwaltung und ökonomischen Verfassung des Königreichs Schweden. 1778

Bei diesem Werk reicht der Titel zum Auffinden des Buches aus, da er bereits im Haupttitel die Wörter Geschichte und Schweden trägt. Recherchiert man nun im OPAC in allen Feldern oder auch nur im Titelfeld nach „Geschichte / Schweden“, wird man diesen Titel als Ergebnis angezeigt bekommen, auch ohne zusätzliche inhaltliche Erschließung. Wollte man inn dennoch sachlich erschließen, wäre dies relativ schnell erledigt. Geschichte und Schweden als freie Schlagwörter oder Stichwörter. Jedoch wäre, da die beiden Begriffe ja schon normierte Schlagwörter darstellen, auch in vertretbarer Zeit eine Schlagwortkette nach den RSWK angelegt oder eine Klassifikation z.B. nach der RVK durchgeführt:

Schlagwortkette: Schweden ; Geschichte Anfänge $-1778^{97}$

97 Wobei hier wie auch bei allen anderen beispielhaften Stich- und Schlagwortansetzungen berücksichtigt werden muss, dass die jeweilige Ansetzung nur als Beispiel dient und weder Anspruch auf absolute Richtigkeit noch Anspruch auf Vollständigkeit bezüglich der einzelnen Inhalte des Titels erhebt.

Da bei einer solchen Vorgehensweise der Titel nur über die Begriffe Schweden und Geschichte aufzufinden ist, wäre es noch möglich, eine Software zur Automatischen ErschlieBung darauf anzuwenden, wie z.B. MILOS, die aufgrund von integrierten Wörterbüchern automatisch Synonyme der verwendeten Begriffe generiert. Dies gilt auch für alle weiteren Betrachtungen. 


\begin{tabular}{|c|c|c|}
\hline Titel insgesamt & Titel ist ausreichend & \\
\hline 209 & 12 & Titel \\
\hline & $5,74 \%$ & In Prozent \\
\hline
\end{tabular}

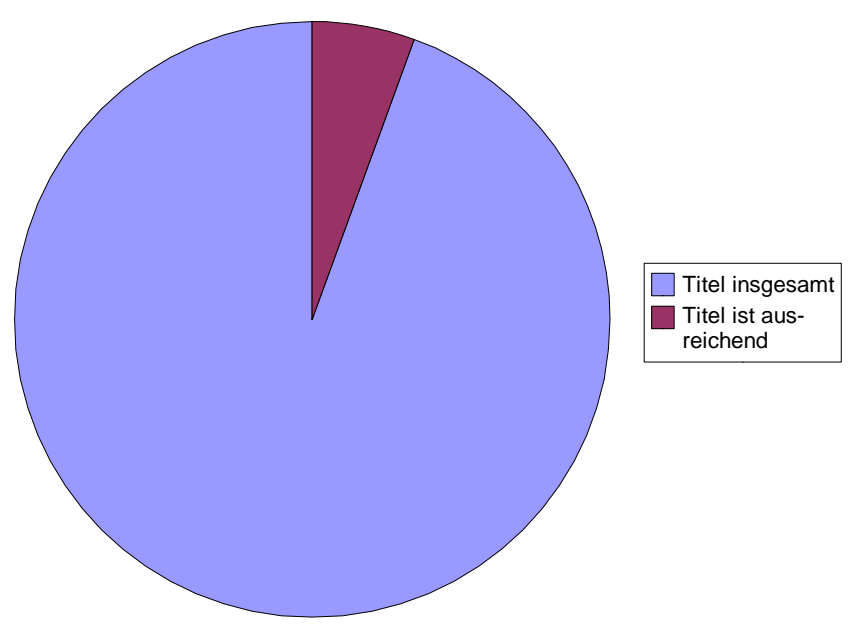

Zwölfmal, also bei 5,74 \% des untersuchten Bestandes, ist eine nähere Erschließung nicht zwingend notwendig, da der Titel des Werkes bereits klar Auskunft über das Thema erteilt. 


\subsection{3 Übersetzung genügt}

Evtl. genügt eine Übersetzung des Titels bzw. einzelner Wörter des Titels aus dem meist Französischen/Lateinischen, um diesen thematisch auffindbar zu machen, wobei dies natürlich nicht immer mit absoluter Sicherheit gesagt werden kann. Bei Französisch oder Latein (und natürlich auch Englisch, wobei alte Drucke im deutschsprachigen Raum nur relativ selten in dieser Sprache verfasst sind), kann man ein, wenn auch nur geringfügiges Verstehen und die damit verbundene Möglichkeit der zügigen Übersetzung voraussetzen. Für skandinavische Sprachen wie Dänisch oder Schwedisch gilt dies jedoch nicht. Indes sind auch diese Sprachen nur sehr selten anzutreffen; auch innerhalb der Thematik "Geschichte der skandinavischen Reiche“ waren nur sehr wenige Drucke in einer nordischen Sprache. Die einzelnen übersetzten Titelwörter könnten so behandelt werden wie unter Punkt 5.3.2. erläutert, also als Stich- oder freie Schlagwörter, eventuell auch als normierte Schlagwörter.

Beispiel:

Pufendorf, Samuel von: Histoire de Suède avant et depuis la fondation de la monarchie. 1743. 3 Bände

Stichworte: Geschichte ; Schweden

Schlagwortkette: Schweden ; Geschichte ; Anfänge -1743

\begin{tabular}{|c|c|c|}
\hline Titel insgesamt & $\begin{array}{c}\text { Übersetzung ist ausrei- } \\
\text { chend }\end{array}$ \\
\hline 209 & 17 & Titel \\
\hline & $8,13 \%$ & In Prozent \\
\hline
\end{tabular}

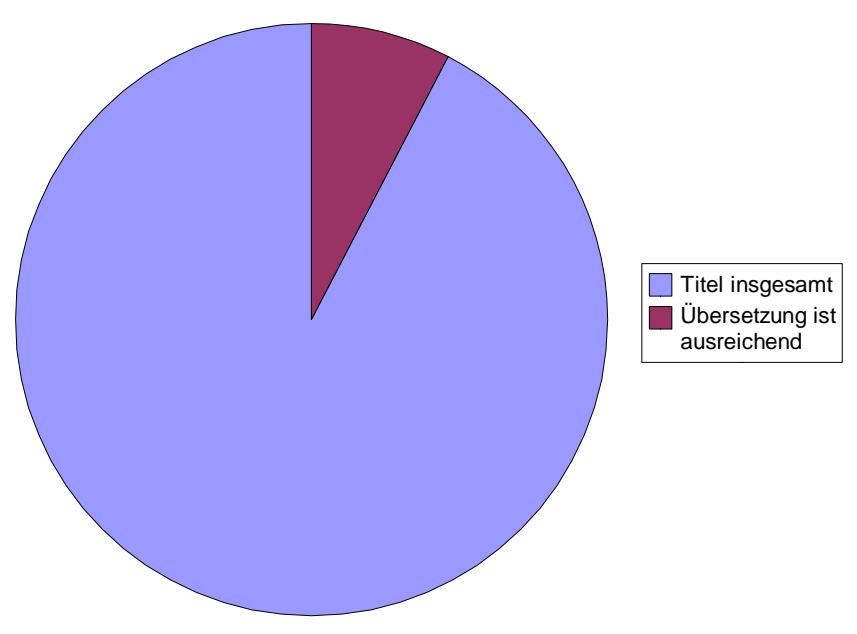


Bei 17 Titeln, 8,13\% des Bestandes, ist es möglich, durch eine schnelle Übersetzung des Titels bzw. einzelner Wörter des Titels ins Deutsche eine zügige Erschließung in Form von Stich- oder Schlagwörtern zu ermöglichen.

\subsubsection{Ortographische Anpassung genügt}

Evtl. genügt eine Anpassung des Titels bzw. einzelner Wörter des Titels aus einer älteren deutschen Schreibweise in die moderne Form, um den Titel thematisch auffindbar zu machen. Diese orthographisch angepassten Titelwörter können wiederum als Stichwörter oder als Schlagwörter verwendet werden und dementsprechend bei der Erschließung in ein dafür vorgesehenes Feld eingetragen werden.

Beispiel:

Cize, Emmanuel de:

Curieuse Nachricht von denen zweyen in England einander hefftig zuwider seyenden Partheyen derer Whigs und Torys worin derselben Ursprung, Wachsthum, auch listige und zum Theil gefährliche Unternehmungen... beschrieben werden. 1718.

Stichworte: England; Parteien

Schlagwortkette: Großbritannien; Parteiensystem

\begin{tabular}{|c|c|c|}
\hline Titel insgesamt & $\begin{array}{c}\text { Anpassung ist ausrei- } \\
\text { chend }\end{array}$ \\
\hline 209 & 8 & Titel \\
\hline & $3,83 \%$ & In Prozent \\
\hline
\end{tabular}

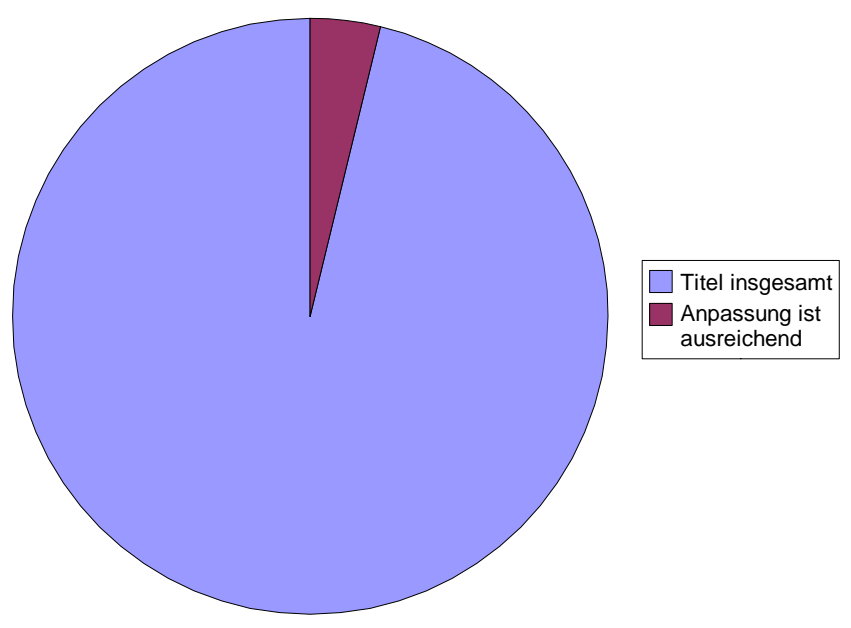

Bei insgesamt 8 Titeln (3,83 \%) genügt eine Anpassung der Orthographie. 


\subsubsection{Titel zu speziell}

Natürlich ist auch bei Historischen Beständen nicht jedes Werk gleich bedeutsam. So kann es durchaus sein, dass ein Werk unter pragmatischen Gesichtspunkten eventuell zu unwichtig erscheint, um unbedingt erschlossen werden zu müssen. Dabei handelt es sich meist um kleine Drucke von wenigen Seiten, deren Themen sehr speziell und daher sowohl für die Allgemeinheit als auch für die allermeisten Wissenschaftler eher uninteressant sind. Wobei die Entscheidung, ob etwas weniger wichtig ist, natürlich nur sehr schwer und sehr subjektiv gefällt werden und durchaus Widerspruch hervorrufen kann.

Beispiel:

Ausführliche Beschreibung der solennen Einbegleitung zur öffentlichen kayserl. Audienz Ihrer hoch-gräflichen Excell. des Herrn General-Feld-Marschalls, des H. R. Reichs Grafen von Flemming geschehen in Wien am 13. Augusti Anno 1719. 1719.

\begin{tabular}{|c|c|c|}
\hline Titel insgesamt & $\begin{array}{c}\text { Erschließung nicht not- } \\
\text { wendig }\end{array}$ & \\
\hline 209 & 16 & Titel \\
\hline & $7,66 \%$ & In Prozent \\
\hline
\end{tabular}

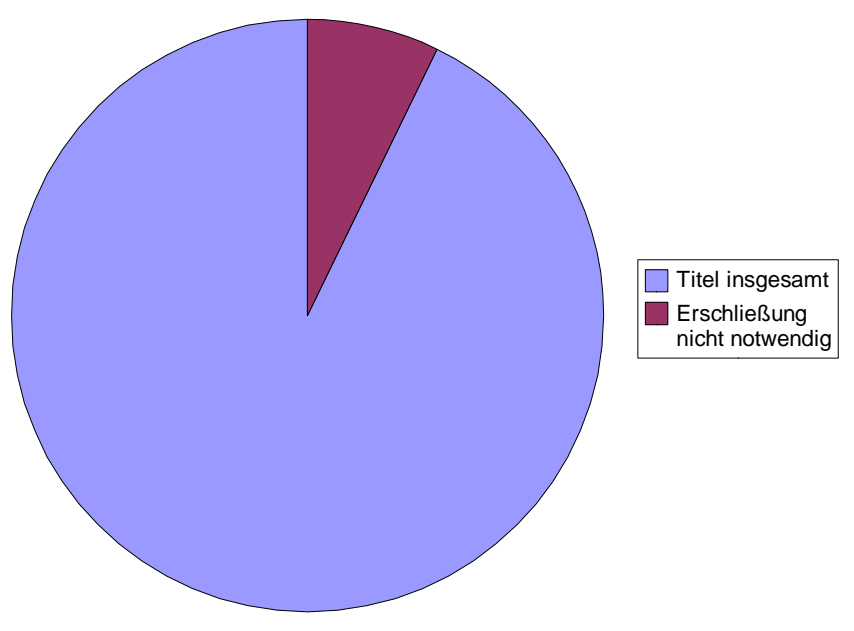

Bei insgesamt 16 von 209 Titeln (7,66 \%) kann man feststellen, dass deren Inhalt sehr speziell ist und aus diesem Grund auf eine aufwändige Erschließung eventuell verzichtet werden kann. 


\subsubsection{Schnelle Verschlagwortung}

Auch Alte Drucke besitzen Sachtitel, die deutlich Auskunft darüber geben, welcher Inhalt in den jeweiligen Werken thematisiert wird. Bei solchen Titeln kann relativ schnell eine Schlagwortkette oder eine Notation angelegt werden, ohne dass man dabei auf größere, zeitraubende Probleme stösst.

Beispiel:

Mauvillon, Eléazar:

Geschichte Gustav Adolphs, Königs von Schweden. 1775.

Schlagwortkette: Gustav Adolf <Schweden, König, II.> ; Biographie

\begin{tabular}{|c|c|c|}
\hline Titel insgesamt & $\begin{array}{c}\text { Schlagwort schnell ange- } \\
\text { legt }\end{array}$ & \\
\hline 209 & 51 & Titel \\
\hline & $24,40 \%$ & In Prozent \\
\hline
\end{tabular}

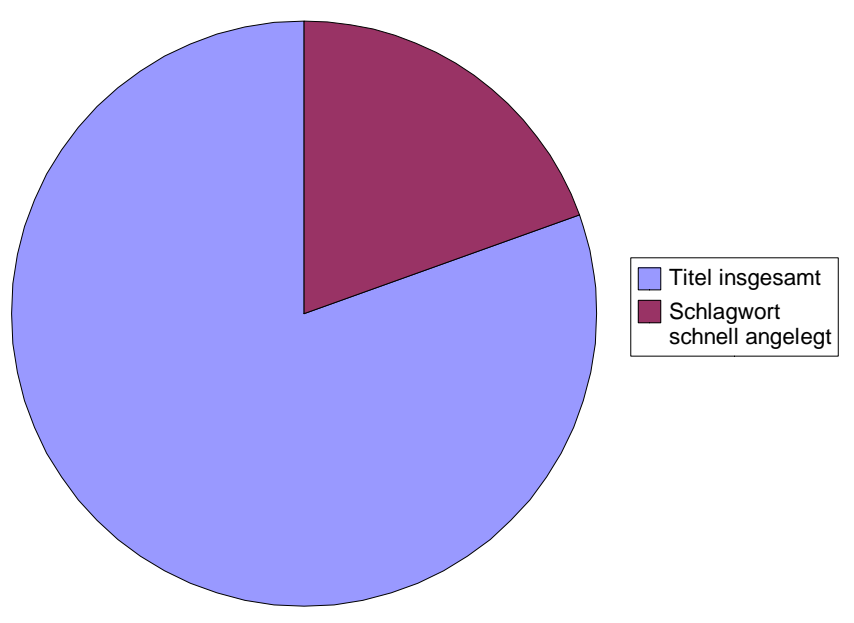

Bei beinahe einem Viertel der überprüften Titel $(24,40 \%)$ ist durch die Aussagekraft des Titels eine Verschlagwortung bzw. eine Klassifizierung in vertretbarer Zeit durchzuführen. 


\subsubsection{Nur in der UB Augsburg vorhanden}

Der Titel konnte bei der Überprüfung nur in der UB Augsburg nachgewiesen werden. Dies weist auf eine relative Seltenheit der betreffenden Drucke hin. Eine Übernahme von Erschließungsdaten aus anderen Bibliotheken oder Verbünden ist somit nicht möglich.

\begin{tabular}{|c|c|c|}
\hline Titel insgesamt & $\begin{array}{c}\text { Nur in der UBA vorhan- } \\
\text { den }\end{array}$ & \\
\hline 209 & 8 & Titel \\
\hline & $3,83 \%$ & In Prozent \\
\hline
\end{tabular}

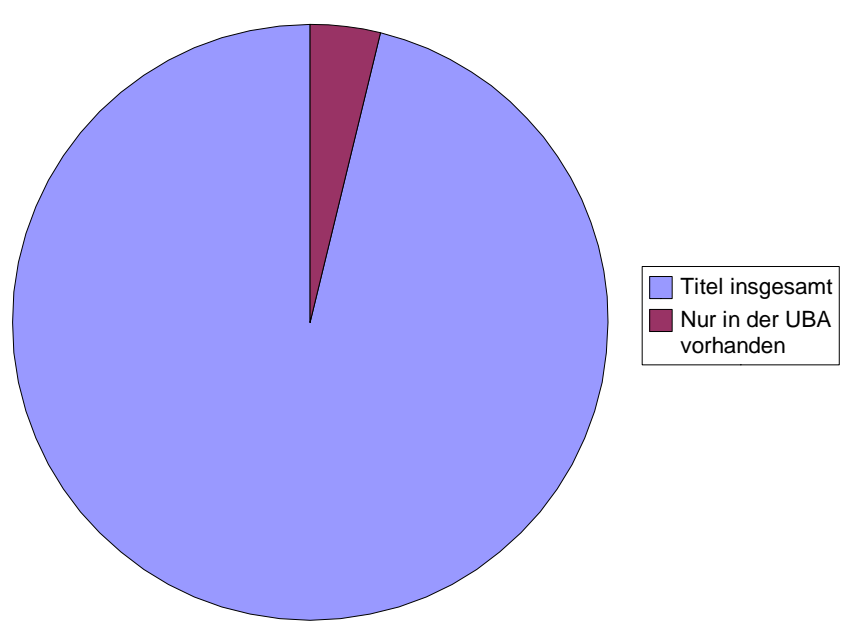

Von 209 Titeln sind somit acht (3,83 \%) lediglich in der UB Augsburg vorhanden. 


\subsubsection{Erschließung per Autopsie}

Muss man, um es inhaltlich erschließen zu können, das Buch zur Hand nehmen? Oder ist es möglich, nur anhand der Titeldaten eine Sacherschließung durchzuführen?

Eine Erschließung ohne Autopsie bringt den großen Vorteil der Zeiteinsparung mit sich. Man kann ohne Vorlage des Buches anhand von formalen Katalogdaten die ErschlieBung vornehmen. Es ist z.B. nicht nötig, das Buch aus dem Magazin hervorzuholen und es einer genauen Betrachtung zu unterziehen. Bei einer Erschließung anhand der Titeldaten verringert sich der Aufwand erheblich.

Natürlich bringt eine solche Vorgehensweise es mit sich, dass das Risiko einer falschen oder fehlerhaften Erschließung wesentlich höher liegt als bei einer autoptischen Erschließung. Auch die Erschließungstiefe kann darunter leiden. Der Zeitvorteil jedoch ist erheblich. Natürlich hängen die Möglichkeiten dieser Erschließungsart auch von den jeweiligen thematischen Kenntnissen der Person ab, welche die Erschließung durchführt.

Auf Autopsie kann z.B. bei folgendem Titel verzichtet werden:

Beer, Johann C.:

Der von Christi Geburt an, biß auf diese unsere Zeit, regierenden Könige in Schweden Leben, Regierung und Absterben aus der Bewehrtesten schwedischen Geschicht-Schreibern hervorgesucht u. Zsgetragen... 1673

Auf eine Autopsie kann nicht verzichtet werden:

Nettelbladt, Christian von:

Schwedische Bibliothek. 1728

Ob auf eine Autopsie verzichtet werden kann, kann nicht verbindlich entschieden werden:

Treuer, Gotthilf:

Kurtze Beschreibung der heidnischen Todten-Töpffe in welchen die Heiden ihrer verbrannten Todten überbliebene Gebein und Aschen aufgehoben unter der Erde beygesetzet... 1688 


\begin{tabular}{|c|c|c|c|}
\hline Titel insgesamt: & Ohne Autopsie & Mit Autopsie & Neutral \\
\hline 209 & 115 & 58 & 36 \\
\hline In Prozent & $55,02 \%$ & $27,75 \%$ & $17,22 \%$ \\
\hline
\end{tabular}

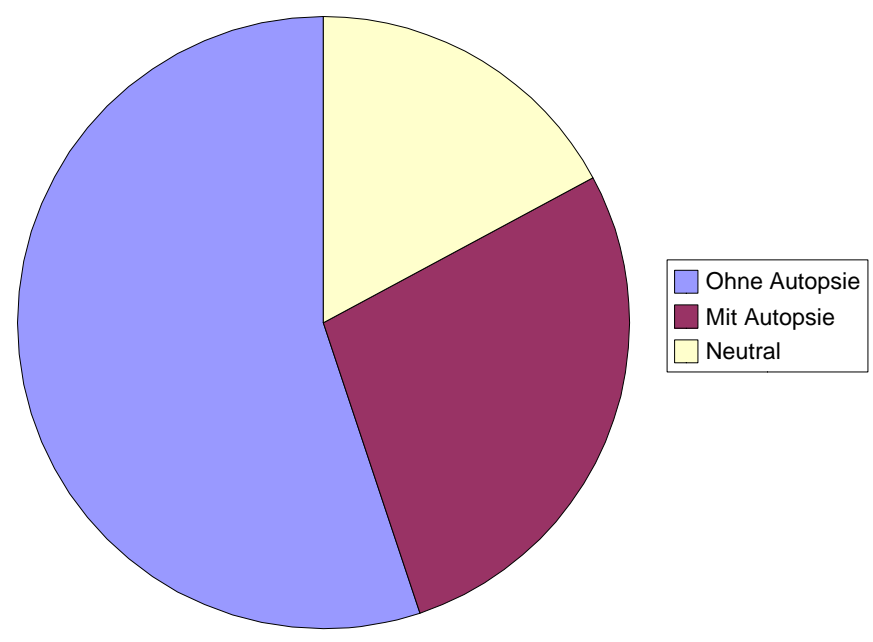

Demzufolge besteht bei 115 Titeln die Möglichkeit, sie ohne Autopsie zu erschließen. Dies sind 55,02 \%, also über die Hälfte der getesteten Werke. Nur autoptisch können 58 Titel $(27,7 \%)$ inhaltlich erschlossen werden. Bei 36 Titeln (17,22 \%) ist es unklar, ob eine Erschließung nur anhand der Titeldaten, ohne Autopsie, durchgeführt werden kann.

Abschließend muss gesagt werden, dass bei der Bearbeitung der Bestandsgruppe IV.18 (Geschichte der skandinavischen Reiche) auffiel, dass die Aussage des Titels häufig sehr konkret und deutlich auf die behandelte Thematik bezogen war. Ob dies generell bei sämtlichen Thematiken von alten Drucken der Fall ist, kann bezweifelt werden. Jedoch fiel bei einer Gegenstichprobe, die anhand von 50 Titeln aus der Systematikgruppe VI. (Pädagogik) durchgeführt wurde, das Ergebnis überaschenderweise sehr ähnlich aus. So könnten 28 Titel (56 \%) ohne Autopsie erschlossen werden, bei 14 (28 $\%$ ) ist dies nicht möglich und bei 8 Titeln (16\%) fand keine Festlegung statt, wobei es auch in diesen Fällen nicht immer eindeutig zu entscheiden war. 


\subsubsection{Betrachtungen einzelner Verbünde und Bibliotheken}

\subsubsection{Getestete Kataloge}

Folgende Bibliotheken und Verbünde wurden in den Test miteinbezogen:

Gemeinsamer Bibliotheksverbund $\quad$ GBV

Hochschulbibliothekszentrum des Landes Nordrhein-Westfalen $\quad$ HBZ

Hessisches BibliotheksInformationsSystem HeBIS

Kooperativer Bibliotheksverbund Berlin-Brandenburg KOBV

Österreichische Nationalbibliothek Katalog 1501-1929 ÖNB

Staatsbibliothek zu Berlin $\quad$ StaBi

Südwestdeutscher Bibliotheksverbund SWB

Insgesamt wurden 209 Titel in 276 Bänden ausgewertet. 
5.3.9.2 Vorkommen der Titel

Insgesamt

GBV

SWB

StaBi

HBZ

ÖNB

HeBIS

KOBV
209 Titel

186

151

147

85

62

37

20
In Prozent:

$89,00 \%$

$72,25 \%$

$70,33 \%$

$40,67 \%$

$29,67 \%$

$17,70 \%$

$9,57 \%$

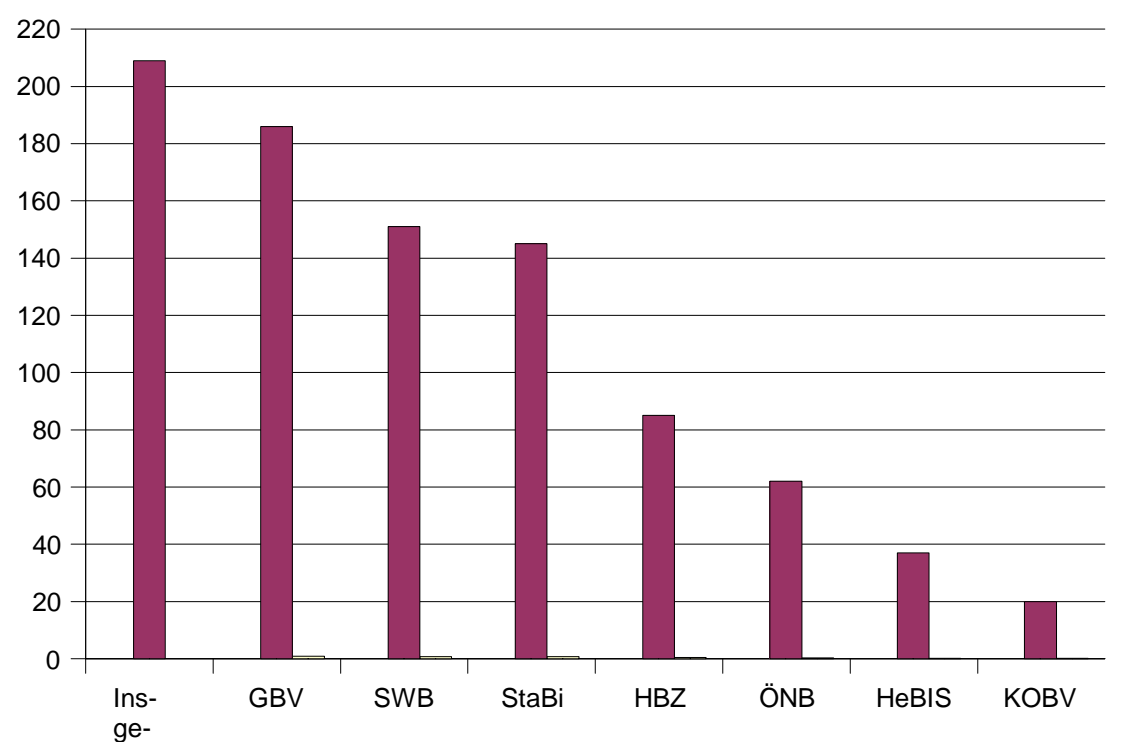




\subsubsection{GBV}

\begin{tabular}{|c|c|c|c|}
\hline Titel insg. & Im GBV vorhanden & Mit SW Erschlossen & SW Standardisiert \\
\hline 209 & 186 & 67 & 33 \\
\hline
\end{tabular}

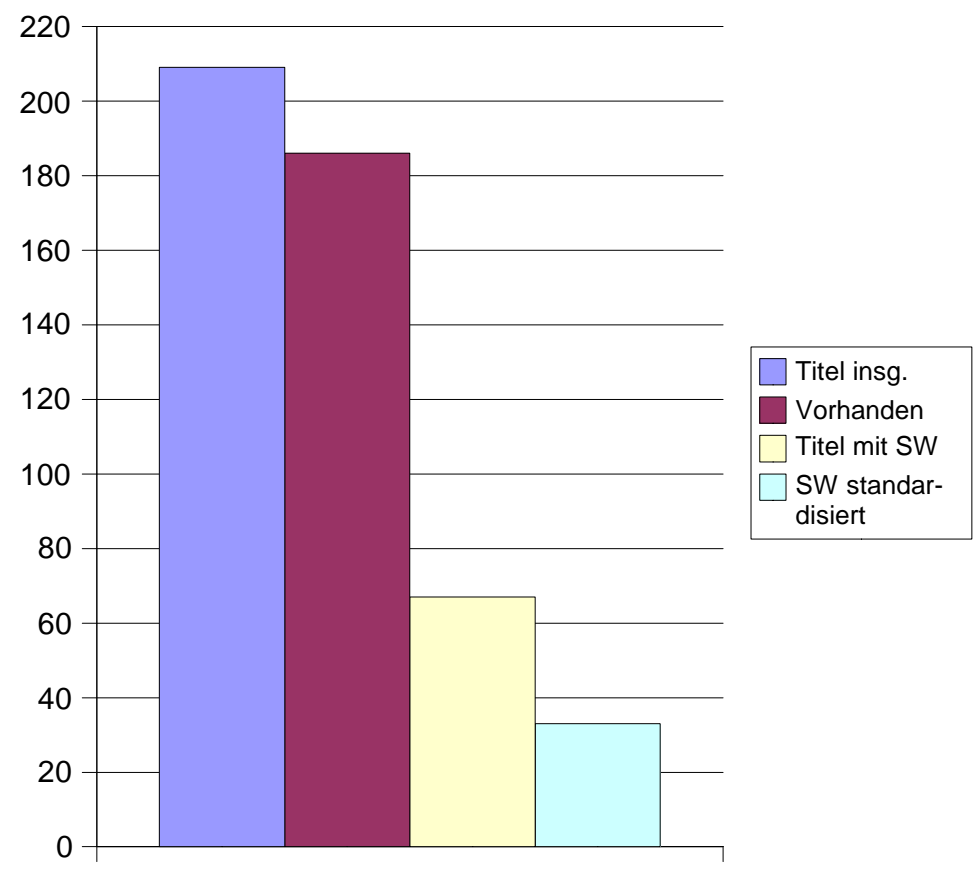

\begin{tabular}{|c|c|c|}
\hline Von 209 Titeln sind & Vorhanden & Nicht vorhanden \\
\hline $\begin{array}{c}\text { Von diesen } 89,00 \% \text { sind } \\
\text { per SW }\end{array}$ & $89,00 \%$ & $11,00 \%$ \\
\hline & Erschlossen & Nicht erschlossen \\
\hline
\end{tabular}
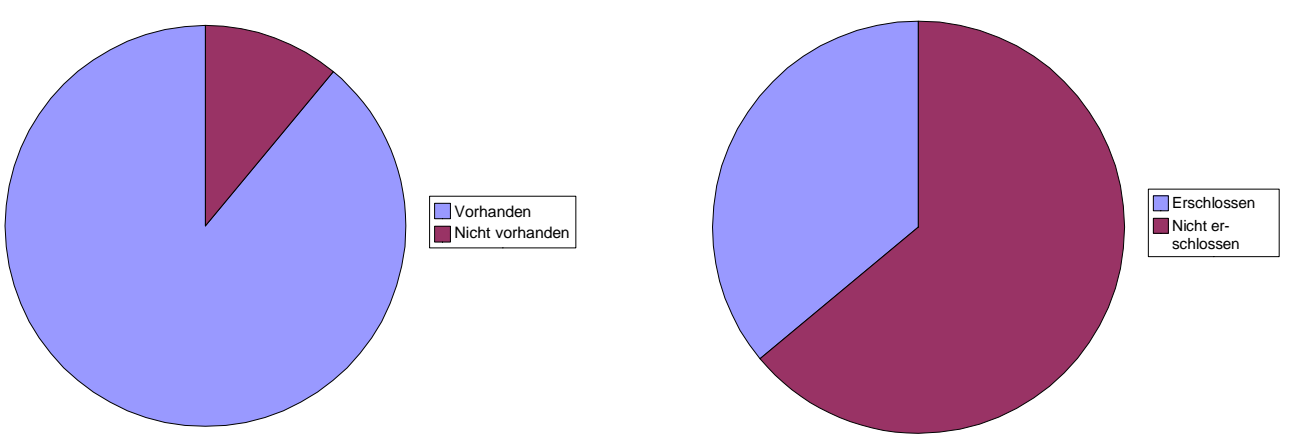


\begin{tabular}{|c|c|c|}
\hline Von diesen 36,02 \% sind & Übernehmbar & Nicht standardisiert \\
\hline & $49,25 \%$ & $50,75 \%$ \\
\hline $\begin{array}{c}\text { Insgesamt bedeutet dies, } \\
\text { von 209 Titeln sind }\end{array}$ & Erschlossen & Nicht erschlossen \\
\hline & $32,06 \%$ & $67,94 \%$ \\
\hline
\end{tabular}
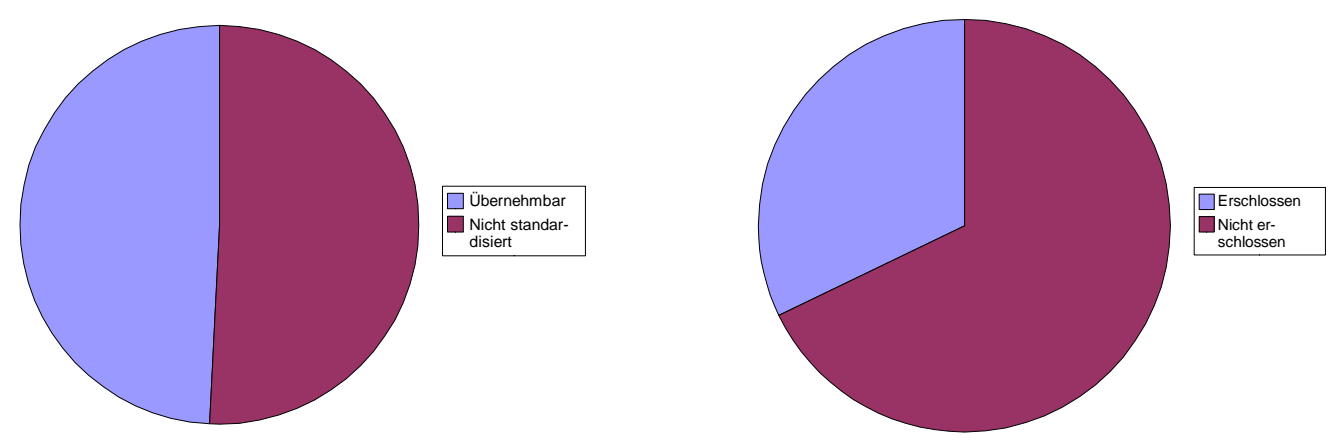

Übernehmbare Erschließungsdaten bezogen auf die Gesamtmenge:

\begin{tabular}{|c|c|}
\hline Standardisiert & $\begin{array}{c}\text { Nicht verschlagwortet bzw. nicht } \\
\text { standardisiert }\end{array}$ \\
\hline $15,79 \%$ & $84,21 \%$ \\
\hline
\end{tabular}

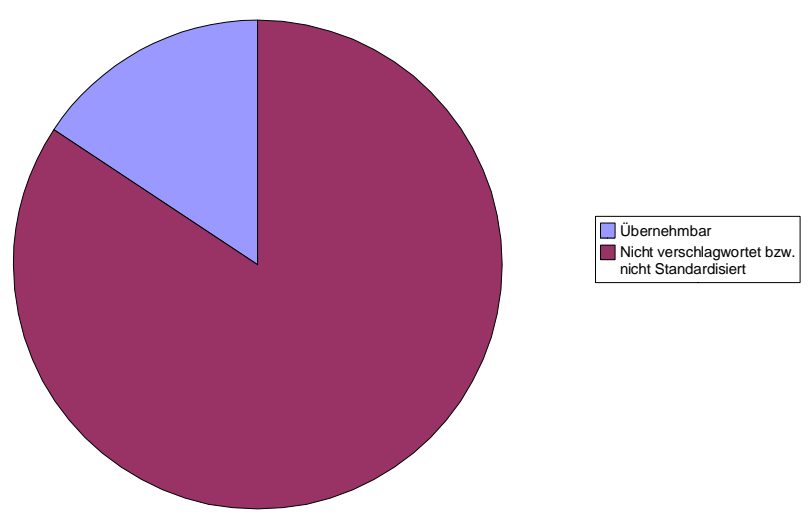

Im Gemeinsamen Bibliotheksverbund sind von den getesteten 209 Titeln 186 vorhanden $(89,00 \%)$ und somit 23 nicht vorhanden (11,00\%). Von den vorhandenen $186 \mathrm{Ti}$ teln sind $67(36,02 \%)$ mit einem oder mehreren Schlagwörtern erschlossen. Von den vorhandenen Titeln nicht per Schlagwort erschlossen sind somit 119 Titel, 63,98 \%. 
Von diesen 67 durch Schlagwörter erschlossenen Titeln ist diese Verschlagwortung bei 33 Titeln (49,25\%) in solcher Qualität, d.h. standardisiert, dass die Erschließungsdaten von anderen Verbünden übernehmbar sind. Bei 34 der 67 verschlagworteten Titeln ist dies nicht der Fall (50,75\%). Dies schließt u.a., ebenso wie bei den weiteren Verbünden und Bibliotheken, nicht normierte Schlagwörter, Gattungsbegriffe, Library of Congress Subject Headings und die Hausregeln der ÖNB mit ein. Die innerhalb des GBV mit Gattungsbegriffen erschlossenen Titel werden nachfolgend separat verzeichnet.

Insgesamt bedeutet dies, dass von den 209 überprüften Titeln 32,06 \% inhaltlich erschlossen sind (67 Titel). Sachlich nicht erschlossen sind 67,94\% (142 Titel).

Alles in allem sind 33 der 209 getesteten Titel in solcher Qualität per Schlagwort erschlossen, dass diese Erschließungsdaten von anderen Verbünden übernommen werden können. Dies sind 15,79 \% der Gesamtmenge.

Zudem sind im GBV bestimmte Titel per Gattungsbegriff und Basisklassifikation erschlossen. Dies hängt mit der Mitgliedschaft der SUB Göttingen in der Arbeitsgemeinschaft Sammlung Deutscher Drucke und der darin getragenen Verantwortung für das Zeitsegment des 18. Jahrhunderts ${ }^{98}$ sowie mit der Arbeitsgemeinschaft Alte Drucke im GBV zusammen. Zur Vergabe der Gattungsbegriffe wird innerhalb der Arbeitsgemeinschaft die AAD-Liste von Gattungs- und Sachbegriffen ${ }^{99}$ herangezogen.

Bei den ermittelten Zahlen muss jedoch beachtet werden, dass eine Verschlagwortung per RSWK nicht die zusätzliche Anwendung eines Gattungsbegriffes oder der Basisklassifikation ausschließt.

98 Sieh Kap. 4.3

99 AAD Gattungs- und Sachbegriffe (2005) 


\section{Gattungsbegriffe}

\begin{tabular}{|c|c|c|}
\hline Titel insgesamt & $\begin{array}{c}\text { Mit Gattungsbegriff er- } \\
\text { schlossen }\end{array}$ & In Prozent \\
\hline 209 & 33 & $15,79 \%$ \\
\hline
\end{tabular}

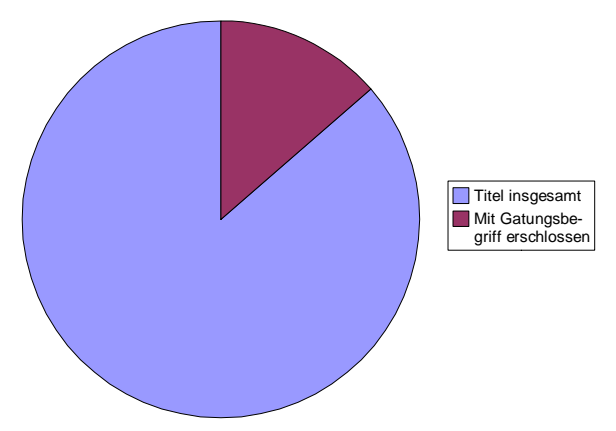

33 der 209 Titel (15,79\%) sind mit einem oder mehreren Gattungsbegriffen versehen worden.

\section{Basisklassifikation}

\begin{tabular}{|c|c|c|}
\hline Titel insgesamt & Mit BK erschlossen & In Prozent \\
\hline 209 & 39 & $18,66 \%$ \\
\hline
\end{tabular}

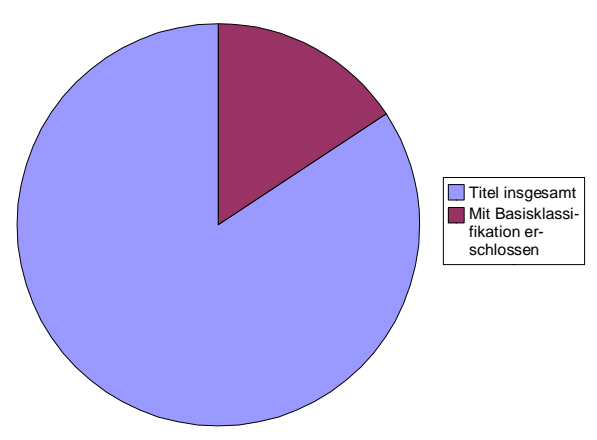

39 der überprüften 209 Titel (18,66 \%) wurden mit der Basisklassifikation klassifiziert. 6 Titel $(2,87 \%)$ sind sowohl mit einem oder mehreren Gattungsbegriffen als auch mit der Basisklassifikation erschlossen. 


\subsubsection{SWB}

\begin{tabular}{|c|c|c|c|}
\hline Titel insg. & Vorhanden & Erschlossen & Standardisiert \\
\hline 209 & 151 & 3 & 1 \\
\hline
\end{tabular}

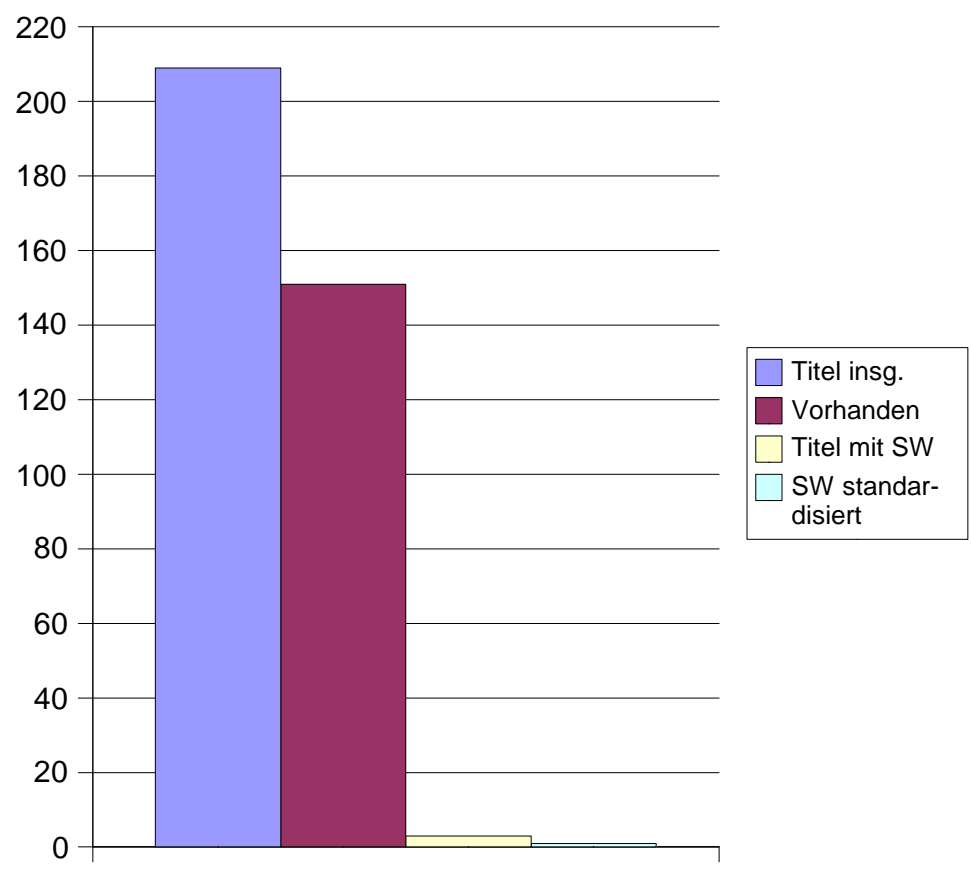

\begin{tabular}{|c|c|c|}
\hline Von 209 Titeln sind & Vorhanden & Nicht vorhanden \\
\hline & $72,25 \%$ & $27,75 \%$ \\
\hline $\begin{array}{c}\text { Von diesen } 72,25 \% \text { sind } \\
\text { per SW }\end{array}$ & Erschlossen & Nicht erschlossen \\
\hline & $1,99 \%$ & $98,01 \%$ \\
\hline
\end{tabular}
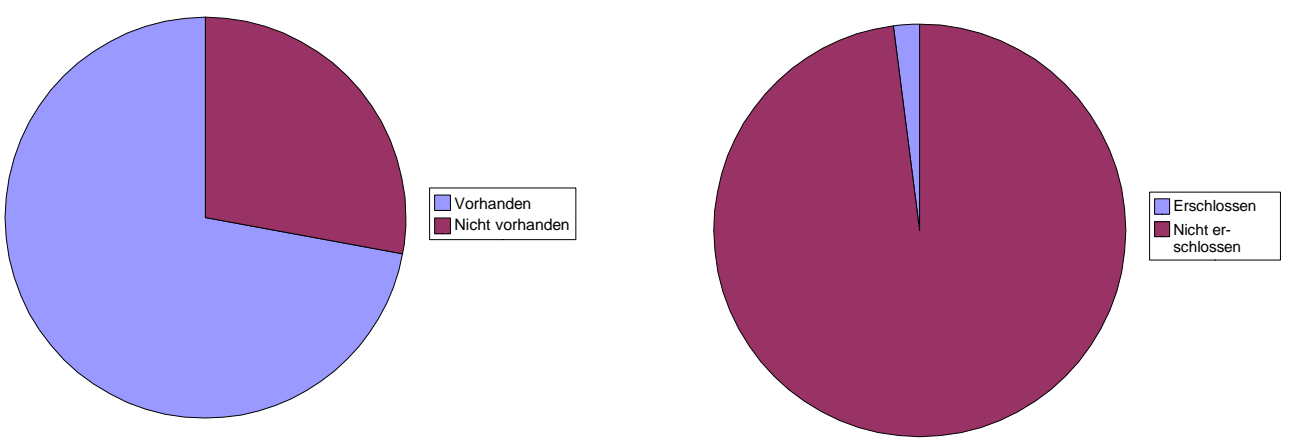


\begin{tabular}{|c|c|c|}
\hline Von diesen 1,99\% sind & Übernehmbar & Nicht standardisiert \\
\hline & $33,33 \%$ & $66,67 \%$ \\
\hline $\begin{array}{c}\text { Insgesamt bedeutet dies, } \\
\text { von 209 Titeln sind }\end{array}$ & Erschlossen & Nicht Erschlossen \\
\hline & $1,44 \%$ & $98,56 \%$ \\
\hline
\end{tabular}
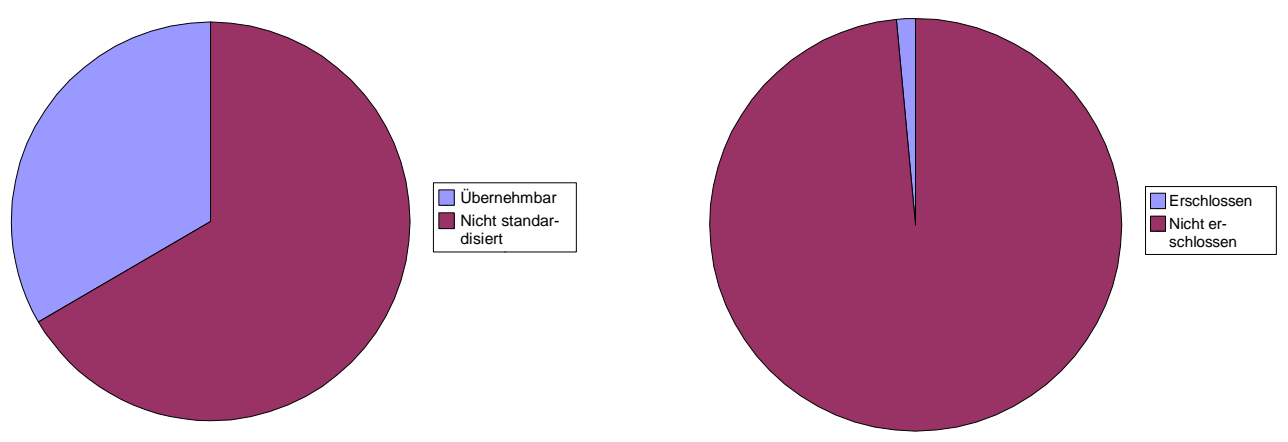

Übernehmbare Erschließungsdaten bezogen auf die Gesamtmenge:

\begin{tabular}{|c|c|}
\hline Standardisiert & $\begin{array}{c}\text { Nicht verschlagwortet bzw. nicht } \\
\text { standardisiert }\end{array}$ \\
\hline $0,48 \%$ & $99,52 \%$ \\
\hline
\end{tabular}

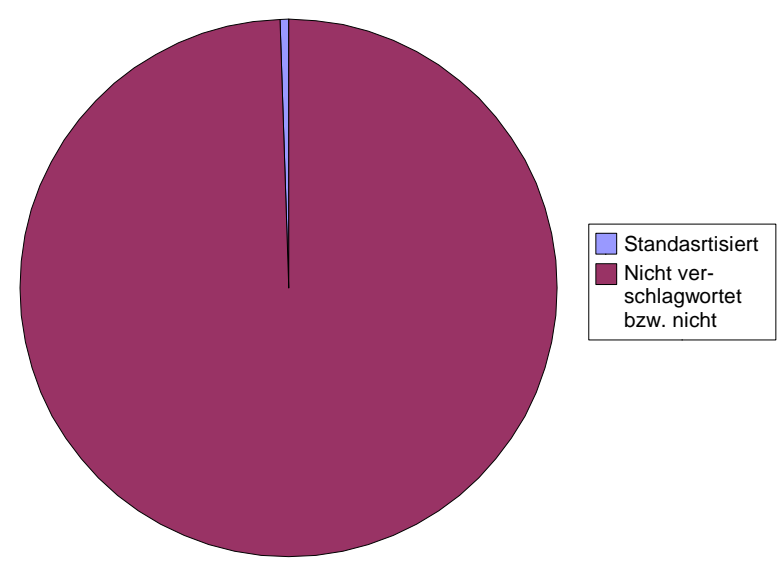

Somit sind beim SWB lediglich die Erschließungsdaten von einem der getesteten 209 Titel $(0,48 \%)$ für andere Verbünde übernehmbar. 
5.3.9.5 StaBi Berlin

\begin{tabular}{|c|c|c|c|}
\hline Titel insg. & Titel vorhanden & Titel mit SW & Titel standardisiert \\
\hline 209 & 147 & 13 & 8 \\
\hline
\end{tabular}

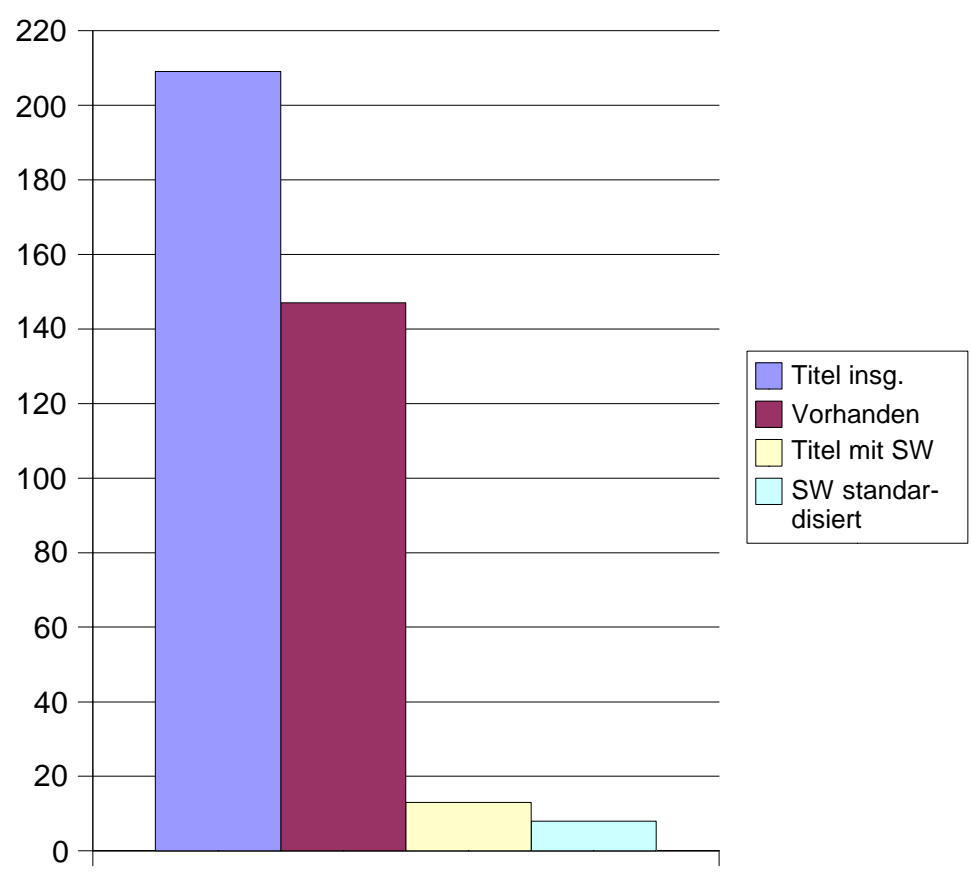

\begin{tabular}{|c|c|c|}
\hline Von 209 Titeln sind & Vorhanden & Nicht vorhanden \\
\hline & $70,33 \%$ & $29,67 \%$ \\
\hline $\begin{array}{c}\text { Von diesen } 70,33 \% \text { sind } \\
\text { per SW }\end{array}$ & Erschlossen & Nicht erschlossen \\
\hline & $8,84 \%$ & $91,16 \%$ \\
\hline
\end{tabular}
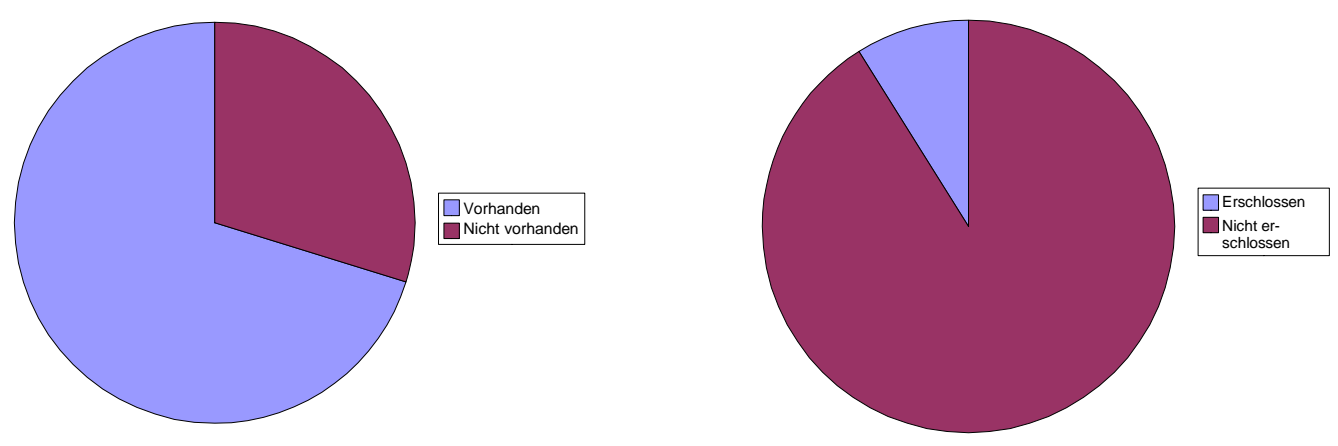


\begin{tabular}{|c|c|c|}
\hline Von diesen 8,84 \% sind & Übernehmbar & Nicht standardisiert \\
\hline & $61,54 \%$ & $38,46 \%$ \\
\hline $\begin{array}{c}\text { Insgesamt bedeutet dies, } \\
\text { von 209 Titeln sind }\end{array}$ & Erschlossen & Nicht erschlossen \\
\hline & $6,22 \%$ & $93,78 \%$ \\
\hline
\end{tabular}
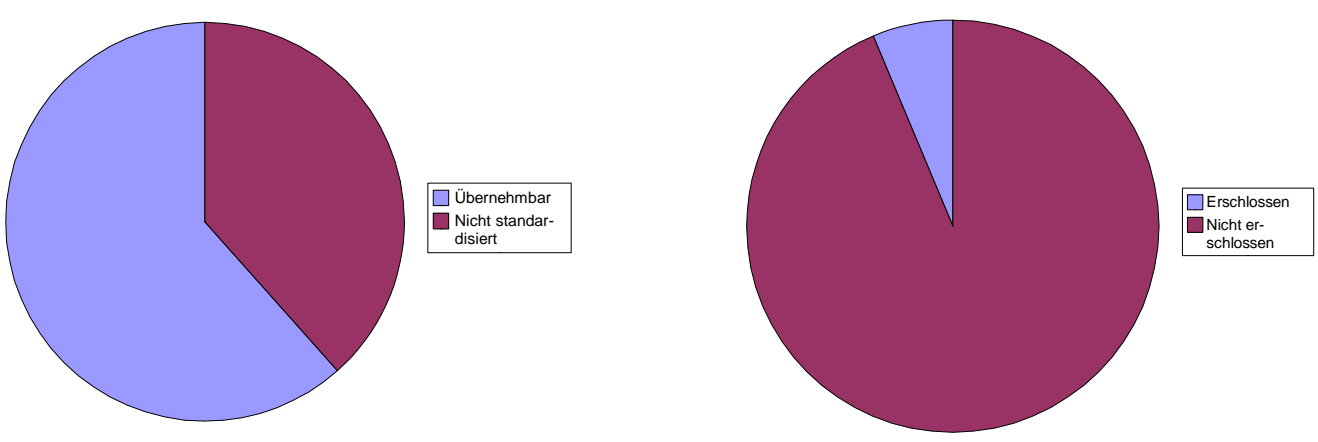

Übernehmbare Erschließungsdaten bezogen auf die Gesamtmenge:

\begin{tabular}{|c|c|}
\hline Standardisiert und übernehmbar & $\begin{array}{c}\text { Nicht verschlagwortet bzw. nicht standar- } \\
\text { disiert }\end{array}$ \\
\hline $3,83 \%$ & $96,17 \%$ \\
\hline
\end{tabular}

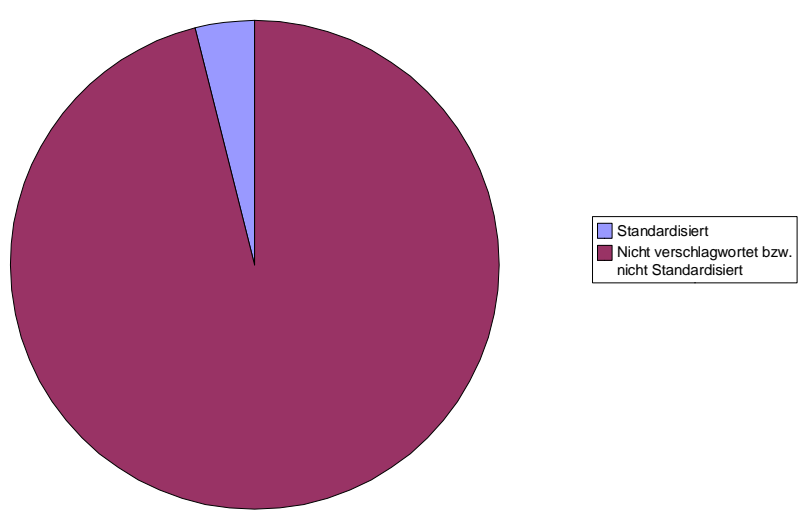

Auch bei der Staatsbibliothek zu Berlin sind nur bei 3,83 \% der Gesamtmenge (8 Titel) Erschließungsdaten für andere Bibliotheken und Verbünde nutzbar. 
5.3.9.6 HBZ

\begin{tabular}{|l|l|l|l|}
\hline Titel insg. & Vorhanden & Titel mit SW & SW standardisiert \\
\hline 209 & 85 & 2 & 2 \\
\hline
\end{tabular}

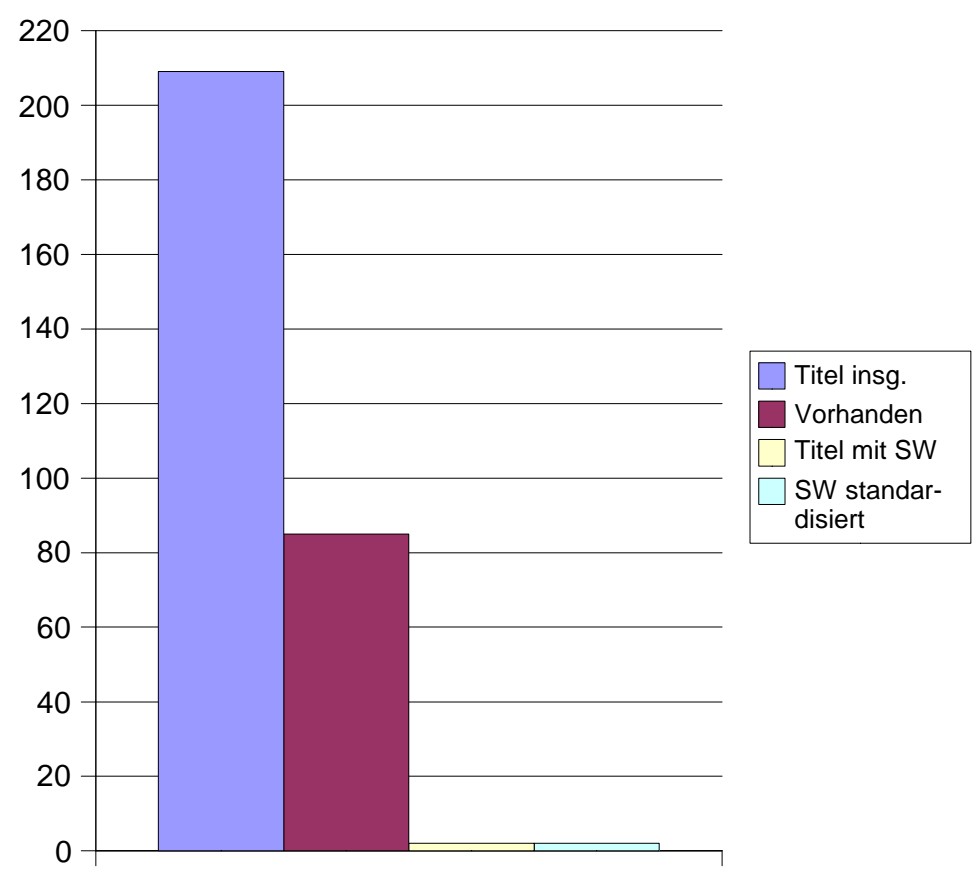

\begin{tabular}{|c|c|c|}
\hline Von 209 Titeln sind & Vorhanden & Nicht vorhanden \\
\hline $\begin{array}{c}\text { Von diesen 40,47 \% sind } \\
\text { per SW }\end{array}$ & $40,67 \%$ & $59,33 \%$ \\
\hline & Erschlossen & Nicht erschlossen \\
\hline
\end{tabular}
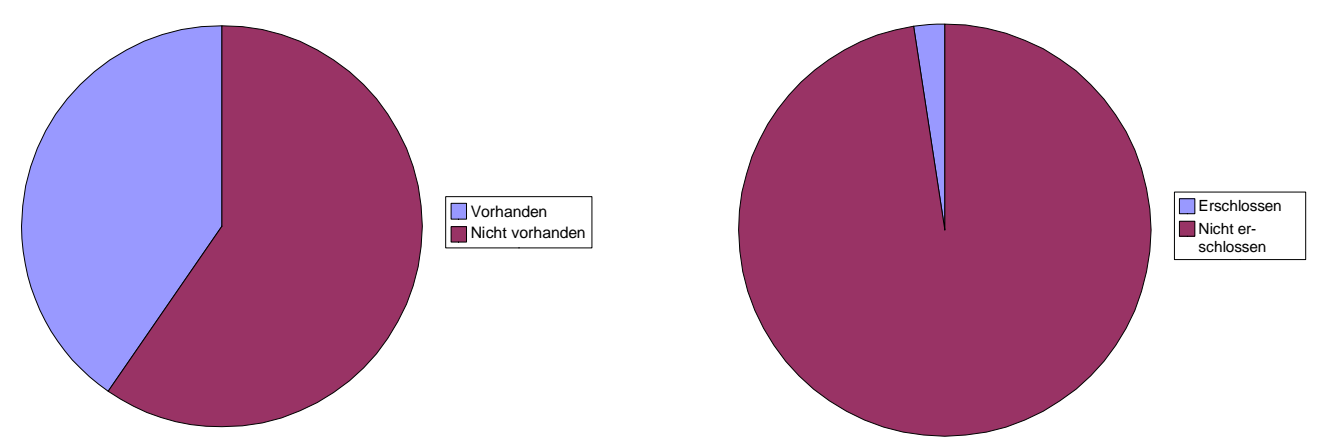


\begin{tabular}{|c|c|c|}
\hline Von diesen 2,35\% sind & Übernehmbar & Nicht standardisiert \\
\hline $\begin{array}{c}\text { Insgesamt bedeutet dies, } \\
\text { von 209 Titeln sind }\end{array}$ & Erschlossen & $0,00 \%$ \\
\hline & $0,96 \%$ & Nicht erschlossen \\
\hline
\end{tabular}
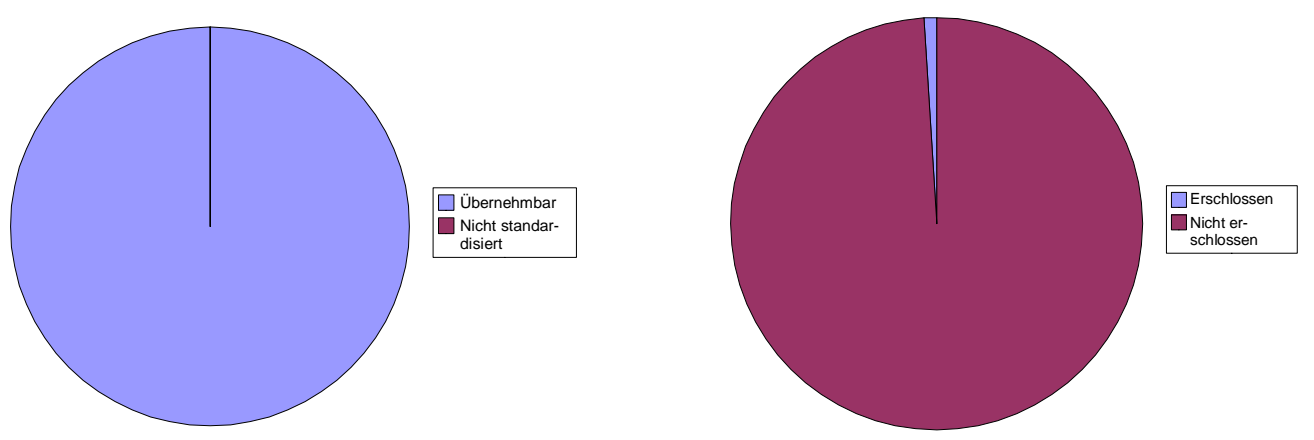

Übernehmbare Erschließungsdaten bezogen auf die Gesamtmenge:

\begin{tabular}{|c|c|}
\hline Standardisiert & $\begin{array}{c}\text { Nicht verschlagwortet bzw. nicht } \\
\text { standardisiert }\end{array}$ \\
\hline $0,96 \%$ & $99,04 \%$ \\
\hline
\end{tabular}

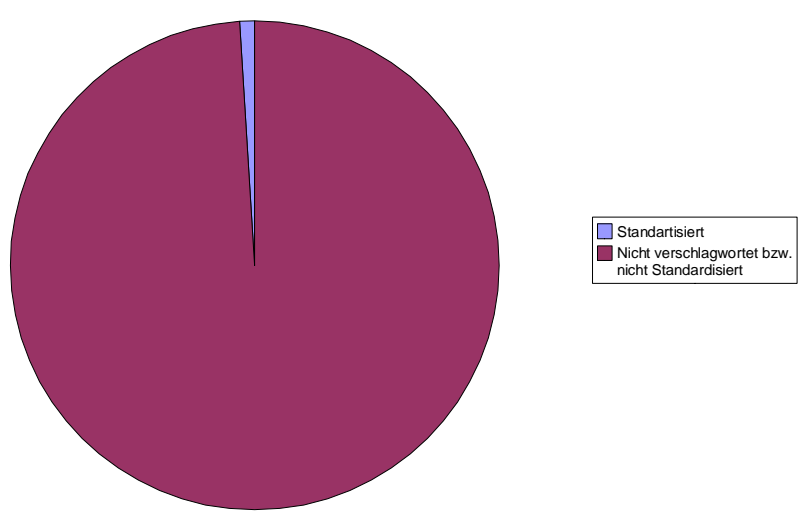

Beim HBZ sind demnach die Daten zweier Titel, 0,96 \% der Gesamtmenge, übernehmbar. 


\subsubsection{7 ÖNB}

\begin{tabular}{|c|c|c|c|}
\hline Titel. Insg. & Vorhanden & Titel mit SW & SW standardisiert \\
\hline 209 & 62 & 62 & 56 \\
\hline
\end{tabular}

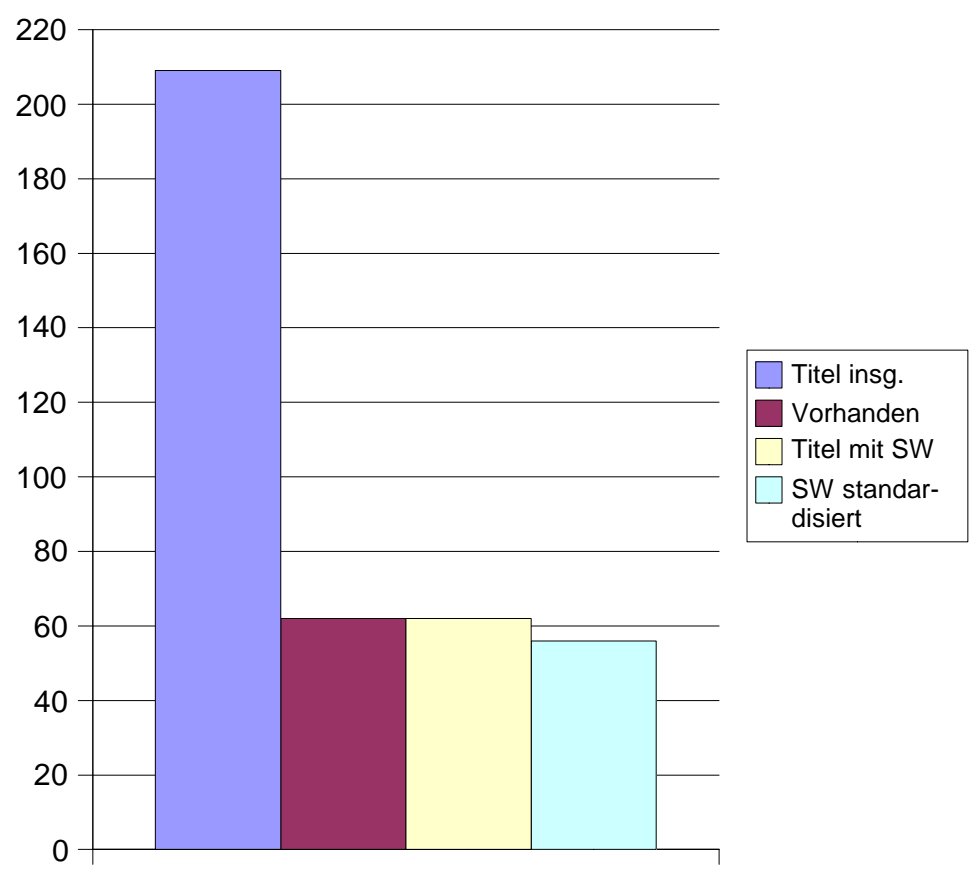

\begin{tabular}{|c|c|c|}
\hline Von 209 Titeln sind & Vorhanden & Nicht vorhanden \\
\hline & $29,67 \%$ & $70,33 \%$ \\
\hline $\begin{array}{c}\text { Von diesen } 29,67 \% \text { sind } \\
\text { per SW }\end{array}$ & Erschlossen & Nicht erschlossen \\
\hline & $100,00 \%$ & $0,00 \%$ \\
\hline
\end{tabular}
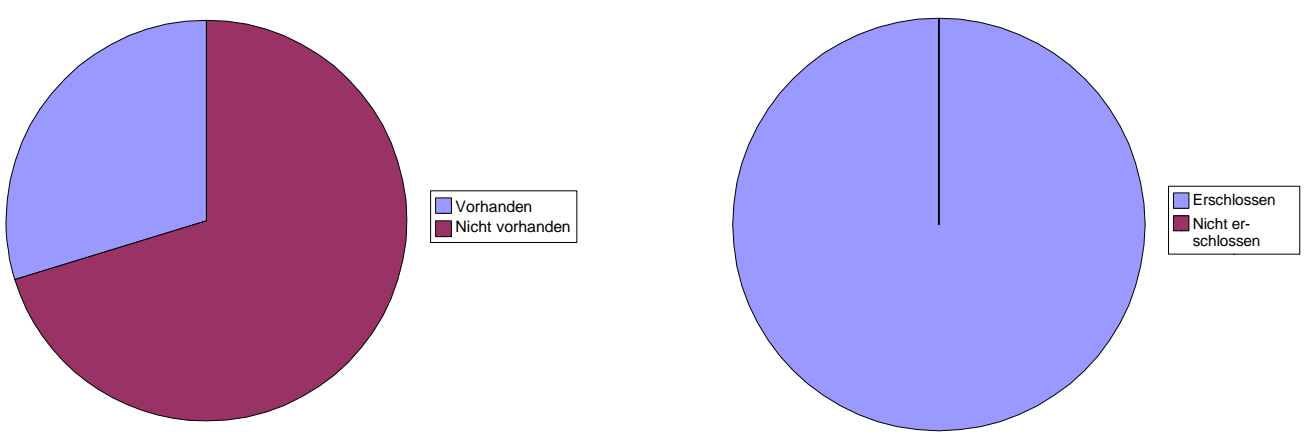


\begin{tabular}{|c|c|c|}
\hline Von diesen $100,00 \%$ sind & Übernehmbar & Nicht standardisiert \\
\hline & $90,32 \%$ & $9,68 \%$ \\
\hline $\begin{array}{c}\text { Insgesamt bedeutet dies, } \\
\text { von 209 Titeln sind }\end{array}$ & Erschlossen & Nicht erschlossen \\
\hline & $29,67 \%$ & $60,33 \%$ \\
\hline
\end{tabular}
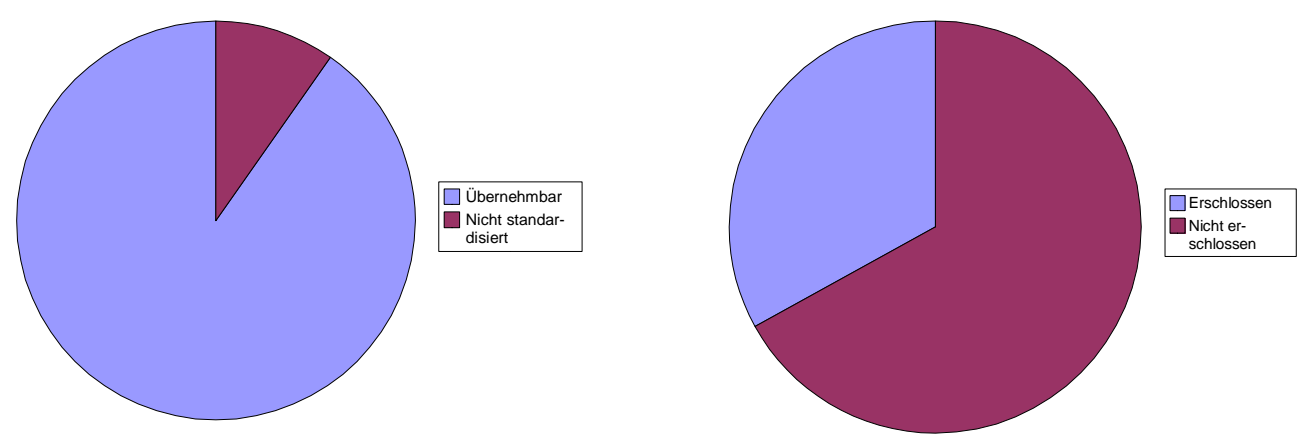

Übernehmbare Erschließungsdaten bezogen auf die Gesamtmenge:

\begin{tabular}{|c|c|}
\hline Standardisiert & $\begin{array}{c}\text { Nicht verschlagwortet, bzw. nicht } \\
\text { standardisiert }\end{array}$ \\
\hline $26,79 \%$ & $73,21 \%$ \\
\hline
\end{tabular}

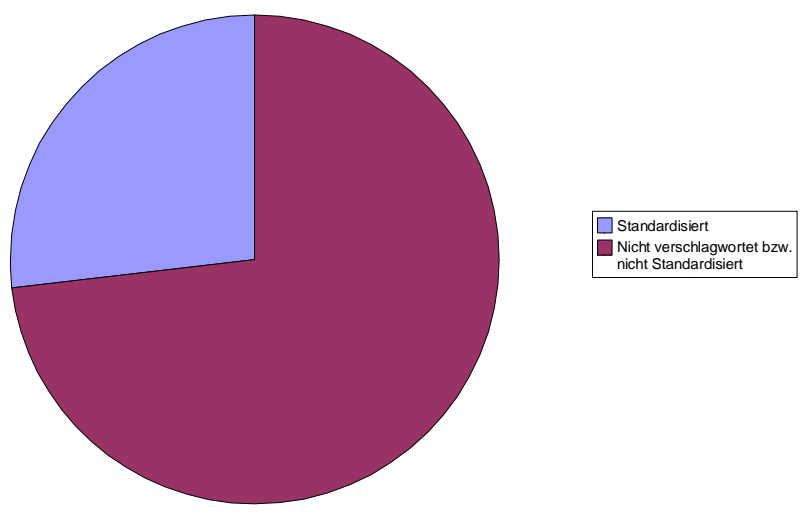

Bei der Österreichischen Nationalbibliothek sind die inhaltlichen Erschließungsdaten von 29,79 \% der überprüften Gesamttitelzahl für andere Verbünde nutzbar. Wären mehr als 62 Titel in der ÖNB vorhanden, würde diese Prozentzahl wohl wesentlich höher ausfallen. Dies wird deutlich, wenn man berücksichtigt, dass bei den in der ÖNB vorhandenen Titeln die Daten von 90,32 \% übernehmbar sind. 
5.3.9.8 HeBis

\begin{tabular}{|c|c|c|c|}
\hline Titel insg. & Vorhanden & Titel mit SW & SW standardisiert \\
\hline 209 & 37 & 5 & 2 \\
\hline
\end{tabular}

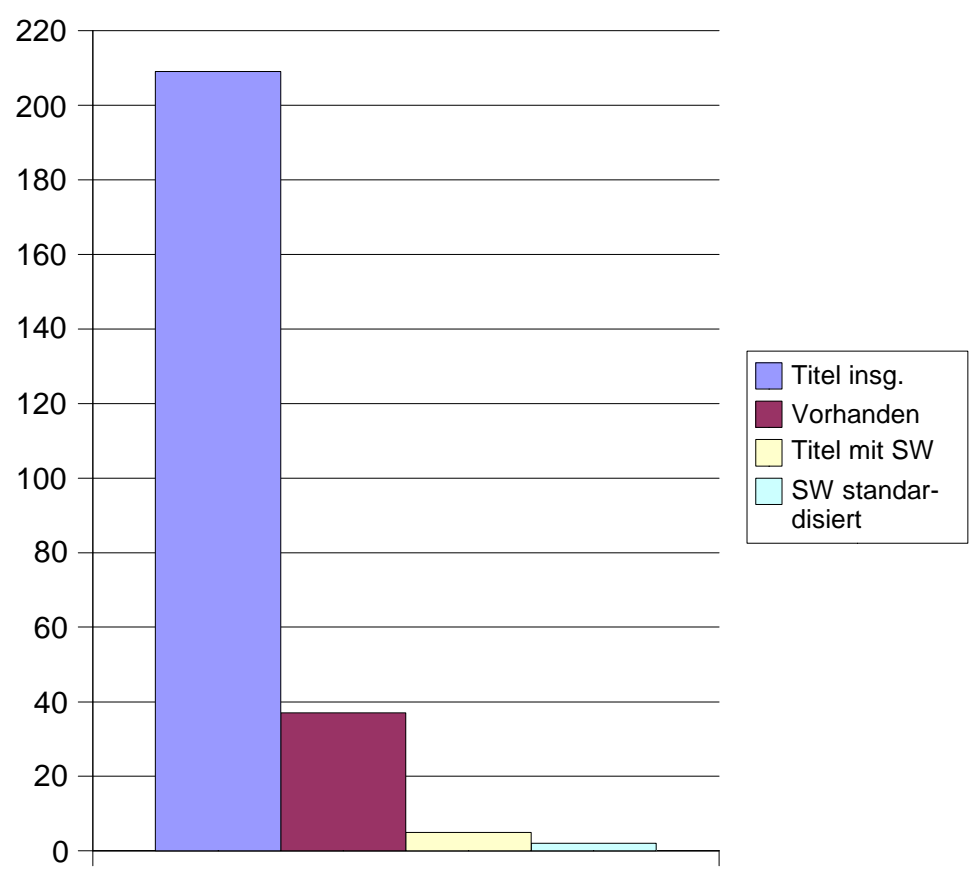

\begin{tabular}{|c|c|c|}
\hline Von 209 Titeln sind & Vorhanden & Nicht vorhanden \\
\hline $\begin{array}{c}\text { Von diesen } 17,70 \% \text { sind } \\
\text { per SW }\end{array}$ & Erschlossen & $82,30 \%$ \\
\hline & $13,51 \%$ & Nicht erschlossen \\
\hline
\end{tabular}
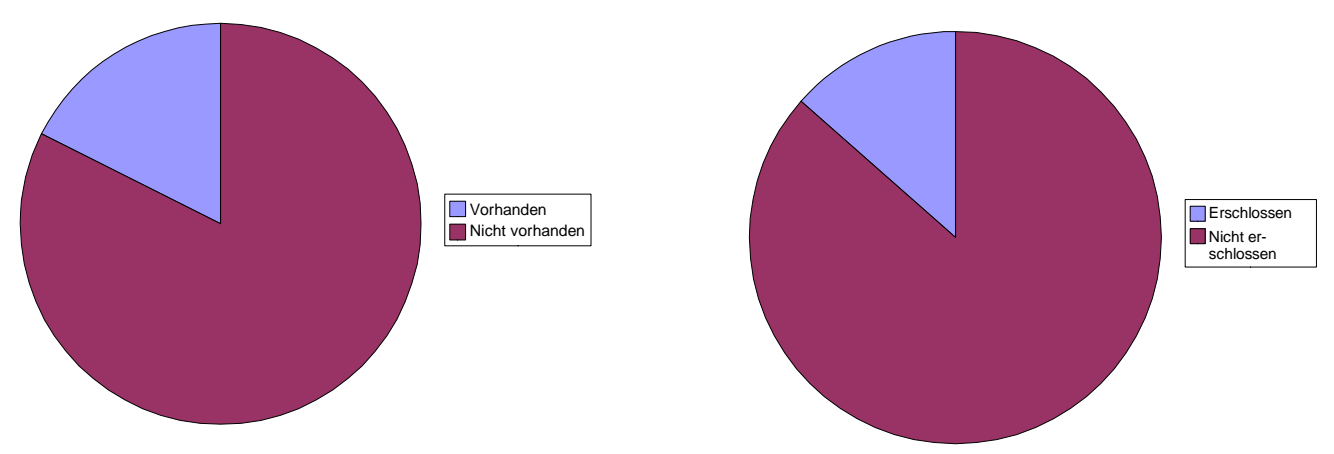


\begin{tabular}{|c|c|c|}
\hline Von diesen 13,51\% sind & Übernehmbar & Nicht standardisiert \\
\hline & $40,00 \%$ & $60,00 \%$ \\
\hline $\begin{array}{c}\text { Insgesamt bedeutet dies, } \\
\text { von 209 Titeln sind }\end{array}$ & Erschlossen & Nicht erschlossen \\
\hline & $2,39 \%$ & $97,61 \%$ \\
\hline
\end{tabular}
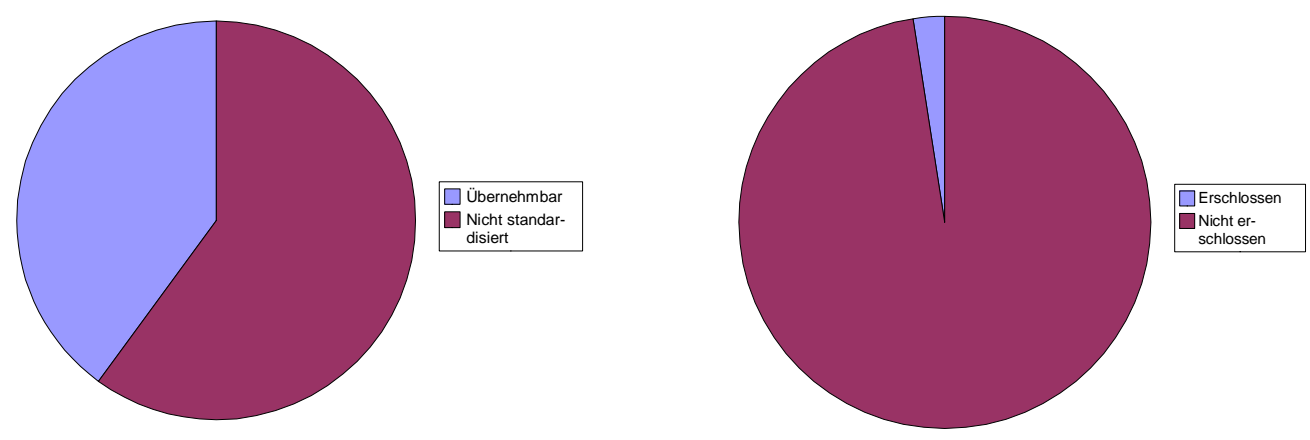

\section{Übernehmbare Erschließungsdaten bezogen auf die Gesamtmenge:}

\begin{tabular}{|c|c|}
\hline Standardisiert & $\begin{array}{c}\text { Nicht verschlagwortet bzw. nicht Standar- } \\
\text { disiert }\end{array}$ \\
\hline $0,96 \%$ & $99,04 \%$ \\
\hline
\end{tabular}

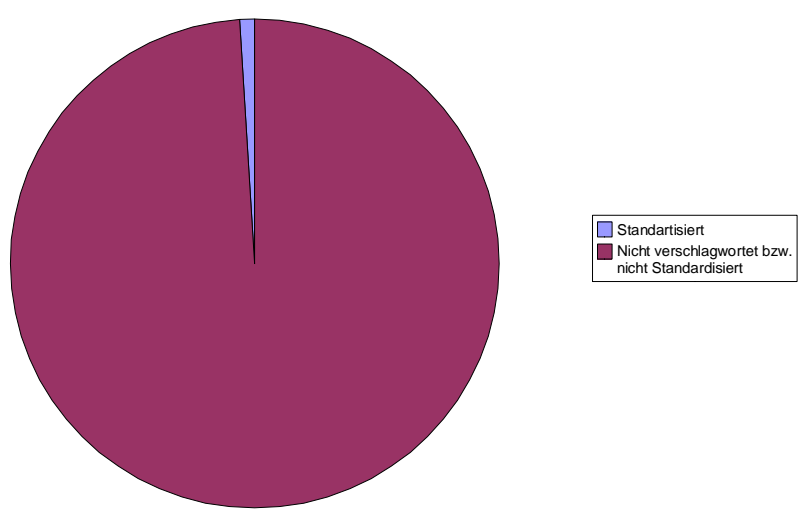

Im HeBis sind ebenso wie im HBZ 0,96 \% übernehmbar. Jedoch liegt dies u.a. auch an der geringen Anzahl von Titeln, die im HeBis vorhanden sind. 


\subsubsection{KOBV}

Von den 20 ermittelten Titeln aus dem KOBV waren keine per Schlagwort erschlossen.

\subsubsection{Fazit}

Anhand dieser Zahlen ist deutlich erkennbar, dass vor allem in Deutschland in den verschiedenen Bibliotheken und Verbünden nur sehr wenige Titel des Altbestandes inhaltlich erschlossen sind.

Eine Ausnahme in dieser Betrachtung stellt die Österreichische Nationalbibliothek dar. Es bestätigt sich in der Untersuchung die in Kap. 4.8. getätigte Auskunft der Bibliothek über ihre Vorgehensweise in der Sacherschließung ihrer Altbestände.

In Deutschland hebt sich der Gemeinsame Bibliotheksverbund von den anderen getesteten Bibliotheken und Verbünden ab. Zählt man die mit Schlagwörtern, Gattungsbegriffen und Basisklassifikation erschlossenen Titel zusammen, ergiebt sich eine relativ hohe Erschließungsdichte, wobei sich einzelne Titel auch hier wiederum überschneiden. Bezieht man die Titel, die sowohl mit einem oder mehreren Gattungsbegriffen als auch mit einer Notation der Basisklassifikation versehen wurden, nur einmal in die Berechnung mit ein, handelt es sich hierbei um 99 Titel, die standardisierte Inhaltserschließungsdaten tragen. Dies hängt v.a. auch mit den aus der SUB Göttingen in den GBV eingespielten Daten zusammen. Durch deren Verantwortung für das Zeitsegment 1701-1800 innerhalb der Arbeitsgemeinschaft Sammlung Deutscher Drucke (vgl. Kap. 3.1. und 4.3.) sind zahlreiche Titel innerhalb des GBV mit Gattungsbegriffen und Notationen auf Grundlage der Basisklassifikation versehen.

In den weiteren getesteten Verbünden fallen die Ergebnisse eher ernüchternd aus. Sowohl im SWB, im HBZ, im HeBis als auch im KOBV gibt es in diesem Bereich noch erheblichen Nachholbedarf. Auch in der Staatsbibliothek Berlin sind relativ wenige Titel mit Hilfe eines Schlagwortes erschlossen. Allerdings muss man dabei bedenken, dass die Staatsbibliothek ihren kompletten Altbestand anhand ihres Alten Realkataloges und der darin verwendeten historischen Systematik erschlossen hat und diese z.T. online recherchierbar ist (vgl. Kap. 4.4.). Zudem bietet eine solche Form der Erschließung die Möglichkeit einer Entwicklung von Konkordanzen (vgl. Kap. 7.3.)

Nach einem Überblick über die verschiedenen möglichen Arten der Inhaltserschließung folgen in Anbetracht der ernüchternden Zahlen dieser Untersuchung einige Überlegungen, wie man eine Erschließung von Historischen Beständen effektiver gestalten könnte. 


\section{$6 \quad$ Verwendete Inhaltserschließung}

Wie gesehen gibt es mehrere Möglichkeiten, Literatur inhaltlich zu erschließen. Nicht alle Methoden haben denselben Arbeitsaufwand und nicht alle Methoden führen in derselben effektiven Weise zu den gewünschten Ergebnissen. Meist besteht ein Zusammenhang zwischen Einsatz und Ertrag. Versucht man, Zeit-, Personal- und Kostenaufwand möglichst gering zu halten, muss auf Methoden zurückgegriffen werden, die das eigentliche Ziel nur bedingt erreichen, nämlich die möglichst optimale Bereitstellung der Bestände für den Nutzer. Jedoch lässt sich andererseits bereits mit relativ geringem Aufwand ein sehr nützliches Ergebnis erzielen, siehe unter anderem die Erschließung mit Gattungsbegriffen beim VD 17.

Die verschiedenen Möglichkeiten, Bibliotheksbestände inhaltlich zu erschließen, werden im Folgenden unter besonderer Berücksichtigung historischer Bestände und anhand von Beispielen aufgezeigt.

\subsection{Verbale Erschließung}

\subsubsection{Stichwörter}

„Stichwörter werden Texten durch direkte Extraktion unverändert entnommen, um diese in aller Kürze zu kennzeichnen. Vorzugsweise entnimmt man sie dem Titel als Titelstichwörter. Sie können aber auch aus dem gesamten Textkorpus als Textstichwörter gewonnen werden. Da sie bei der Extraktion aus ihrem Zusammenhang gerissen werden, sollte man korrekterweise von Stichwörtern und nicht von Stichworten sprechen. " 100

Die Anwendung von relevanten Stichwörtern als inhaltserschließende Maßnahme ist unter allen Maßnahmen diejenige, mit der sich am wenigsten Aufwand verbindet. Bei moderner Literatur kann man Stichwörter zusätzlich maschinell übernehmen, z.B. bei Digitalisaten aus dem Volltext, aus eingescannten Abstracts und aus eingescannten Inhaltsverzeichnissen. Bei alten Drucken stößt man jedoch auf einige Probleme. (siehe Kap. 7.5.)

Bei einem Titel, der den Inhalt eines Werkes treffend und prägnant ausdrückt, ist im Onlinekatalog von vornherein eine Erschließung mit Hilfe von Stichwörtern gegeben. Musste man früher bei einem nach Stichwörtern geordneten Katalog diese Stichwörter auf den Katalogkarten noch gesondert kennzeichnen und nach diesen dann alphabetisch ordnen, ist dies bei der modernen EDV-Technik im OPAC nicht mehr notwendig. Im OPAC dient im Prinzip jedes Wort eines Titels und Untertitels neben der formalen gleichzeitig der inhaltlichen Erschließung, wenn es denn aussagekräftig genug in Bezug auf das behandelte Thema ist und das Kategorienfeld, in dem es sich befindet, der

100 Löffler, Karl; Umstätter, Walther; Wagner-Döbler, Roland (2005), hier S. 52 
Recherche zugänglich ist. Im Prinzip kann jedes Wort einer Titelaufnahme als Stichwort behandelt werden. So kann man eine Recherche im OPAC genauso wie die Suche in Suchmaschinen wie z.B. Google angehen, und zwar nach dem Prinzip der Stichwortsuche. Bei einer Suche nach einem bestimmten Begriff werden Dokumente gefunden, die den Suchbegriff entweder im Titel, im Untertitel oder auch im Volltext beinhalten. Ob man damit allerdings auch wirklich ein für sich relevantes Dokument gefunden hat, ist nicht garantiert. Sucht man ein bestimmtes Werk, z.B. über die Französische Revolution, so kann man einfach die Phrase Französische Revolution entweder in die Titelsuche oder in einer Suche in allen Feldern eingeben und wird dann mit großer Gewissheit auf mehrere Werke stoßen, die diese Phrase im Titel oder Untertitel tragen und auch genau dieses Thema behandeln. Bei alten Drucken stösst man hierbei jedoch oft auf Probleme. Der Hauptgrund hierfür liegt in der unterschiedlichen Orthographie von Begriffen, etwa im Vergleich des 17. Jahrhunderts zur heutigen Zeit. So z.B. bei folgendem Titel:

Lange, Johann F.:

Beschreibung der königlichen Residenzstadt Coppenhagen und der königlichen Landschlösser. 1786

Wenn ein Bibliotheksbenutzer ein historisches Werk über Kopenhagen sucht, kann dieses Werk weder bei der Suche über die formale Beschreibung gefunden werden, noch ist eine maschinelle Indexierung möglich. Dass die betreffende Person mit der nicht mehr geläufigen Schreibweise Coppenhagen recherchiert, ist sehr unwahrscheinlich. Um es trotzdem mit wenig Aufwand für den Nutzer auffindbar zu machen, kann manuell das Stichwort Kopenhagen angelegt werden.

In solchen Fällen ist es also nicht möglich, den Titel durch seine Formalerschließungsdaten aufzufinden. Um es problemlos suchbar zu machen, muss eine grammatikalisch und orthographisch an die moderne Schriftsprache angepasste, evtl. normierte Schreibweise in ein entsprechendes, dafür reserviertes Feld eingefügt werden. Ebenso verfahren muss man natürlich auch bei Stichwörtern aus anderen Sprachen, die man ins Deutsche übersetzt.

Der Nachteil bei der Vergabe von Stichwörtern ist natürlich die nur sehr oberflächliche Erschließung. Dazu besteht die bei nicht-normierten Stich- oder Schlagwörtern immer existierende Gefahr, dass der Nutzer mit anderen Begriffen als den bei der ErschlieBung vergebenen sucht, da dieser in jenem Fall ja nicht auf einen Index zurückgreifen kann. Um diese Gefahr zu verringern, kann man für das festgelegte Stichwort auch maschinell Synonyme generieren lassen.

Die Vergabe von Stichwörtern überschneidet sich insofern mit der Vergabe von freien Schlagwörtern, da diese ebenfalls nicht normiert sind. 


\subsubsection{Freie und gebundene Schlagwörter}

„Schlagwörter werden im Gegensatz zu den Stichwörtern nicht unverändert den Texten entnommen, sondern vereinheitlicht. Wir unterscheiden zwischen dem ungebundenen oder freien und dem gebundenen Schlagwort."

„Das ungebundene oder freie Schlagwort kann über mehr oder minder klar vorgegebene Regeln jederzeit neu geschaffen werden, so wie es sich aus den Dokumenten ergiebt. Es unterliegt keiner Kontrolle in einer Schlagwortliste. "101

Im Gegensatz zu einem Stichwort wird ein Schlagwort definitiv manuell bzw. maschinell angelegt. Da es sich nicht wie das Stichwort bereits im Titel befindet, kann es auch nicht durch eine einfache Recherche nach Formaldaten gefunden werden. ${ }^{102}$

Die Vorteile eines freien Schlagwortes sind in etwa dieselben wie bei der Vergabe von Stichwörtern. Es ist schnell angelegt und man muss sich nicht an bestimmte Listen halten. Man kann dasjenige Wort als freies Schlagwort bestimmen, welches man für besonders geeignet hält. Zudem hat man bei freien Schlagwörtern eventuell die Möglichkeit, sich besser der Terminologie der alten Drucke anzupassen. Als freies Schlagwort wird ebenfalls das Wort in der Grundform benutzt. Wenn man ein solches ansetzt, muss dies jedoch im bibliographischen Teil des Datensatzes in ein anderes Feld gesetzt werden als die gebundenen Schlagwörter, evtl. in ein eigenes Feld für freie Schlagwörter.

Es folgt ein Beispiel für die Ansetzung von freien Schlagwörtern. Anschließend wird das selbe Beispiel in den jeweiligen Kapiteln noch zweimal betrachtet, zuerst mit der Ansetzung nach der SWD und danach mit der Ansetzung nach den RSWK.

Leitfaden zur Nordischen Althertumskunde

hrsg. Von der königl. Ges. für Nordische Altherthumskunde. 1837.

Als freie Schlagwörter kann man hier ansetzen:

Leitfaden und Nordische Staaten und Altertumskunde

Die Anwendung freier, nicht normierter Schlagwörter bei Altbeständen bietet für denjenigen, der die Erschließung durchführt, die Chance, zeitsparend und unabhängig eine verbale Suchmöglichkeit zu schaffen.

101 Löffler, Karl; Umstätter, Walther; Wagner-Döbler, Roland (2005), hier S. 52

102 Natürlich ist es häufig der Fall, dass Schlag- und Stichwort übereinstimmen. Somit kann das Schlagwort natürlich auch bereits im Titel vorkommen. 
„Das gebundene Schlagwort unterliegt einem kontrolliertem Vokabular, das in Form eines Thesaurus, aber auch in Form einer einfachen Schlagwortliste vorliegen kann. Ihnen sind die Schlagwörter zu entnehmen. "103

Ein gebundenes Schlagwort, auch als Deskriptor bezeichnet, unterliegt dagegen der Kontrolle eines Thesaurus, d.h. eines kontrollierten Vokabulars. Es dürfen zur Inhaltsbeschreibung nur Begriffe aus diesem Vokabular verwendet werden. Dabei gilt meist das „Prinzip des engen Schlagworts“, d.h. der Inhalt des Dokuments muss mit Hilfe der Deskriptoren so genau wie möglich beschrieben werden um eine möglichst tiefe Erschließung zu garantieren.

Als Schlagwortliste bei der Altbestandserschließung könnte man wie für die moderne Literatur die SWD heranziehen, wobei jedoch ein Problem bei der Anwendung von Schlagwörtern aus modernen Thesauri bei historischen Drucken besteht, wie bereits mehrfach gehört. Exemplarisch hierfür ist der Bedeutungswandel des Begriffes „Polizei“ seit der Frühen Neuzeit, den Arnold 1997 anschaulich erläutert. Er bezieht sich dabei zwar auf Klassifikationen, jedoch gilt dies ebenso für die Verschlagwortung:

„Konkretisieren lässt sich das Problem an historischen Leitbegriffen wie beispielweise Aufklärung, Bildung, Bürgertum, Freiheit, Gesellschaft oder Polizei, Politik, Recht usw. Diese Begriffe werden in der Literatur über einen langen Zeitraum mit erheblicher Bedeutungsdifferenz behandelt, die eine Erschließung in unterschiedliche Klassen erfordert. [...]

Das wird vielleicht deutlicher, wenn man den Bedeutungswandel eines dieser Leitbegriffe exemplarisch betrachtet. Zu diesem Zweck sei der Begriff Polizei gewählt. [...] Der Begriff selbst lässt sich kaum exakt definieren. Unter Polizei verstanden die Quellen der Frühen Neuzeit das gesamte Gemeinwesen, Staat, Stadt, ihre Verfassung und die Ständeordnung; in vielen Gesetzen und Erlassungen wird die Wohlfahrt des Landes auf die "gute Polizei“ begründet. Erst in der zweiten Hälfte des 18. Jahrhunderts wird Polizei im Zusammenhang mit dem Ausbau der Behörden als Inbegriff der Staatsordnung, was Friedensicherung und Rechtswahrung bedeutet, allmählich von der Außenpolitik, der Finanzverwaltung und dem Militärwesen ab- und auf die Aufgaben eingegrenzt, die heute mit dieser Institution verbunden werden. Eine weitere Bedeutungsnuance ist zu bedenken. Die Texte des 15. bis 18. Jahrhunderts bezeichnen mit Polizei zuweilen auch die Gesetze, die zur Herstellung und Erhaltung guter Ordnung dienten und die Kleiderordnungen, das Verhalten bei Festlichkeiten, die Berufsausübung, Handelsverträge sowie Rechtsbeziehungen aller Art regelten." 04

Anhand diesem Beispiels wird klar, dass man bei der Anwendung moderner Termini auf historische Werke auf erhebliche Probleme stossen kann.

Eine mögliche Lösung für dieses Problem könnte jedoch eventuell darin bestehen, dass man durch die Konversion vorhandener alter Schlagwortkataloge zahlreiche sinnvolle ältere Begriffe in die Datenbanken der Verbundkataloge einspielt, auch wenn die-

${ }^{103}$ Löffler, Karl; Umstätter, Walther; Wagner-Döbler, Roland (2005), hier ebenfalls S. 52

104 Vgl. Arnold, Werner (1997), hier S. 337. 
se nicht normiert sind. ${ }^{105}$ Man könnte diese Begriffe sowohl in einem eigenen, als auch in einem gemeinsamen Index ablegen, zusammen mit SWD-Schlagwörtern und weiteren Stichwörtern. Mit der Verschlagwortung weiterer alter Literatur kämen mit der Zeit zahlreiche Begriffe hinzu, so dass die Menge an alten Bezeichnungen zunimmt und diese Indices eventuell sinnvoll strukturiert werden könnten. Diese könnten dann dem Nutzer zur gezielten Recherche angeboten werden. So schreibt Arnold: „Die Recherchemöglichkeit mit nicht normiertem Vokabular muß für diese Texte erweitert werden, vor allem durch Fachbegriffe aus den alten Drucken selbst, beispielweise durch eine Auswertung der Inhaltsverzeichnisse. Die Historisierung der Terminologie in den Klassifikationen, in den Registern und Normsätzen, wie diese Notwendigkeit mangels einer besseren Bezeichnung genannt werden soll, würde unsere Hilfsmittel zwar komplizierter, aber auch effizienter für ihre Funktion als Forschungsinstrumente werden lassen." "106

Nun nochmal dasselbe Beispiel wie bei den ungebundenen Schlagwörtern, diesmal jedoch normiert nach der SWD:

\section{Leitfaden zur Nordischen Althertumskunde}

hrsg. Von der königl. Ges. für Nordische Altherthumskunde. 1837.

Als normierte Schlagwörter kann man hier ansetzen, so z.B. nach der SWD:

Einführung und Nordische Staaten und Altertumswissenschaft

Man sieht, dass sich Begriffe wie Einführung und Altertumswissenschaft von der Form im Titel unterscheiden. Um die Ansetzungsformen zu ermitteln ist Recherche nötig, der Aufwand der Sacherschließung ist also grösser.

Zudem muss man entscheiden, ob man die Schlagwörter eng oder weit ansetzen will. Eine Art von sehr weiten Schlagwörtern stellen die Gattungsbegriffe dar.

${ }^{105}$ So z.B. den Alten Schlagwortkatalog der SUB Hamburg, den Schlagwortkatalog der HAAB Weimar oder den Schrettinger-Katalog der Bayerischen Staatsbibliothek. (Vgl. dazu Kap. 4.) Das Schlagwortprinzip existiert seit 1820, als Martin Schrettinger den Systematischen Katalog der Münchener Hofbibliothek als den später nach ihm benannten Schlagwortkatalog weiterführte, um den gewaltigen Zuwachs an Literatur während der Säkularisation bewältigen zu können. Dadurch verlief die Katalogisierung der Bücher unabhängig von der Aufstellung. Vgl. u.a. Löffler, Karl; Umstätter, Walther; Wagner-Döbler, Roland (2005), hier S. 120. Vgl. zu dieser Thematik auch Rückert, Ingrid (2003)

${ }_{106}$ Arnold, Werner (1997), hier S. 337 


\subsubsection{Gattungsbegriffe}

Die Vergabe von Gattungsbegriffen wurde bereits in Kapitel 3.2.2. ausgiebig vorgestellt. Für eine grobe inhaltliche Erschließung Gattungsbegriffe zu verwenden hat zahlreiche Vorteile, aber auch einige Nachteile. Gattungsbegriffe, bzw. Fachgruppenbezeichnungen sind relativ schnell vergeben. Man kann große Mengen an Literatur vergleichsweise schnell erschließen, der Aufwand bleibt dabei gering. Jedoch hat man trotz des geringen Aufwands einen großen Nutzen. Man kann wie in Kap. 3.2.2. bereits erwähnt, große Datenmengen einschränken und durchaus gezielt recherchieren. Bei der Untersuchung anhand der Systematikgruppe IV.18 (Geschichte der skandinavischen Reiche) waren von den 209 Titeln 27 im VD 17 verzeichnet, 23 davon waren durch ein oder mehrere Gattungsbegriffe erschlossen. Dies zeigt, dass die Anwendung von Gattungsbegriffen durchaus sinnvoll ist, da man so $85,19 \%$ der vorhandenen Drucke inhaltlich auffinden kann, auch wenn dies nur auf einer groben Ebene möglich ist.

Gattungsbegriffe sind somit für die Altbestandserschließung sehr nützlich, daher werden sie bestimmt auch bei zukünftigen Erschließungsprojekten häufig verwendet werden, sei es bei kompletten Beständen oder nur in speziellen Bereichen. Vor allem, wenn die finanziellen und personellen Ressourcen knapp sind, aber dennoch eine Art inhaltlicher Erschließung erwünscht wird. Dies könnte auch für ein eventuelles VD 18 gelten, dort gegebenenfalls noch zusammen mit einer Klassifikation, z.B. der Basisklassifikation, wie bereits praktiziert in der Sammlung Deutscher Drucke 1701-1800.

\subsubsection{RSWK}

Die Erschließung mit Schlagwortketten wie bei den RSWK ist wohl die zeitaufwendigste Methode, aber auch sehr effizient. Ist es im deutschsprachigen Gebiet bei moderner Literatur mittlerweile selbstverständlich, per RSWK zu erschließen, stösst man bei Altbeständen auf Probleme. Syré stellte die Probleme dar, wie bereits in Kap. 3.1. zitiert, und spricht sich deswegen gegen eine Sacherschließung in solcher Form aus. Auch wurde diese Problematik schon bei den gebundenen Schlagwörtern in Kap. 6.1.2. näher erläutert. Somit ist es fraglich, ob eine Beschlagwortung nach einem Regelwerk wie den RSWK auch für Altbestände allgemein angestrebt werden sollte. Dies dürfte vom Aufwand wohl nur sehr schwer zu leisten sein. Dass es aber möglich ist, zeigt das Beispiel der Österreichischen Nationalbibliothek, die ihren Altbestand ja durchgehend mit RSWK erschließt (vgl. Kap. 4.8.). Auch ist bei der Testrecherche in Kap. 5.2. festgestellt worden, dass im Dreiländerkatalog mehr Titel mit den RSWK verschlagwortet als mit der RVK klassifiziert sind. Es sollte überlegt werden, ob man nicht Möglichkeiten entwickeln könnte, die RSWK für eine zukünftige Altbestandserschließung anzupassen. 
Nun folgt noch einmal dasselbe Beispiel. Dieses Mal soll es darstellen, wie die Ansetzung der Schlagwörter nach den RSWK aussehen müsste.

\author{
Leitfaden zur Nordischen Althertumskunde \\ hrsg. Von der königl. Ges. für Nordische Altherthumskunde. 1837.
}

Nach den RSWK muss man folgendermaßen erschließen:

Nordische Staaten ; Altertumswissenschaft ; Einführung

Die Relationen zwischen den Begriffen werden also ausgedrückt, da die Reihenfolge der Schlagwörter innerhalb der Schlagwortkette im Regelfall den verschiedenen, festgelegten Kategorien folgt, wobei auch mehrere Schlagwörter einer Kategorie in einer Kette verwendet werden können (Personenschlagwort, geographisches Schlagwort, Sachschlagwort, Zeitschlagwort und Formschlagwort). Jeder behandelte Gegenstand erhält dabei eine Kette ${ }^{107}$ und wird durch diese Schlagwortkette repräsentiert. ${ }^{108}$ Schlagwortketten bilden somit Kurz-Abstracts des Dokumenteninhalts. ${ }^{109}$ Im obigen Beispiel bildet Nordische Staaten das geographische Schlagwort, Altertumswissenschaft das Sachschlagwort und Einführung das Formschlagwort. Es handelt sich also um eine Einführung in die Altertumswissenschaften der Nordischen Staaten. Dies ist ein großer Vorteil für den Benutzer, der Aufwand für den Erschließer ist jedoch wesentlich höher.

\title{
6.2 Klassifikatorische Erschließung
}

„Die Klassifikatorische Sacherschließung beruht auf Klassifikationssystemen mit (meist) hierarchisch geordneten Haupt- und Untergruppen oder Klassen, die durch Notationen bezeichnet werden. Bei der Sacherschließung durch Klassifizieren wird jede Publikation einer Klasse oder Systemstelle zugeordnet. Alle Bücher über das gleiche Thema erhalten die gleiche Notation und sind über diese zu ermitteln." "10

Wie man anhand den bisherigen Ausführungen sehen konnte, sind in den einzelnen Bibliotheken die Altbestände gewöhnlich mit einer historischen Aufstellungssystematik erschlossen. Diese ist dabei meist von Bibliothek zu Bibliothek unterschiedlich, obwohl es durchaus einzelne Überschneidungen gibt.

\footnotetext{
107 Vgl. RSWK (1998), hier § 13

${ }^{108}$ Vgl. RSWK (1998), hier § 7

${ }^{109}$ Vgl. RSWK (1998), hier § 13

110 Hacker, Rupert (2000), hier S. 201
} 
Historische Systeme sind z.B. das Hartwigsche System, die Methode Eppelsheimer bzw. der Mainzer Sachkatalog, ${ }^{111}$ das Schleiermachersche System, ${ }^{112}$ das Gülichsche Katalogsystem ${ }^{113}$ oder das System des Berliner ARK, welches in seinen Grundlagen auf das Schleiermachersche System zurückgeht. ${ }^{114}$ Bei den Untersuchungen zeigte sich, dass in den getesteten Bibliotheken die Literatur auch durchgehend anhand dieser alten Systematiken erschlossen war. Diese Gewissheit sollte als Basis dienen, Möglichkeiten zu finden, um diese Systematiken auch überregional und nicht nur im lokalen Katalog nutzbar zu machen.

Bei modernen Klassifikationen werden zur Klassifizierung alter Bestände hauptsächlich die Regensburger Verbundklassifikation (u.a. UB Eichstätt, vgl. Kap. 4.1.) und die Basisklassifikation (u.a. SUB Göttingen, vgl. Kap. 4.3.) herangenommen. Die Dewey Decimal Classification (DDC) und weitere Klassifikationen spielen bei bisherigen ErschlieBungsvorgängen noch keine Rolle. Der Vorteil der RVK liegt eindeutig an ihrer weiten Verbreitung, die eine bessere Nutzung von Fremddaten gewährleistet, an ihrer Anwendung im Dreiländerkatalog und an ihrer bequemen Recherchierbarkeit inklusive Register, Begriffs- und Notationssuche unter RVK-Online. ${ }^{115}$ Zudem wird sie laufend aktualisiert, ist fein untergliedert und die Registereinträge sind an die SWD angepasst. Der Aufbau der Basisklassifikation ${ }^{116}$ ist eher grob. Sie „ist eine monohierarchische Klassifikation, die für die Recherche in Opacs wissenschaftlicher Bibliotheken in Kombination mit der Recherche nach Schlagwörtern entwickelt wurde. Insofern hat die BK keine eigenständige Erschließungsfunktion, sondern soll Erschließung vermittels Schlagwörtern optimieren, indem die Suche anhand von Schlagwörtern zugleich auf mehr oder minder umfangreiche Wissenschaftsgebiete eingegrenzt werden kann."117 Ihre Anwendung ist durch ihre Benutzung bei der Sammlung Deutscher Drucke des 18. Jahrhunderts bereits für Altbestände erprobt. Dort wird sie zusammen mit Gattungsbegriffen

111 Vgl. dazu Riplinger, Thomas (2004): Die Bedeutung der Methode Eppelsheimer für Theorie und Praxis der bibliothekarischen und der dokumentarischen Sacherschließung. In: Bibliothek. Forschung und Praxis, Jg. 28, H. 2, S. 252-262. Online verfügbar unter http://www.bibliothek-saur.de/2004_2/252-262.pdf, zuletzt geprüft am 17.02.2008.

112 Nähere Erläuterungen zum Schleiermacherschen System gibt es u.a. bei Löffler, Karl; Umstätter, Walther; Wagner-Döbler, Roland (2005), hier S. 124

${ }^{113}$ Nähere Erläuterungen hierzu gibt es ebenfalls bei Löffler, Karl; Umstätter, Walther; WagnerDöbler, Roland (2005), hier S. 102

${ }^{114}$ Krabbe, Wilhelm; Luther, Wilhelm Martin (1953): Lehrbuch der Bibliotheksverwaltung. Stuttgart: Hiersemann. Online verfügbar unter http://www.ib.hu-berlin.de/ wumsta/kralu/kul4.html, zuletzt geprüft am 17.02.2008, hier S. 61

${ }^{115}$ RVK-Online (2008). Online verfügbar unter http://www.bibliothek.uniregensburg.de/rvko_neu/, zuletzt geprüft am 17.02.2008.

${ }^{116}$ Einzusehen ist die Basisklassifikation unter Basisklassifikation (2004). 3., erweiterte Ausgabe 2000. Überarbeitet durch die Facharbeitsgruppe Sacherschließung. Letzte Aktualisierung: 1. Ergänzungslieferung Dezember 2004. Gemeinsamer Bibliotheksverbund (GBV). Online verfügbar unter http://www.gbv.de/vgm/info/mitglieder/02Verbund/01Erschliessung/ 05Sacherschliessung/Sacherschliessung_0448.pdf, zuletzt geprüft am 17.02.2008.

117 Umlauf, Konrad (2006): Einführung in die bibliothekarische Klassifikationstheorie und -praxis. Mit Übungen. Institut für Bibliotheks- und Informationswissenschaft der Humboldt-Universität zu Berlin. (Berliner Handreichungen zur Bibliotheks- und Informationswissenschaft, 67). Online verfügbar unter http://www.ib.hu-berlin.de/\%7Ekumlau/handreichungen/h67/\#rvk, zuletzt aktualisiert am 20.12.2006, zuletzt geprüft am 17.02.2008. 
vergeben. Eventuell wird diese Kombination auch bei einem zukünftigen VD 18 benutzt. Zudem wird die Basisklassifikation seit 2007 auch von der Österreichischen Nationalbibliothek für ihren Altbestand verwendet, wie in Kap. 4.8 erfahren. Diese Vergabe soll zukünftig sogar noch ausgebaut werden. Die Dewey Decimal Classification wurde im Bereich der Altbestandserschließung noch nicht erprobt. Für sie spricht die Entwicklung einer Konkordanz zur SWD und ihre geplante Konkordanz zur RVK (s. Kap. 7.3.) sowie die Entwicklung der DDC-Deutsch. ${ }^{118}$

Dabei ist die Anwendung moderner Klassifikationen auf historische Bestände ebenso problematisch wie die Anwendung moderner Begriffe als Schlagwörter. Arnold erläutert dieses Problem 1997 ausführlich:

„Die inhaltlichen Herausforderungen lassen sich folgendermaßen skizzieren. Texte der frühen Neuzeit bedürfen in hohem Maße der terminologischen Identifizierung und Interpretation, da die Quellen selbst - das betrifft vor allem naturwissenschaftliche Werke - ihr Vokabular in mehrfachem Sinn verwenden oder keine Definition benutzen, sondern aufgrund der fehlenden Fachterminologie die Phänomene nur beschreiben, aber nicht wirklich zuordnen. [...]

Für die klassifikatorische Erschließung ist daher zu bedenken, daß die Begriffe, die für die Konstituierung eines Sachzusammenhangs benötigt werden und die in ein Schlagwortregister Aufnahme finden sollen, vermutlich einen höheren Differenzierungsgrad als moderne Literatur erfordern, durch den einerseits über Verweisungen ein Kontext zu Begriffen weiterer Klassen und andererseits das sich wandelnde Begriffsverständnis im zeitlichen Verlauf erläutert werden soll. [...]

Die Klassifikation älterer Drucke benötigt [...] eine erhebliche Toleranz hinsichtlich der Möglichkeit, vom Kanon der gängigen Hilfsmittel abzuweichen, der für die Normierung von Schlagwörtern benutzt werden soll. "“19

118 Dewey-Dezimalklassifikation (DDC) (2008). Unter Mitarbeit von Heidrun Alex. Deutsche Nationalbibliothek. Bei entsprechender Lizenz online verfügbar unter http://www.ddc-deutsch.de/, zuletzt aktualisiert am 25.01.2008, zuletzt geprüft am 17.02.2008.

${ }^{119}$ Arnold, Werner (1997), hier S. 336-337 


\section{Entwicklungsmöglichkeiten}

Beim Modellieren einer wünschenswerten Situation muss man die Ursachen berücksichtigen, die zur Begründung der mangelnden Erschließungssituation angeführt werden. Es ist nicht abzusehen, dass sich die Situation für Bibliotheken hinsichtlich ihrer Etats und damit verbunden die Zahl ihrer qualifizierten Mitarbeiter verbessern wird, damit man grundlegend inhaltlich erschließen könnte. Es wird wohl auch in Zukunft keine umfassende Sacherschließung von Altbeständen geben, eine komplett durchgeführte Inhaltserschließung ist gar utopisch. Jedoch ist es durchaus möglich und vor allem wünschenswert, diese zu verbessern.

Bibliotheken sollten dafür sorgen, dass die Qualität der bliothekarischen Erschließung mit der durch zahlreiche Digitalisierungen zunehmenden weltweiten Erreichbarkeit von Alten Drucken im Internet Schritt hält. Als weitere Entwicklung schreitet die Retrokatalogisierung von alten Bibliothekskatalogen voran, wie bereits mehrfach in dieser Arbeit erwähnt. Dies schließt folglich ein, dass die Zahl der formal nachgewiesenen Werke stark ansteigt. Mit diesem Anstieg der formalen Nachweise steigt auch der Wunsch nach inhaltlichen Zugriffsmöglichkeiten.

Eine große Chance bietet hierbei ein VD 18, sollte es, wovon auszugehen ist, realisiert werden. Dabei bleibt abzuwarten, wie innerhalb des VD 18 die inhaltliche Erschließung gehandhabt wird. Wünschenswert wäre, wenn sowohl mit Deskriptoren (aller Voraussicht nach Gattungsbegriffen) und einer Klassifikation gearbeitet werden würde. So z.B. mit der Basisklassifikation, die ja schon bei der Sammlung Deutscher Drucke des 18. Jahrhunderts angewandt wird. Die Realisierung eines VD 18 wäre, zusammen mit dem VD 17, ein grosser Schritt in eine bessere inhaltliche Recherchierbarkeit von Historischen Beständen. Aufgrund ihrer weiten Verbreitung sollten bei diesen Überlegungen aber auch weder die RVK noch die RSWK übergangen werden. Fraglich ist dabei allerdings ihre Tauglichkeit für die alten Begrifflichkeiten.

Da eine umfassende Neuerschließung von Altbeständen wohl nicht möglich ist, sollte man versuchen, auf Vorhandenes zurückzugreifen und dieses in Verbindung mit Neuem anzuwenden. 


\subsection{Schalenmodell}

Als Basis für weitere Betrachtungen sowie als allgemeine Grundlage für eine zukünftige kooperative Sacherschließung der historischen Bestände in Deutschland könnte das von Jürgen Krause entwickelte „Schalenmodell“ dienen. ${ }^{120}$ Erstmalig entwickelt wurde es 1996 für die Daten des InformationsZentrum Sozialwissenschaften. ${ }^{121}$ Wurde es ursprünglich für virtuelle Onlinebestände konstruiert, ist es durchaus denkbar, dass man nach solch einem Prinzip auch in der bibliothekarischen Sacherschließung materieller Bestände und des heutzutage damit verbundenen Nachweises in Verbundkatalogen und Metakatalogen wie dem Dreiländerkatalog vorgehen könnte.

Als Grundgedanke muss davon ausgegangen werden, dass mit den ständig wachsenden Verbund- und Metakatalogen und sonstigen kooperativ aufgebauten und genutzten Datenbanken immer größere heterogene Datenwelten entstehen und eine einheitliche Konsistenz zwischen den verschiedenen Daten immer weniger erreichbar sein wird. Das bedeutet, dass die inhaltserschließenden Daten, die jeweils von den einzelnen Bibliotheken und Verbünden in die Datenbanken und Kataloge übermittelt werden, bzw. bereits eingespielt wurden, eine unterschiedliche Erschließungstiefe beinhalten. Die jeweilige Sacherschließungspraxis wird in verschiedenen Bibliotheken unterschiedlich gehandhabt. Dies betrifft nicht nur das verwendete Vokabular und die benutzten Klassifikationen, sondern auch die zu erschließenden Materialien, die Erschließungstiefe und der damit verbundene Aufwand. Aufgrund dessen existieren sowohl Katalogaufnahmen ohne jegliche bibliothekarische Sacherschließung als auch Aufnahmen mit durchaus qualitätsvollen inhaltserschließenden Daten. Dazwischen gibt es verschiedene Klassifikationen, syntaktische Indexate (d.h. die syntaktischen Beziehungen zwischen den Deskriptoren werden in den Indexaten dargestellt; z.B. Schlagwortketten), kontrollierte Deskriptoren (z.B. die Schlagwörter der SWD; Gattungsbegriffe), frei vergebene Schlagwörter, Stichwörter usw. Absprachen zur Normierung und Vereinheitlichung der Daten wären zwar durchaus wünschenswert, jedoch lässt sich dies nur sehr schwer umsetzen, da es mit einem erheblichen Aufwand verbunden ist. So bemerkte Krause bereits 1998: „Normierungsbemühungen und Initiativen zur Akzeptanz und Verbreitung von Metadaten sind eine Voraussetzung für übergreifende Suchprozesse in einer täglich dezentraler werdenden Informationswelt. Sie versuchen, die verloren gegangene Datenhomogenität durch freiwillige Absprachen aller am Informationsprozeß Beteiligter partiell wiederherzustellen. Dies kann jedoch nur teilweise gelingen. Ganz gleich, wie erfolgreich die Einführung von Metadaten in einem Fachgebiet sein wird, die verbleibende Heterogenität der verschiedenen Arten der Inhaltserschließung (automatisch, verschiedene Thesauri, verschiedene Klassifikationen, Unterschiede der erfaßten

120 Vgl. u.a. Krause, Jürgen; Schwänzl, Roland (1998): Inhaltserschließung: Formale Beschreibung, Identifikation und Retrieval, MetaDaten, Vernetzung. Online verfügbar unter http://www.mathematik.uni-osnabrueck.de/projects/slot3/workshop98II/KS.html, zuletzt aktualisiert am 30.04.1998, zuletzt geprüft am 18.02.2008.

${ }^{121}$ Krause, Jürgen (1996): Informationserschließung und -bereitstellung zwischen Deregulation, Kommerzialisierung und weltweiter Vernetzung - Schalenmodel -. InformationsZentrum Sozialwissenschaften. (IZ-Arbeitsbericht, Nr. 6). Online verfügbar unter http://www.gesis.org/ Publikationen/Berichte/IZ_Arbeitsberichte/pdf/ab6.pdf, zuletzt geprüft am 18.02.2008. 
Kategorien) wird zu groß sein, um sie zu vernachlässigen.“ Aus dieser Erkenntnis heraus muss man prüfen, wie man das Problem der Heterogenität, das wohl auch zukünftig existieren wird, am besten lösen kann. Da bietet sich das theoretische Konstrukt des Schalenmodells an. Der Vorteil eines solchen Schalenmodells ist, dass es "generell [...] verschiedene Niveaus der Datenrelevanz und Inhaltserschließung“ zulässt, „die in einem gemeinsamen Informationssystem durch Transferkomponenten aufeinander zu beziehen sind. Ihnen kommt eine entscheidende Bedeutung zu. "122 In der bibliothekarischen Inhaltserschließung können diese Transferkomponenten z.B. Konkordanzen zwischen Klassifikationen und Thesauri ${ }^{123}$ sein.

Ausgangspunkt bei der Konstruktion eines solchen Schalenmodells ist, dass sich um einen Kern aus Daten mit inhaltlich hochwertiger Erschließung verschiedene Schichten aus Sacherschließungsdaten von immer geringerer Qualität bis hin zu Daten ohne jeglicher Erschließung befinden. Die Anzahl, die Merkmale und der Inhalt der angesetzten Schalen richtet sich ganz nach den jeweiligen Gegebenheiten. ${ }^{124}$ Das Retrieval ${ }^{125}$ in einem solchen Pool von heterogenen Daten muss dabei auf alle Dokumente zielen, die in Frage kommen. Der Sinn der Anwendung dieses Modells ist, dass durch den Kern der gut und ausführlich erschlossenen Dokumente z.B. durch statistische und probalistische Modelle ${ }^{126}$ auch diejenigen relevanten Dokumente aufgefunden werden, die weniger gut und ausführlich erschlossen sind, bzw. keinem Erschließungsprozess unterliefen.

Die verschiedenen Schichten könnten im Bezug auf die Erschließung von Altbeständen beispielhaft nach folgenden Qualitätskriterien eingeteilt werden, wobei es natürlich viele verschiedene Möglichkeiten der Einteilung gibt und diese vor einer Realisierung genau analysiert werden müssten. ${ }^{127}$

122 Krause, Jürgen; Schwänzl, Roland (1998)

123 „Ein Thesaurus im Bereich der Information und Dokumentation ist eine geordnete Zusammenstellung von Begriffen und ihren (vorwiegend natürlichsprachlichen) Bezeichnungen, die in einem Dokumentationsgebiet zum Indexieren, Speichern und Wiederauffinden dient" Vgl. Kuhlen, Rainer; Seeger, Thomas; Strauch, Dietmar (Hg.) (2004b): Grundlagen der praktischen Information und Dokumentation. Begr. von Klaus Laisiepen... Band 2: Glossar. München: Saur, hier S. 120; zitiert nach DIN 1463

${ }^{124}$ Krause, Jürgen (2004a): Standardisierung und Heterogenität. In: Kuhlen, Rainer; Seeger, Thomas; Strauch, Dietmar (Hg.): Grundlagen der praktischen Information und Dokumentation. Begr. von Klaus Laisiepen ... Band 1: Handbuch zur Einführung in die Informationswissenschaft und -praxis. München: Saur, S. 635-641, hier S.639

125 „Retrieval (auch Recherche oder Information Retrieval genannt) bezeichnet den Arbeitsvorgang des gezielten Suchens bzw. Wiederfindens von relevanten Daten und Fakten zu einer speziellen Fragestellung in gedruckten und elektronischen Informationsmitteln." Vgl. Kuhlen, Rainer; Seeger, Thomas; Strauch, Dietmar (Hg.) (2004b), hier S. 107

${ }^{126}$ Während andere beim Retrieval verwendete Modelle unterschiedliche Arten von Ähnlichkeiten zwischen der Suchanfrage des Nutzers und der Dokumentenbeschreibung berechnen, schätzen probalistische Modelle die Warscheinlichkeit, dass ein Dokument als relevant für den Nutzer betrachtet wird. Die Dokumente werden zudem nach dieser Wahrscheinlichkeit geordnet. Diese Ordnung nach Wahrscheinlichkeit nennt man Ranking. Vgl. Kuhlen, Rainer; Seeger, Thomas; Strauch, Dietmar (Hg.) (2004b), hier S. 99

$127 \mathrm{Vgl}$. dazu auch Stumpf, Gerhard (2002): Einführung in die verbale Inhaltserschließung für Bibliotheken. Kurzfassung des Skripts zum Kompaktkurs Juni 2002, S.2 


\begin{tabular}{|c|c|}
\hline ochwertige Erschließung & \\
\hline Schale 1 & $\begin{array}{l}\text { Altbestände mit syntaktischen Indexaten } \\
\text { (z.B. RSWK) / } \\
\text { RVK-Notation }\end{array}$ \\
\hline Schale 2 & $\begin{array}{l}\text { Altbestände mit kontrollierten Deskriptoren } \\
\text { (z.B. SWD) / } \\
\text { Altbestände mit Gattungsbegriffen / } \\
\text { Altbestände mit Historischen Klassifikationen }\end{array}$ \\
\hline Schale 3 & $\begin{array}{l}\text { Altbestände mit frei vergebenen Schlagwörtern / } \\
\text { Altbestände mit manuell angelegten Titelstichwörtern / } \\
\text { Altbestände mit angepassten und übersetzten Titel- } \\
\text { stichwörtern }\end{array}$ \\
\hline Schale 4 & Altbestände mit maschineller Indexierung \\
\hline Schale 5 & $\begin{array}{l}\text { Altbestände ohne Inhaltserschließung (nur die Titel- } \\
\text { stichwörter in ihrer ursprünglichen Form können zur } \\
\text { Recherche herangezogen werden) }\end{array}$ \\
\hline Keine Erschließung & \\
\hline
\end{tabular}

Da es sich bei diesen Überlegungen ja um alte Drucke handelt, die sich in ihrer Ausstattung bekanntlich von modernen Büchern unterscheiden, entfallen bei den Überlegungen zu einem geeigneten Schalenmodell Erschließungsdaten, die bei aktueller Literatur genutzt werden können. Dabei handelt es sich um Inhaltsverzeichnisse (wobei diese natürlich auch schon bei älteren Drucken teilweise vorhanden sind und somit evtl. auch bei diesen genutzt werden könnten), Abstracts, Klappentexte usw.

In Schale 1 werden natürlich die Drucke eingeteilt, die bestmöglich erschlossen sind. Diese Erschließung kann über syntaktische Indexate, z.B. Schlagwortketten, oder über eine moderne, tiefgehende Klassifikation erfolgen. Bei historischer Literatur im deutschen Sprachraum trifft dies natürlich vor allem auf die mit der RSWK und/oder den RVK erschlossenen Dokumente zu. Optimal ist es natürlich, wenn das Dokument sowohl verbal als auch klassifikatorisch erschlossen ist. Eventuell könnte diese Konstellation eine eigene Schale erhalten. Es ist aber auch durchaus denkbar, dass bei entsprechender Crosskonkordanz im deutschen Sprachraum eher selten genützte ErschlieBungsdaten in Form von z.B. der LCSH oder anderer tiefergehender Klassifikationen wie z.B. der Dewey Decimal Classification (DDC) in dieser Schale ihren Platz finden. Bei der Arbeit mit modernen Schlagwortverzeichnissen und modernen Klassifikationen 
muss jedoch immer bedacht werden, dass diese mit den alten Begrifflichkeiten nicht kompitabel sind.

In Schale 2 finden Dokumente Platz, die mit Hilfe kontrollierter Deskriptoren erschlossen wurden. So z.B. mit Schlagwörtern aus der Schlagwortnormdatei. Eine solche Erschließung ist zeitsparender als eine Erschließung mit Schlagwortketten und dabei beinahe so wirkungsvoll, jedoch werden die Verhältnisse, in welchem die verschiedenen Deskriptoren zueinander stehen, aus der Erschließung nicht ersichtlich. Dadurch kann es zu Unstimmigkeiten und zu einer fehlerhaften Recherche kommen. Auch Gattungsbegriffe können in diese Schale miteinbezogen werden, da ja auch sie normierte und kontrollierte Deskriptoren darstellen. Da Gattungsbegriffe in ihrer Anzahl jedoch meistens sehr begrenzt sind und daher eher grob erschließen, könnten sie auch in einer eigene Schale abgelegt werden. Ebenfalls mit in diese Schale könnten Dokumente, die eine Notation einer historischen Klassifikation aus einem alten Systematischen Katalog tragen. Mit Hilfe von Konkordanzen könnten diese für eine bibliotheksübergreifende Recherche genutzt werden. (s. Kap. 7.3.)

In Schale 3 kämen Dokumente, deren sachliche Erschließung mit frei vergebenen Schlagwörtern, bzw. mit manuell angelegten, angepassten oder übersetzten Titelstichwörtern durchgeführt wurde. Diese Formen der Erschließung sind natürlich weniger hochwertig als diejenigen mit Deskriptoren, jedoch wäre nach wie vor eine gezielte Recherche möglich.

Schale 4 beinhaltet Dokumente, deren Sacherschließung mit Hilfe maschineller Indexierung erfolgt, falls solche automatisch generierten Daten überhaupt mit einbezogen werden. Ist diese Methode zwar bei aktueller Literatur durchaus vielversprechend, so gibt es bei Altbeständen erhebliche Schwierigkeiten. Näheres zu dieser Technologie und den damit verbunden Problemen bezüglich Alter Drucke wird in Kap. 7.5. Automatische Inhaltserschließung erläutert.

In Schale 5 würden Drucke und Dokumente ihren Platz finden, die keinerlei Inhaltserschließung erfahren haben. Die einzige Möglichkeit, sie aufzufinden, besteht in der Recherche nach den formalen, in keinerlei Weise normierten oder angepassten Titelstichwörtern. 


\subsection{Suchmaschinentechnologie}

Eine große Chance für die inhaltliche Erschließung von Bibliotheksbeständen liegt in der Anwendung von Suchmaschinentechnologie in den elektronischen Katalogen der Bibliotheken. Dies gilt gerade auch für Altbestände. Diese Techniken werden bereits in einigen Verbünden, Projekten und sonstigen Katalogen angewandt. So z.B. auch im Dreiländerkatalog, der im folgenden näher betrachtet wird. Die Grundlage seiner Funktionsweise bildet die Technologie von Fast Data Search. ${ }^{128}$ Eine weitere Anwendung, welche auf der Basis derselben Technologie arbeitet, ist BASE. ${ }^{129}$

Durch die Übertragung der Besonderheiten und Funktionsweisen von Suchmaschinentechnologien auf Bibliothekskataloge können diese optimiert und optimal auf die Bedürfnisse der Nutzer zugeschnitten werden. Dies beginnt schon bei der einfachen Bedienung. So erscheint bei der einfachen Suche lediglich ein Feld zur Recherche in allen Kategorien. ${ }^{130}$ Sucht man nun nach einem Stichwort, bekommt man innerhalb einer sehr kurzen Bearbeitungszeit, in der die Suchmaschine binnen weniger Augenblicke einen riesigen Index auswertet, die Treffer in Form eines Relevance Ranking angeboten. Dies bedeutet, dass die gefundenen Dokumente automatisch nach ihrer Relevanz für den Nutzer eingeschätzt und auf Grundlage dieser Gewichtung entsprechend aufgelistet werden. Dabei entsteht ein homogener Index. Diese Rangbildung basiert auf voreingestellten Gewichtungen der einzelnen bibliographischen Felder. Wenn also der gesuchte Begriff bei dem ersten Titel im Hauptitel vorkommt und bei dem zweiten im Erscheinungsort, wird zuerst der erste Titel angezeigt, falls eine solche Gewichtung zuvor festgelegt wurde. Weitere Relevanzkriterien sind die Nähe der zu suchenden Terme zueinander und wie häufig ein bestimmter Begriff im gesamten Dokument vorkommt, wobei dies vor allem auf Volltexte wie z.B. Online-Dokumente zutrifft. Zusätzlich kann eingestellt werden, dass ähnliche Begriffe in die Suche einbezogen werden (Ähnlichkeitssuche oder Fuzzy Search). Wenn man sich bei der Eingabe vertippt, werden automatisch Rechtschreibvorschläge angezeigt. Dies funktioniert mittels Einbindung linguistischer Verfahren. Ebenfalls aufgrund dieser linguistischen Verfahren be-

${ }^{128}$ Köstädt, Peter (2006): FAST-Anwendungen und Dienstleistungen des hbz. Veranstaltung vom 31.05.06, aus der Reihe "Fast-Workshop". Köln. Veranstalter: hbz. Online verfügbar unter http://www.hbz-nrw.de/dokumentencenter/produkte/dlk/aktuell/vortraege/FAST-Workshop/FAST-Workshop_pk.pdf, zuletzt geprüft am 18.02.2008.

${ }^{129}$ BASE - Bielefeld Academic Search Engine (2008). Online verfügbar unter http://www.basesearch.net/, zuletzt geprüft am 18.02.2008. Vgl. zu dieser Thematik u.a. auch Lewandowski, Dirk (2006): Suchmaschinen als Konkurrenten der Bibliothekskataloge. Wie Bibliotheken ihre Angebote durch Suchmaschinentechnologie attraktiver und durch Öffnung für die allgemeinen Suchmaschinen populärer machen können. In: Zeitschrift für Bibliothekswesen und Bibliographie, Jg. 53, H. 2, S. 71-78. Online verfügbar unter http://www.durchdenken.de/lewandowski/doc/ZFBB_Preprint.pdf, zuletzt geprüft am 18.02.2008 sowie Lossau, Norbert (2004): Suchmaschinentechnologie und Digitale Bibliotheken - Bibliotheken müssen das wissenschaftliche Internet erschließen. In: Zeitschrift für Bibliothekswesen und Bibliographie, Jg. 51, H. 5-6, S. 284-294 und Gillitzer, Berthold; Weber, Andreas (2007): Des OPACs neue Kleider - vom Benutzerkatalog zur Suchmaschine. Neue Möglichkeiten für Bibliothekskataloge durch den Einsatz von Suchmaschinentechnologie. In: Bibliotheksforum Bayern, Jg. 1, H. 4, S. 218-221. Online verfügbar unter http://tinyurl.com/2hyqjq, zuletzt geprüft am 12.02.2008.

${ }^{130}$ Auch bekannt unter der Bezeichnung „Google-Schlitz“ 
steht die Möglichkeit der Wortstammbildung. Mit der Umkreissuche lassen sich die Treffer anhand der Postleitzahl auf ein bestimmtes Gebiet eingrenzen. Ein weiterer Vorteil der Suchmaschinentechnologie besteht in der Möglichkeit, gescannte Inhaltsverzeichnisse, Abstracts und weitere relevante Daten der jeweiligen Werke in die Titelanzeige einzubinden. Dies bezeichnet man als Catalogue Enrichment oder Kataloganreicherung, wobei dies bei alten Drucken hauptsächlich das Einscannen von Titelblättern und weiteren relevanten Seiten wie im VD 17 betrifft. So bringen die genannten Möglichkeiten zwar erhebliche Vorteile für den Nutzer bei der Recherche mit sich. Bezogen auf die inhaltliche Altbestandserschließung sind sie jedoch eher nebensächlich. Ein großer Vorteil dieser neuen Technologien besteht hingegen darin, dass sie weitere Funktionen besitzen, mit deren Hilfe man auch die Sacherschließung von Altbeständen vorantreiben kann. So ist darin das Drill-Down-Verfahren integriert, womit man die nach der erfolgreichen Suche angezeigten Treffer kategorisieren kann, z.B. nach Sprache, Themen (Schlagwörter), Publikationstypen und, in diesem Kontext besonders interessant, nach Jahren. So lassen sich Trefferlisten auf das gewünschte Maß reduzieren und man kann gezielt auf ältere Literatur eingrenzen. Ähnliches ermöglicht die Universitätsbibliothek Bayreuth mit der Suche in inrem InfoGuide. ${ }^{131}$ Dieser basiert ebenfalls auf der FAST-Technologie und lässt den Nutzer seine Trefferliste nicht nur nach Jahr, Sprache, Publikationstyp usw. eingrenzen, sondern bietet auch die Gelegenheit, diese nach Schlagwortketten zu filtern. Eine weitere bedeutende Funktionsweise, gerade in Anbetracht der Anwendung des Schalenmodells in der Altbestandserschließung, ist die Möglichkeit einer virtuellen Clusterung von Nachweisen eines Titels aus verschiedenen Bibliotheken. Dieser Vorgang ist auch unter der Bezeichnung Virtuelle Dublettenzusammenführung bekannt. Es werden automatisch gleiche Werke erkannt und diese in der Ergebnissanzeige im Anschluss an die Recherche zusammengefasst und unter einer einzigen Titelanzeige angegeben. Durch diese Dublettenzusammenführung genügt bereits die qualitativ gute inhaltliche Erschließung an einer Bibliothek, um mit Hilfe dieser Verschlagwortung sämtliche selben Titel aufzufinden, völlig unabhängig von deren Erschließungszustand. Diese Form der Dublettenbehandlung wird auch Match- und Merge-Verfahren genannt. Dieses Verfahren ist also somit „in der Lage, Katalogisate aus verschiedenen lokalen Bibliotheken, die identische Bücher und Dokumente bezeichnen, auch als identisch zu erkennen (match) und unter einem einzigen, gemeinsamem Nachweis in der Suchmaschine zu verzeichnen (merge). “132

${ }^{131}$ InfoGuide UB Bayreuth. (2008) Online verfügbar unter http://opac.uni-bayreuth.de:8080/InfoGuideClient/initSearch.do, zuletzt geprüft am 18.02.2008. Ein weiterer OPAC, der die Eingrenzung nach Schlagwörtern und Jahren emöglicht, ist der OACplus der Bayerischen Staatsbibliothek. OPACplus. Bayerische Staatsbibliothek (2008). Online verfügbar unter https://opacplus.bsb-muenchen.de/InfoGuideClient/start.do?Login=opacext\&BaseURL， zuletzt geprüft am 18.02.2008.

132 Lügger, Joachim (2000): Über Suchmaschinen, Verbünde und die Integration von Informationsangeboten. Teil I. KOBV-Suchmaschine und Math-Net. Konrad-Zuse-Zentrum für Informationstechnik Berlin. (ZIB-Report, 00-23). Online verfügbar unter http://www.zib.de/Publications/Reports/ZR-00-23.pdf, zuletzt geprüft am 18.02.2008, hier S. 11. Eine genaue Erläuterung des Match- und Merge-Verfahrens, ebenfalls bezogen auf den KOBV, dessen Verbundkatalog ebenso auf Suchmaschinentechnologie basiert, findet statt in Kuberek, Monika (1999): Dublettenbehandlung (Match- und Merge-Verfahren) in der KOBV-Suchmaschine. Grundlagen. Konrad-Zuse-Zentrum für Informationstechnik Berlin. (Preprint, SC 99-16). Onli- 
Diese Technologie bedeutet natürlich auch in der Altbestandsinhaltserschließung eine enorme Arbeitsersparnis und schafft zusammen mit den anderen genannten Punkten im Allgemeinen und speziell für zukünftige Kooperationen zwischen Bibliotheken und Verbünden gänzlich neue Möglichkeiten. ${ }^{133}$

\subsection{Konkordanzen}

Da eine durchgängige rückwärtige Beschlagwortung nach einem heute gängigen Regelwerk, wie z.B. den RSWK oder den LCSH, vom Aufwand und von den Kosten warscheinlich viel zu groß wäre und eine Realisierung deswegen eher unwarscheinlich ist, sollte versucht werden, auf bereits vorhandene Daten zurückzugreifen, die in der Vergangenheit unter Einsatz von viel Energie und mit großen Kosten angefertigt wurden. „In Dresden hatten“ z.B. „16 wissenschaftliche Mitarbeiter während 12 Jahren zwischen 1866 und 1878 den systematischen Standortkatalog der Landesbibliothek erarbeitet, der bis 1926 weitergeführt wurde. "134 Grundlegend bedeutet dies, dass Konkordan$z{ }^{135}$ zwischen historischen Klassifikationen und Deskriptoren geschaffen werden sollten, um dem Nutzer verbale, deutschsprachige Sucheinstiege zu ermöglichen. „Crosskonkordanzen sind gerichtete, relevanzbewertete Relationen zwischen Termen zweier Thesauri, Klassifikationen oder auch anderer kontrollierter Vokabulare. Die Erstellung der Relationen erfolgt intellektuell. “136 Generell werden Konkordanzen entwickelt, um „Vor dem Hintergrund der durch das WWW hervorgerufenen Möglichkeiten zur Vernetzung von Informationssystemen [...] eine integrierte Suche nach inhaltlichen Gesichtspunkten in verteilten, heterogen erschlossenen Datenbeständen“ zu ermöglichen. Dabei wird eine Dokumentationssprache auf eine andere abgebildet. „Die Grundidee dabei ist, mittels einer einzigen Dokumentationssprache auf Dokumentbestände zuzugreifen, die mit unterschiedlichen Dokumentationssprachen entwickelt wurden." ${ }^{137}$ Da man bei der Erschließung von Altbeständen in gleicher Weise auf einen heterogenen Datenbestand zurückgreifen muss, können diese Konkordanzen ebenso zwischen historischen und/oder modernen Klassifikationen geschaffen werden. Dazu ist die Konversion von alten Systematischen Katalogen notwendig. Bürger plädiert in seinem Aufsatz

ne verfügbar unter http://www.zib.de/Publications/Reports/SC-99-16.pdf, zuletzt geprüft am 18.02.2008.

133 Zu den Möglichkeiten des Dreiländerkatalogs siehe auch Dreiländerkatalog (2006) und Weber, Andreas (2007): Suchmaschinentechnologie (FAST): Neue Möglichkeiten für den Benutzer. Veranstaltung vom 14.12.2007. München. Online verfügbar unter http://www.bib-bvb.de/vk2006/FAST_Weber_Verkundk_2006.pdf, zuletzt geprüft am 18.02.2008.

134 Dimitriadu, Valentina (1994): Der historische Standortkatalog und der Fachkatalog der Sächsischen Landesbibliothek Dresden. Diplomarbeit. Dresden, S.20; zitiert nach: Bürger, Thomas (2004), hier S. 89

135 Neben dem Begriff „Konkordanz“ ist auch der Begriff „Crosskonkordanz“ gebräuchlich

136 Mayr, Philipp; Walter, Anne-Kathrin (2007): Zum Stand der Heterogenitätsbehandlung in vascoda: Bestandsaufnahme und Ausblick. GESIS / Informationszentrum Sozialwissenschaften (IZ). Bonn. Online verfügbar unter http://www.ib.hu-berlin.de/ mayr/arbeiten/BID2007-Mayr-Walter.pdf, zuletzt geprüft am 18.02.2008, hier S. 3

137 Bertram, Jutta (2005): Einführung in die inhaltliche Erschließung. Grundlagen - Methoden Instrumente. Würzburg: Ergon-Verl. (Content and communication, 2), hier S. 253 
„Historische Drucke in Virtuellen Fachbibliotheken? Nutzen und Möglichkeiten sachlicher Sucheinstiege in das VD 17 und VD 18“138 für die Anwendung solcher Konkordanzen speziell im VD 17 und VD 18. Jedoch sind diese Überlegungen auch allgemein auf Altbestände übertragbar. So sollten „die sprechenden Signaturen historischer Aufstellungsordnungen transparenter und komfortabler recherchierbar gemacht werden. “139 $\mathrm{Er}$ geht dabei von dem Standpunkt aus, dass vor der Einführung des Numerus currens die Literatur in den Bibliotheken zwischen dem 17. und dem 19./20. Jahrhundert in systematischen Aufstellungen geordnet waren, z.B. so wie bereits genannt in Form von Realkatalogen wie in der StaBi Berlin und in der SUB Göttingen. So ist es bereits in mehreren Bibliotheken möglich, über ihre jeweilige Internetpräsenz einen sachlichen Zugang zu den systematisch geordneten Altbeständen zu erlangen. ${ }^{140}$ Weiter plädiert Bürger für „die Verlinkung des VD 17 mit den Übersichten über die Aufstellungssysteme der beteiligten Bibliotheken." 141 Dadurch würde es dem interessierten Wissenschaftler ermöglicht, über die vorhandenen Gattungsbegriffe hinaus spezifische Altbestände der jeweiligen Bibliotheken zu ermitteln. „Eine Recherche in systematischen Katalogen führt in der Regel über die modernen Fachgruppenbezeichnungen zu den historischen Fachgruppen, die je nach Bestandsgröße vielfach unterteilt und mit entsprechenden Aufstellungssystematiken gekennzeichnet sind." ${ }^{142}$ So können mit Hilfe dieser sprechenden Signaturen Rechercheergebnisse erzielt werden, die ansonsten nicht möglich wären.

Bestrebungen in dieser Richtung bestehen z.B. auch in der UB Freiburg, in der man sich ebenfalls mit Möglichkeiten der klassifikatorischen Erschließung als Ergänzung zur verbalen Sacherschließung beschäftigt. Die UB Freiburg verzeichnet ihren Altbestand sachlich in einem systematischen Katalog 1500 -1967, in dem die Bestände nach einer leicht modifizierten Version des Hartwigschen System in 23 Sachgruppen, die wieder-

${ }^{138}$ Vgl. Bürger, Thomas (2004)

${ }^{139}$ Vgl. Bürger, Thomas (2004), hier S. 91

${ }^{140}$ Siehe u.a.: Der Alte Realkatalog der Bayerischen Staatsbibliothek: ARK-Online (2008). Online verfügbar unter http://arkat.bsb-muenchen.de/start.php?offen=@@RK@@\&sprung $=$ RK\&sy=RK\&modus=PUB, zuletzt geprüft am 18.02.2008.

Der Image-Katalog des Hartwig Katalogs der ULB Sachsen-Anhalt Halle: ULB Halle: ImageKataloge (2001). Online verfügbar unter http://zkat.bibliothek.uni-halle.de/, zuletzt aktualisiert am 26. 04. 2001, zuletzt geprüft am 18.02.2008.

Der ARK Sachkatalog (1501-1955) der Staatsbibliothek zu Berlin: ARK Sachkatalog der Staatsbibliothek zu Berlin - Preußischer Kulturbesitz (2008). Online verfügbar unter http://ark.staatsbibliothek-berlin.de/index.php, zuletzt geprüft am 17.02.2008.

Der Systematische Standortkatalog der Staats- und Universitätsbibliothek Dresden: Systematischer Standortkatalog SLUB (2003). Online verfügbar unter http://image.slub-dresden.de/de/lnc_txt/stok/index.html, zuletzt aktualisiert am 16.11.2003, zuletzt geprüft am 18.02.2008.

Der Band-Realkatalog der SUB Göttingen: Niedersächsische Staats- und Universitätsbibliothek (2008). bandrksyst. Online verfügbar unter http://opac.sub.unigoettingen.de/DB=1/BANDRKSYST, zuletzt geprüft am 17.02.2008.

Der digitalisierte Systematische Katalog der Württembergischen Landesbibliothek (DigiSyk): DigiSyk-Benutzerversion (2008). Online verfügbar unter http://digisykben.wlbstuttgart.de:8080/digisyk2ben /ben_syk_funktionen.php?indexsuche $=1 \&$ syk_root_id $=10$, zuletzt geprüft am 18.02.2008.

${ }^{141}$ Bürger, Thomas (2004), hier S. 92

142 Bürger, Thomas (2004), hier ebenfalls S. 92 
um weiter untergliedert sind, festgehalten werden. Um eine verbesserte Darstellung und Abrufbarkeit der Lesesaalbestände und anderer Sonderstandorte zu ermöglichen, wurde ein System entwickelt, welches auf dem Standortkatalog beruht und es ermöglicht, die Literatur systematisch aus dem Online-Katalog abzurufen. Seit einiger Zeit sind dazu Überlegungen im Gange, wie diese Technik auf die Altbestandssystematik übertragen werden kann. ${ }^{143}$ Die Altbestandssystematik ist online abrufbar und es lässt sich per Systematikstichwort und Signatur darin recherchieren. ${ }^{144}$

Obwohl eine mögliche Realisierung durchaus skeptisch betrachtet wird (vgl. u.a. Kap. 4.5.), müssen für die Zukunft Lösungen dafür gefunden werden, Konkordanzen für die unterschiedlichen historischen Signaturgruppen und die entsprechenden verbalen Sucheinstiege zu entwickeln. So sollte es ermöglicht werden, z.B. über die Eingabe ein oder mehrerer normierter Deskriptoren wie etwa Skandinavien und Geschichte im Dreiländerkatalog direkt auf die Literatur, die innerhalb der Systematikgruppe IV.18 (Geschichte der skandinavischen Reiche) in der Öttingen-Wallersteinschen-Bibliothek der UB Augsburg verzeichnet ist, zu gelangen.

Die Methodik von Crosskonkoranzen zwischen Thesauri und Klassifikationen wurde im Projekt CARMEN entwickelt. Ausgangspunkt der Überlegungen war, dass in Bibliotheken und Fachinformationssystemen unterschiedliche Klassifikationen und Thesauri verwendet werden und dadurch eine fach- und datenbankübergreifende Recherche deulich erschwert wird. Eine effiziente Suche ist kaum möglich, da man bei der Benutzung verschiedener Bibliothekskataloge und Datenbanken jeweils mit eigenen Suchbegriffen und einer eigenen Suchlogik arbeiten muss. Ziel des Projektes war deswegen die Ermöglichung einer integrierten Suche nach sachlichen Gesichtspunkten mit unterschiedlichen inhaltlichen Schwerpunkten in verteilten Datenbeständen. Die begrifflichen Unterschiede der verwendeten Thesauri und Klassifikationen sollen durch die Entwicklung von Crosskonkordanzen dabei überwunden werden. ${ }^{145}$ „Auf diesem Weg können auf einer etwas gröberen Erschließungsebene, Brücken zwischen großen, intensiv erschlossenen Mengen von Dokumenten und unter Umständen fremden Suchumgebungen mit Online-Klassifikationen gebaut werden. "146 Konkret realisiert werden soll dies für die RVK und die DDC. ${ }^{147}$

${ }^{143}$ Reifegerste, Matthias (2007): Der systematische Katalog des Altbestandes $1500-1967$. Überarbeitete Fassung des Vortrags "Neues zum alten Freiburger SyKa 1500-1967. Eine Projektskizze". 31. Jahrestagung der Gesellschaft für Klassifikation. Veranstaltung vom 07.03.2007. Freiburg.

${ }^{144}$ UB-Freiburg Systematiken (2008). Online verfügbar unter http://matterhorn-ng.ub.uni-freiburg.de/SystWebS/systematik-webservice.php?ctrl=showroot\&thessi=AB\&signatur=\&css=css3, zuletzt geprüft am 18.02.2008.

145 Vgl. CARMEN AP12 (2000). Crosskonkordanzen von Klassifikationen und Thesauri. Online verfügbar unter http://www.bibliothek.uni-regensburg.de/projects/carmen12/index.html.de, zuletzt aktualisiert am 18.02.2000, zuletzt geprüft am 18.02.2008.

${ }^{146}$ Stumpf, Gerhard (2002): Online-Klassifikation und Klassifikation im Online-Katalog - Alternativen für die RVK? 92. Deutscher Bibliothekartag. Veranstaltung vom 12.04.2002. Augsburg. Online verfügbar unter http://www2.bibliothek.uni-augsburg.de/allg/swk/Online-Klassifikation.html, zuletzt geprüft am 18.02.2008.

147 Vgl. CARMEN AP12 (2000) und Bertram, Jutta (2005), hier S. 254 
Ein Beispiel einer solchen Crosskonkordanz zwischen Schlagwort und Klassifikation ist die Entwicklung einer Konkordanz zwischen der SWD und der DDC an der Deutschen Nationalbibliothek im Zuge des Projektes CrissCross. Innerhalb dieses Projektes wird die Herstellung einer Verbindung zwischen den ca. 150.000 Sachschlagwörtern der SWD und der DDC angestrebt. ${ }^{148}$

Es gilt, diese Entwicklungen auch für die Altbestandserschließung zu nutzen.

\subsection{Kooperationen}

Durch Kooperationen verschiedener Bibliotheken und Verbünde entsteht die Möglichkeit einer weit effizienteren arbeitsteiligen Erschließung. Diese könnte z.B. ebenfalls im Dreiländerkatalog verwirklicht werden. Wenn dieser die Bestände aller Bibliotheken des deutschsprachigen Raumes verzeichnet und die Bibliotheken ihre Daten weiterhin permanent einspielen werden, dann wird diese wesentlich effizientere arbeitsteilige Erschließung möglich. Als Grundlage können die Daten der großen Altbestandsbibliotheken wie diejenigen der StaBi Berlin und der BSB herangezogen werden. Dabei sollte eine engere Zusammenarbeit in diesem Bereich zwischen den einzelnen Bibliotheken erwogen und realisiert werden, ähnlich der bereits bestehenden Zusammenarbeit im VD 17.

Um diese Zusammenarbeit zu erleichtern und möglichst zu automatisieren, sollte das Konzept der Kooperativen Neukatalogisierung umgesetzt werden. ${ }^{149}$ Dieses Konzept geht von dem Standpunkt aus, dass durch die Verbundstruktur des deutschen Bibliothekswesens zahlreiche Titel mehrfach erfasst werden, da die Eigenkatalogisierungsquote in den einzelnen regionalen Verbünden relativ hoch ist und es selten ist, dass bestimmter Bestand nur in einem Verbund vorhanden ist. Durch die Entwicklung eines solchen Konzeptes soll eine Verbesserung des Fremddatenangebotes im eigenen Verbund durch die Einbeziehung anderer Verbunddatenbanken erreicht werden. Dies beinhaltet, dass schon bei der Erwerbungskatalogisierung Titeldaten übernommen

${ }^{148}$ Bee, Guido; Karg, Helga (2007): Navigieren zwischen Schlagwort und Notation. CrissCross als Verbindung zwischen SWD und DDC. 96. Deutscher Bibliothekartag 2007. Veranstaltung vom 19.03.2007. Leipzig. Online verfügbar unter http://www.opus-bayern.de/bib-info/volltexte/2007/381/pdf/LeipzigVortragstext.pdf, zuletzt geprüft am 18.02.2008.

149 Vgl. Block, Barbara (2006): Kooperative Neukatalogisierung. Eine neue Facette in der Zusammenarbeit der Verbünde. 95. Deutscher Bibliothekartag 2006. Veranstaltung vom 22.03.2006. Dresden. Online verfügbar unter http://www.gbv.de/vgm/info/biblio/01VZG/06Publikationen/2006/pdf/pdf_1806.pdf, zuletzt geprüft am 18.02.2008 und Block, Barbara (2007): Kooperative Neukatalogisierung als Standardverfahren in der verbundübergreifenden Zusammenarbeit. 96. Deutscher Bibliothekartag 2007. Veranstaltung vom 20.03.2007. Leipzig. Online verfügbar unter https://www.gbv.de/vgm/info/biblio/01VZG/06Publikationen/2007/pdf/pdf_2853.pdf, zuletzt geprüft am 18.02.2008. Zum selben Thema siehe Block, Barbara; Labner, Josef; Rusch, Beate (2007): Katalogisierung kooperativ gemacht. In: Bibliotheksdienst, Jg. 41, H. 2, S. 150-158. Online verfügbar unter http://www.zlb.de/aktivitaeten/bd_neu/heftinhalte2007/BD_Erschliessung_01_0207.pdf, zuletzt geprüft am 18.02.2008. Aus diesem Artikel stammt auch die folgende Feststellung (S. 152): „Für den Bereich der Sacherschließung wurden noch keine Festlegungen getroffen, da der Arbeitsschwerpunkt zunächst bei den Indices lag, die für die Formalkatalogisierung benötigt werden. Festlegungen für den Bereich der Sacherschließung sollen zu einem späteren Zeitpunkt ergänzt werden." 
werden. Diese Titeldaten sind zusätzlich angereichert mit Sacherschließungsdaten, also mit Schlagwörtern und mit Notationen. Der Austausch der Daten sollte täglich stattfinden, und zwar in automatisierter Form. Dabei muss festgelegt werden, welches Verfahren der Datenübernahme angewendet werden soll, welches Austauschformat benutzt werden soll und natürlich auch, auf welchen Regeln die Katalogisierung beruht. Ebenfalls muss festgelegt werden, welche Art der Sacherschließung man anwendet. Bei den Schlagwörtern dürften dies die RSWK sein, wobei man gerade im Bereich der Altbestandserschließung natürlich auch die abgestufte Inhaltserschließung nach Art des Schalenmodells, also auch mit Stichwörtern, Gattungsbegriffen usw. miteinbeziehen sollte. Zudem könnte gerade dort die Anwendung von Konkordanzen eine gewichtige Rolle spielen. Bei der Klassifikation gibt es hingegen mehrere Möglichkeiten. Am ehesten in Frage käme aufgrund ihrer Verbreitung und ihrer Anwendung im Dreiländerkatalog die RVK.

Somit sollten auf der Basis des Schalenmodells, der Nutzung von Konkordanzen, den neuen Möglichkeiten des Datenaustausches und den neuen Technologien in Bibliothekskatalogen wie der Virtuellen Dublettenzusammenführung auch in der Altbestandserschließung neue Möglichkeiten entstehen, diese mit Hilfe von Kooperationen zwischen den Verbünden und zwischen Bibliotheken im finanziell und personell machbaren Rahmen durchzuführen.

\subsection{Automatische Inhaltserschließung}

Wie bereits mehrfach in dieser Arbeit angesprochen, liegt die mangelnde inhaltliche Erschließung von Altbeständen an dem enormen Aufwand und den damit verbundenen finanziellen und personellen Nachteilen. Der Hauptgrund hierfür ist, dass eine solche Inhaltserschließung intellektuell durchgeführt werden muss. In den letzten Jahren gibt es deswegen zahlreiche Bestrebungen, Sacherschließung nicht mehr nur manuell, sondern auch maschinell, mittels automatischer Inhaltserschließung, bzw. Indexierung zu betreiben. „Automatische Indexierung ist die Indexierungsmethode, nach der zu einem Dokument Deskriptoren oder Notationen von einem Computer ermittelt werden. “150

Darunter „werden Verfahren verstanden, die vollautomatisch Dokumente analysieren und abgeleitet aus dieser Analyse entweder ausgewählte Terme aus dem Dokument extrahieren [...] und als Indexterme abspeichern [...] oder Deskriptoren einer kontrollierten Indexierungssprache dem Dokument als Inhaltsrepräsentanten zuweisen." ${ }^{151}$ Dabei haben sich vier verschiedene Verfahren durchgesetzt:

1. Einfache Stichwortextraktion (Volltext-, Freitextinvertierung)

2. Statistische Verfahren

3. Computerlinguistische Verfahren

\footnotetext{
${ }^{150}$ Nohr, Holger (2005): Grundlagen der automatischen Indexierung. Ein Lehrbuch. 3. Auflage. Berlin: Logos-Verl., hier S. 28

151 Kuhlen, Rainer; Seeger, Thomas; Strauch, Dietmar (Hg.) (2004b), hier S. 7
} 


\section{Begriffsorientierte Verfahren}

Obwohl das Verfahren der einfachen Stichwortextraktion „vollautomatisch arbeitet, indem es alle Wörter (im Sinne von Zeichenketten) eines Textes in einer invertierten Liste ablegt, kann es dennoch nicht eigentlich zu den automatischen Indexierungsverfahren gezählt werden, da bei diesen doch wenigstens eine Auswahl- und/oder Bearbeitungsfunktion erwartet würde." 152 Somit ist es mit dieser Methode nicht möglich, terminologisch kontrollierte Begriffe zu erzeugen, da die extrahierten Stichwörter weder analysiert noch weiterbehandelt werden.

Im Gegensatz dazu versuchen Statistische Verfahren „anhand der Häufigkeit des Auftretens von Begriffen in Texteinheiten Rückschlüsse auf ihre inhaltliche bzw. strukturelle Bedeutung für diese Texteinheiten zu ziehen. [...] Je häufiger ein Term in einem Dokument und je seltener er in der gesamten Dokumentensammlung auftritt, desto größer ist in der Regel sein konkreter Bezug zum Inhalt des Dokuments und desto eher ist er deshalb als Indexterm geeignet." ${ }^{153}$

Betrachten statistische Verfahren verschiedene Schreibweisen oder Flexionsformen ein und desselben Wortes als verschiedene Terme, können computerlinguistische Verfahren die Daten auf sprachgrammatischer Ebene bearbeiten. Auf den Dokumenteninhalt wird nicht lediglich aufgrund der Häufigkeitsverteilung von Begriffen geschlossen, sondern die Texteinheiten werden morphologisch, lexikalisch und syntaktisch behandelt. Dies bedeutet, dass die einzelnen Wörter des Dokuments isoliert und auf ihre Grund- bzw. Stammformen zurückgeführt werden. U.a. werden Phrasen erkannt und Komposita semantisch zerlegt. Als Ergebnis entsteht eine normalisierte Terminologie, die den Inhalt des analysierten Dokuments erkennbar werden lässt. ${ }^{154}$

Bei der vierten Methode, den begriffsorientierten Verfahren, kommt die Einbindung einer kontrollierten Indexierungssprache hinzu. Diese Methoden „abstrahieren von der gegebenen Wortwahl auf die Bedeutung der Texte. Die erkannte Bedeutung (der Inhalt) eines Dokuments wird anschließend auf eine kontrollierte Indexierungssprache abgebildet und durch deren Deskriptoren repräsentiert." ${ }^{155}$ Diese kontrollierte Indexierungssprache kann z.B. ein Thesaurus sein. So ist es z.B. bei diesem Verfahren möglich, synonyme Begriffe zusammenzuführen und Homonyme zu unterscheiden. Die anderen aufgezeigten Methoden leisten dies nicht. ${ }^{156}$

$\mathrm{Zu}$ unterscheiden ist außerdem zwischen automatischer Indexierung, worauf sich die bisherige Übersicht bezieht, und automatischer Klassifizierung. Automatische Klassifi-

\footnotetext{
152 Nohr, Holger (2005), hier S. 37

${ }^{153}$ Mittelbach, Jens; Probst, Michaela (2006), hier S. 171

${ }^{154}$ Mittelbach, Jens; Probst, Michaela (2006), hier ebenfalls S.171

${ }^{155}$ Nohr, Holger (2004): Theorie des Information Retrieval II: Automatische Indexierung. In: Kuhlen, Rainer; Seeger, Thomas; Strauch, Dietmar (Hg.): Grundlagen der praktischen Information und Dokumentation. Begr. von Klaus Laisiepen ... Band 1: Handbuch zur Einführung in die Informationswissenschaft und -praxis. München: Saur, S. 215-225, hier S. 223

${ }^{156}$ Mittelbach, Jens; Probst, Michaela (2006), hier ebenfalls S.171
} 
zierung bedeutet die Klassifizierung von Objekten oder Dokumenten mit maschinellen Mitteln. ${ }^{157}$

Werden automatische Verfahren zur Inhaltserschließung bei aktuellen Werken zwar verstärkt genutzt und sind vor allem bei digitalen Dokumenten sehr hilfreich und ideal anzuwenden, stellt sich doch bei Altbeständen die Frage, ob diese Methoden auch bei innen zu einer rationelleren Sacherschließung führen können. Dies muss eher verneint werden. Ein grosses Problem bei alten Drucken ist, dass diese Drucke häufig kein Inhaltsverzeichnis besitzen, es keinen Klappentext und auch keine Abstracts gibt. Teilweise wäre die Anwendung linguistischer und begriffsorientierter Verfahren möglich. So z.B. wenn die Titel des Werkes aussagekräftig und nicht in älterer deutscher Orthographie verfasst sind. Abzuwarten bleibt, wie in Kap. 3.2.3 beschrieben, welche Erkenntnisse die Einbeziehung von Digitalisaten in das VD 18 bringt. Ob es vom Aufwand her möglich sein wird, genügend Drucke zu digitalisieren, damit die Anzahl relevant wird, und diese dann als digitale Volltexte mittels OCR-Software durchsuchbar zu machen. Dies könnte Maschinelle Indexierung auch bei Altbeständen ermöglichen, wobei natürlich auch hier das Problem der alten Schreibweisen und Grammatiken bestehen bleibt.

157 Vgl. zu der Thematik des automatischen Klassifizierens u.a. Oberhauser, Otto (2005): Automatisches Klassifizieren und Bibliothekskataloge. In: Hrusa, Hans (Hg.): Bibliothek, Technik, Recht. Festschrift für Peter Kubalek zum 60. Geburtstag. Wien: Manz, S. 119-131 und Oberhauser, Otto; Gödert, Winfried (2005): Automatisches Klassifizieren. Entwicklungsstand - Methodik - Anwendungsbereiche. Mt einem Vorwort von Winfried Gödert. Frankfurt am Main: Lang (Europäische Hochschulschriften : Reihe XLI ; Informatik, 43). 


\section{Zusammenfassung und Ausblick}

Der allgemein bestehende Eindruck, dass Sacherschließung von Altbeständen in deutschen Bibliotheken zu großen Teilen weit unterentwickelt ist, kann nach Betrachtung der in dieser Arbeit angeführten Zahlen als zutreffend bezeichnet werden. Es existieren in diesem Bereich erhebliche Defizite. So ist nur ein geringer Teil der Altbestände inhaltlich erschlossen, dazu häufig nur unzureichend. Als Gründe für diese Situation werden immer wieder dieselben Ursachen angeführt: die hohen Kosten, der hohe Aufwand und die fehlenden Mittel und Mitarbeiter, um eine qualitätvolle sachliche Erschließung zu erbringen. Jedoch gibt es auch Ausnahmen, so z.B. die Österreichische Nationalbibliothek mit ihrer übergreifenden Erschließung nach den RSWK oder die untersuchten deutschen Altbestandsbibliotheken wie die Staatsbibliothek zu Berlin und die SUB Göttingen mit ihren historischen Systematiken. Die Bestände dieser Bibliotheken sind auf Grundlage dieser Systeme durchgehend klassifiziert. Leider ermöglichen solche historischen Systematiken nur eine lokale und keine überregionale Recherche. Das Problem mit der Terminologie vergangener Jahrhunderte und ihrer Kompatibilität mit modernen Begriffen ist eine weitere Schwierigkeit, auf die immer wieder hingewiesen wird.

Andererseits lässt sich deutlich erkennen, dass es für Bibliotheken durchaus lohnenswert ist, für einen sachlichen Zugang bei Altbeständen zu sorgen, da die bereits erschlossenen historischen Bestände auch intensiv genutzt werden, so z.B. in der Herzog August Bibliothek in Wolfenbüttel.

Wie sollte eine zukünftige Altbestandserschließung nun aussehen? Eine allgemeine Retrokatalogisierung nach den RSWK sollte wohl nur für Teilbestände angestrebt werden, da eine generelle Verschlagwortung aller Bestände wohl nicht möglich ist. Es sollten vielmehr die historischen systematischen Kataloge genutzt werden, so wie dies in Kap. 7.3. erläutert wurde. Wie gesehen besitzen gerade diejenigen Bibliotheken, die einen reichen Altbestand verzeichnen, häufig auch einen durchgängigen alten Systematischen Katalog. Dies trifft z.B. auf die StaBi Berlin, die SUB Göttingen und die Bayerische Staatsbibliothek zu. ${ }^{158}$ Diesen Umstand sollte man nutzen. Da der normale Bibliotheksnutzer erfahrungsgemäß wesentlich lieber verbal als klassifikatorisch sucht, sollte die erste Anstrengung darin liegen, verbale Sucheinstiege zu schaffen, bzw. diese auszubauen.

Zudem sollte versucht werden, neuartige Entwicklungen in der Technik wie z.B. die Einführung der Suchmaschinentechnologie in die Bibliothekskataloge und neue Bestrebungen wie das Konzept der Kooperativen Neukatalogisierung auch für die Altbestand-

${ }^{158}$ Neben denen in dieser Arbeit bereits erwähnten gibt es im deutschsprachigen Raum noch zahlreiche weitere solcher Kataloge. So z.B. in Oldenburg. Vgl. dazu Fietz, Rudolf (1992): Der alte Realkatalog der Landesbibliothek Oldenburg. Seine Entstehung und seine Systematik. In: Koolman, Egbert (Hg.): Ex Bibliotheca Oldenburgensi. Bibliothekarische Untersuchungen aus Anlaß des 200jährigen Bestehens der Landesbibliothek Oldenburg. Hrsg. von Egbert Koolman. Oldenburg: Holzberg (Schriften der Landesbibliothek Oldenburg, 26). 
serschließung nutzbar zu machen, und nicht immer nur die aktuelle Literaur und in zunehmenden Maße Onlinedokumente im Blickpunkt zu haben.

Zudem sollte überlegt werden, ob man bei Neuerschließungen oder Retrokatalogisierungen als Klassifikation nicht die Basisklassifikation nutzen sollte, so wie z.B. der GBV oder die ÖNB dies bereits handhaben; oder ob man nicht auch eher die RVK zur Altbestandserschließung heranziehen sollte. Dass sie für diese Aufgabe nicht absolut ungeeignet sein kann, zeigt z.B. ihre Anwendung in der UB Eichstätt. Eine weitere Möglichkeit wäre noch die DDC, obwohl doch mehrere Gründe dagegen sprechen, u.a. ihre mangelnde Verbreitung. Neue Erkenntnisse in diesem Bereich wird wohl auch eine Realisierung des VD 18 bringen. Dabei wird zu beobachten sein, welche Möglichkeiten der inhaltlichen Recherche darin angewandt werden.

Insgesamt kann festgestellt werden, dass es zu dieser Thematik noch zahlreiche weitere Facetten gibt, die man ebenso hätte heranziehen und betrachten können. In andere Teilaspekte wiederum hätte man noch tiefer eindringen und diese evtl. weit mehr ausbauen können. Für diese Arbeit musste jedoch eine Auswahl getroffen werden.

Abschließend sei noch gesagt, dass, „wie dem auch sei, niemand [...] ernsthaft den Nutzen bestreiten können“ wird, „den die Sacherschließung der alten Bücher den Wissenschaftsdisziplinen bieten würde. "159

${ }^{159}$ Arnold, Werner (1997), hier S. 334 


\section{Anhang: Fragen an Bibliotheken}

1. Erschließen Sie den Altbestand (aus den Jahren 1501-1850) Ihrer Bibliothek oder Teile des Altbestandes Ihrer Bibliothek inhaltlich?

2. Wenn ja: Wie gehen Sie dabei vor, wie führen Sie die Erschließung durch?

(z.B. Komplette Erschließung; Erschließung nur bei Neuanschaffungen; Erschließung in Form eines oder mehrerer Projekte; sukzessive Erschließung; etc.)

Erläutern Sie bitte kurz.

3. Wenn ja: In welcher Form erschließen Sie inhaltlich?

(z.B. Moderne Klassifikation; Historische (hauseigene) Klassifikation; RSWK; nicht normierte Schlagwörter; Gattungsbegriffe; etc.)

4. Wenn ja:

a) In welchem Zeitraum fand/findet die Erschließung statt?

b) Wie groß war/ist der Aufwand einer solchen Erschließung?

c) Würden Sie diesen Aufwand als lohnend bezeichnen? Könnte dieses Vorgehen bei der Erschließung als Modell für andere Bibliotheken dienen?

5.

a) Planen Sie, zukünftig eine Sacherschließung Ihrer Altbestände oder von Teilen Ihrer Altbestände durchzuführen bzw. die gegenwärtige Sacherschließung fortzuführen, evtl. sogar auszubauen, und wenn ja, in welcher Art?

b) Wenn nein: Aus welchen Gründen verzichten Sie auf die Inhaltliche Erschließung von Altbeständen?

6. Gibt es sonstiges, was Sie zur Thematik der inhaltlichen Sacherschließung von Altbeständen anmerken wollen? 


\section{Literaturverzeichnis}

AAD Gattungs- und Sachbegriffe (2005). Gemeinsamer Bibliotheksverbund (GBV). Online verfügbar unter http://aad.gbv.de/empfehlung/aad_gattung.pdf, zuletzt aktualisiert am 14.12.2005, zuletzt geprüft am 17.02.2008.

AG-SDD, AG Sammlung Deutsche Drucke - Home (2007). Online verfügbar unter http://www.ag-sdd.de/, zuletzt aktualisiert am 11.04.2007, zuletzt geprüft am 16.02.2008.

AG-SDD, Staatsbibliothek zu Berlin - Preußischer Kulturbesitz (2007). Online verfügbar unter http://www.ag-sdd.de/bibliotheken/spk.htm, zuletzt aktualisiert am 21.02.2007, zuletzt geprüft am 16.02.2008.

Angelus, Armin; Eichhorn-Berndt, Christine; Schnelling, Heiner (2000): Digitalisierung des Realkataloges (Hartwig-Katalog) der Universitäts- und Landesbibliothek Sachsen-Anhalt und seine Visualisierung im Internet. In: Bibliotheksdienst, Jg. 34, H. 3, S. 422-434. Online verfügbar unter http://bibliotheksdienst.zlb.de/2000/2000_03_Erschlie01.pdf, zuletzt geprüft am 23.02.2008.

ARK Sachkatalog der Staatsbibliothek zu Berlin - Preußischer Kulturbesitz (2008). Online verfügbar unter http://ark.staatsbibliothek-berlin.de/index.php, zuletzt geprüft am 17.02.2008.

ARK-Online (2008). Online verfügbar unter http://arkat.bsb-muenchen.de/start.php?offen=@@RK@@\&prung=RK\&sy=RK\&modus=PUB, zuletzt geprüft am 18.02.2008.

Arnold, Werner (1997): Zur klassifikatorischen Sacherschließung alter Drucke, vornehmlich am Beispiel der Herzog August Bibliothek Wolfenbüttel. In: Bibliothek. Forschung und Praxis, Jg. 21, H. 3, S. 333-338. Online verfügbar unter http://www.bibliothek-saur.de/1997_3/333-338.pdf, zuletzt geprüft am 16.02.2008.

b2i, Wissenschaftsportal (2008): Fabian-Handbuch: Home. Online verfügbar unter http://www.b2i.de/fabian, zuletzt geprüft am 16.02.2008.

BASE - Bielefeld Academic Search Engine (2008). Online verfügbar unter http://www.base-search.net/, zuletzt geprüft am 18.02.2008.

Basisklassifikation (2004). 3., erweiterte Ausgabe 2000. Überarbeitet durch die Facharbeitsgruppe Sacherschließung. Letzte Aktualisierung: 1. Ergänzungslieferung Dezember 2004. Gemeinsamer Bibliotheksverbund (GBV). Online verfügbar unter http://www.gbv.de/vgm/info/mitglieder/02Verbund/01Erschliessung/05Sacherschliessung/Sacherschliessung_0448.pdf, zuletzt geprüft am 17.02.2008. 
Bee, Guido; Karg, Helga (2007): Navigieren zwischen Schlagwort und Notation. CrissCross als Verbindung zwischen SWD und DDC. 96. Deutscher Bibliothekartag 2007. Veranstaltung vom 19.03.2007. Leipzig. Online verfügbar unter http://www.opusbayern.de/bib-info/volltexte/2007/381/pdf/LeipzigVortragstext.pdf, zuletzt geprüft am 18.02.2008.

Bertram, Jutta (2005): Einführung in die inhaltliche Erschließung. Grundlagen - Methoden - Instrumente. Würzburg: Ergon-Verl. (Content and communication, 2).

Block, Barbara (2006): Kooperative Neukatalogisierung. Eine neue Facette in der Zusammenarbeit der Verbünde. 95. Deutscher Bibliothekartag 2006. Veranstaltung vom 22.03.2006. Dresden. Online verfügbar unter http://www.gbv.de/vgm/info/biblio/01VZG/06Publikationen/2006/pdf/pdf_1806.pdf, zuletzt geprüft am 18.02.2008.

Block, Barbara (2007): Kooperative Neukatalogisierung als Standardverfahren in der verbundübergreifenden Zusammenarbeit. 96. Deutscher Bibliothekartag 2007. Veranstaltung vom 20.03.2007. Leipzig. Online verfügbar unter https://www.gbv.de/vgm/info/biblio/01VZG/06Publikationen/2007/pdf/pdf_2853.pdf, zuletzt geprüft am 18.02.2008.

Block, Barbara; Labner, Josef; Rusch, Beate (2007): Katalogisierung kooperativ gemacht. In: Bibliotheksdienst, Jg. 41, H. 2, S. 150-158. Online verfügbar unter http://www.zlb.de/aktivitaeten/bd_neu/heftinhalte2007/BD_Erschliessung_01_0207.pdf, zuletzt geprüft am 18.02.2008.

Bötte, Gerd-Josef (2004): VD 18: Vom bibliographischen Nachweis zum Digitalen Dokument. In: Schnelling, Heiner (Hg.): VD 18. Verzeichnis der im deutschen Sprachraum erschienenen Drucke des 18. Jahrhunderts. Beiträge eines DFG-Rundgesprächs in der Universitäts- und Landesbibliothek Sachsen-Anhalt in Halle (Saale), veranstaltet am 05.05.2004. Halle (Saale): Univ.- und Landesbibliothek Sachsen-Anhalt (Schriften zum Bibliotheks- und Büchereiwesen in Sachsen-Anhalt, 86), S. 95-109.

Bötte, Gerd-Josef (2005): Das deutsche Schrifttum des achtzehnten Jahrhunderts und seine bibliographische Verzeichnung - Perspektiven für ein VD18. Online verfügbar unter http://www.opus-bayern.de/bib-info/volltexte/2006/141/pdf/Boette-VD18_BTag2005-opusMS_pdf.pdf, zuletzt geprüft am 16.02.2008.

Bos, Jan (2007): Auskunft über die Sacherschließungspraxis im Short Title Catalogue Netherlands (STCN). E-Mail vom 22.05.2007 an Hannes Schwarzendorfer.

Braungart, Georg (2004): Kanon und Forschung: die Bedeutung der Erschließung für die Literaturwissenschaft. In: Schnelling, Heiner (Hg.): VD 18. Verzeichnis der im deutschen Sprachraum erschienenen Drucke des 18. Jahrhunderts. Beiträge eines DFGRundgesprächs in der Universitäts- und Landesbibliothek Sachsen-Anhalt in Halle (Saale), veranstaltet am 05.05.2004. Halle (Saale): Univ.- und Landesbibliothek Sachsen-Anhalt (Schriften zum Bibliotheks- und Büchereiwesen in Sachsen-Anhalt, 86), S. 43-48. 
Brinkhus, Gerd (2006): Der historische Altbestand in der modernen Bibliothek. Forum Bestandserhaltung. Online verfügbar unter http://www.uni-muenster.de/Forum-Bestandserhaltung/grundlagen/allg-brinkus.html, zuletzt aktualisiert am 31.05.2006, zuletzt geprüft am 16.02.2008.

Brinkhus, Gerd; Schibel, Wolfgang (2001): Historische Bibliotheksbestände im Abseits? In: Bibliotheksdienst, Jg. 35, H. 4, S. 427-438. Online verfügbar unter http://bibliotheksdienst.zlb.de/2001/01_04_03.pdf, zuletzt geprüft am 23.02.2008.

Buchanan, Brian (1989): Bibliothekarische Klassifikationstheorie. Übersetzt von Ute Reimer-Böhmer. München: Saur.

Bürger, Thomas (2004): Historische Drucke in Virtuellen Fachbibliotheken? Nutzen und Möglichkeiten sachlicher Sucheinstiege in das VD17 und VD18. In: Schnelling, Heiner (Hg.): VD 18. Verzeichnis der im deutschen Sprachraum erschienenen Drucke des 18. Jahrhunderts. Beiträge eines DFG-Rundgesprächs in der Universitäts- und Landesbibliothek Sachsen-Anhalt in Halle (Saale), veranstaltet am 05.05.2004. Halle (Saale): Univ.- und Landesbibliothek Sachsen-Anhalt (Schriften zum Bibliotheks- und Büchereiwesen in Sachsen-Anhalt, 86), S. 85-94.

CARMEN AP12 (2000). Crosskonkordanzen von Klassifikationen und Thesauri. Online verfügbar unter http://www.bibliothek.uni-regensburg.de/projects/carmen12/index.html.de, zuletzt aktualisiert am 18.02.2000, zuletzt geprüft am 18.02.2008.

Corsten, Sven (1985): Das Projekt zur Erschließung der historischen Buchbestände in Deutschland. In: Frankenberger, Rudolf; Habermann, Alexandra (Hg.): Literaturversorgung in den Geisteswissenschaften. 75. Deutscher Bibliothekartag in Trier 1985. Frankfurt am Main: Klostermann (Zeitschrift für Bibliothekswesen und Bibliographie : Sonderhefte, 43), S. 186-193.

Corsten, Severin; Bischoff, Bernhard (1987-2004): Lexikon des gesamten Buchwesens. LGB 2. 2., völlig neubearb. Aufl. Stuttgart: Hiersemann.

Dewey-Dezimalklassifikation (DDC) (2008). Unter Mitarbeit von Heidrun Alex. Deutsche Nationalbibliothek. Online verfügbar unter http://www.ddc-deutsch.de/, zuletzt aktualisiert am 25.01.2008, zuletzt geprüft am 17.02.2008.

DigiSyk-Benutzerversion (2008). Online verfügbar unter http://digisykben.wlb-stuttgart.de:8080/digisyk2ben/ben_syk_funktionen.php?indexsuche=1\&syk_root_id=10, zuletzt geprüft am 18.02.2008.

Dimitriadu, Valentina (1994): Der historische Standortkatalog und der Fachkatalog der Sächsischen Landesbibliothek Dresden. Diplomarbeit. Dresden.

Dreiländerkatalog (2006). hbz. Online verfügbar unter http://www.hbz-nrw.de/angebote/dlk/, zuletzt aktualisiert am 24.11.2006, zuletzt geprüft am 29.05.2007.

English Short Title Catalogue (2008). Online verfügbar unter http://estc.bl.uk/F/?func=file\&file_name=login-bl-list, zuletzt geprüft am 16.02.2008. 
Erste Planungen für ein Verzeichnis der im deutschen Sprachraum erschienenen Drucke des 18.Jahrhunderts (VD 18) (2004). In: Bibliotheksdienst, Jg. 38, H. 12, S. 1565-1571. Online verfügbar unter http://www.zlb.de/aktivitaeten/bd_neu/heftinhalte/heft9-1204/AltBuch1204.pdf, zuletzt geprüft am 16.02.2008.

Fabian, Bernhard (Hg.) (1992-2000): Handbuch der historischen Buchbestände in Deutschland. In Zusammenarbeit mit Severin Corsten ... Hrsg. von Bernhard Fabian. 27 Bände in 28 Bänden. Hildesheim: Olms-Weidmann.

Fabian, Bernhard (1983): Buch, Bibliothek und geisteswissenschaftliche Forschung. Zu Problemen der Literaturversorgung und der Literaturproduktion in der Bundesrepublik Deutschland. Göttingen: Vandenhoeck \& Ruprecht (Schriftenreihe der Stiftung Volkswagenwerk, 24).

Fabian, Bernhard (1985): Das Handbuch der historischen Buchbestände in der Bundesrepublik Deutschland und in West-Berlin. In: Zeitschrift für Bibliothekswesen und Bibliographie, Jg. 32, S. 379-388.

Fabian, Bernhard (2004): Brauchen wir ein Verzeichnis der deutschen Drucke des achtzehnten Jahrhunderts? In: Schnelling, Heiner (Hg.): VD 18. Verzeichnis der im deutschen Sprachraum erschienenen Drucke des 18. Jahrhunderts. Beiträge eines DFG-Rundgesprächs in der Universitäts- und Landesbibliothek Sachsen-Anhalt in Halle (Saale), veranstaltet am 05.05.2004. Halle (Saale): Univ.- und Landesbibliothek Sachsen-Anhalt (Schriften zum Bibliotheks- und Büchereiwesen in Sachsen-Anhalt, 86), S. 1-9.

Fietz, Rudolf (1992): Der alte Realkatalog der Landesbibliothek Oldenburg. Seine Entstehung und seine Systematik. In: Koolman, Egbert (Hg.): Ex Bibliotheca Oldenburgensi. Bibliothekarische Untersuchungen aus Anlaß des 200jährigen Bestehens der Landesbibliothek Oldenburg. Hrsg. von Egbert Koolman. Oldenburg: Holzberg (Schriften der Landesbibliothek Oldenburg, 26).

Flachmann, Holger (2004): Zur Effizienz bibliothekarischer Inhaltserschließung: Allgemeine Probleme und die Regeln für den Schlagwortkatalog (RSWK). In: Bibliotheksdienst, Jg. 38, H. 6, S. 745-791. Online verfügbar unter http://www.zlb.de/aktivitaeten/bd_neu/heftinhalte/heft9-1204/Erschliessung030604.pdf, zuletzt geprüft am 16.02.2008.

Frankenberger, Rudolf; Habermann, Alexandra (Hg.) (1985): Literaturversorgung in den Geisteswissenschaften. 75. Deutscher Bibliothekartag in Trier 1985. Frankfurt am Main: Klostermann (Zeitschrift für Bibliothekswesen und Bibliographie : Sonderhefte, 43).

Frankenberger, Rudolf; Haller, Klaus (Hg.) (2004): Die moderne Bibliothek. Ein Kompendium der Bibliotheksverwaltung. Herausgegeben von Rudolf Frankenberger und Klaus Haller. München: Saur. 
Fuhlrott, Rolf; Haller, Bertram (Hg.) (1979): Das Buch und sein Haus. Gerhard Liebers gewidmet zur Vollendung des 65. Lebensjahres am 23. Mai 1979. 2 Bände. Wiesbaden: Reichert.

Funke, Fritz (2006): Buchkunde. Die historische Entwicklung des Buches von der Keilschrift bis zur Gegenwart. 6., überarb. und erg. Aufl., Sonderausgabe. Wiesbaden: Albus im VMA-Verlag.

Gesamtverzeichnis des deutschsprachigen Schrifttums. (GV) 1700-1910. (19791987). Bearb. unter der Leitung von Peter Geils und Willi Gorzny. 160 Bände. München: Saur.

Gillitzer, Berthold; Weber, Andreas (2007): Des OPACs neue Kleider - vom Benutzerkatalog zur Suchmaschine. Neue Möglichkeiten für Bibliothekskataloge durch den Einsatz von Suchmaschinentechnologie. In: Bibliotheksforum Bayern, Jg. 1, H. 4, S. 218-221. Online verfügbar unter http://tinyurl.com/2hyqjq, zuletzt geprüft am 12.02.2008.

Hacker, Rupert (2000): Bibliothekarisches Grundwissen. 7., neu bearbeitete Auflage. München: Saur.

Haller, Klaus (Hg.) (1994): Regeln für die Katalogisierung alter Drucke. Erarbeitet von der Arbeitsgruppe des Deutschen Bibliotheksinstituts "RAK-WB und Alte Drucke". Herausgegeben und eingeleitet von Klaus Haller. Berlin: Dt. Bibliotheksinst. (Schriften der Deutschen Forschungsgemeinschaft).

Haller, Klaus (Hg.) (1995): Altbestandserschließung in wissenschaftlichen Bibliotheken. Ein Förderprogramm der Deutschen Forschungsgemeinschaft. Hrsg. von Klaus Haller ... [Mit Beitr. von Reinhard Altenhöner ...]. Berlin: Dt. Bibliotheksinst. (DBI-Materialien : Schriften der Deutschen Forschungsgemeinschaft, 143).

Haller, Klaus (1998): Katalogkunde. Eine Einführung in die Formal- und SacherschlieBung. 3., erw. Aufl. München: Saur.

Haller, Klaus; Fabian, Gudrun (2004): Bestandserschließung. In: Frankenberger, Rudolf; Haller, Klaus (Hg.): Die moderne Bibliothek. Ein Kompendium der Bibliotheksverwaltung. Herausgegeben von Rudolf Frankenberger und Klaus Haller. München: Saur, S. 222-261.

Hartwieg, Ursula (2004): Projekt "Verzeichnis der deutschen Drucke des 18.Jahrhunderts". In: Zeitschrift für Bibliothekswesen und Bibliographie, Jg. 51, H. 4, S. 222-228.

Hausinger, Angela (2007): Auskunft über die Sacherschließung des Zeitsegmentes 1801 - 1870 innerhalb der Sammlung Deutscher Drucke. E-Mail vom 15.05.2007 an Hannes Schwarzendorfer.

Hilfe für Anna Amalia (2007). Online verfügbar unter http://www.anna-amalia-bibliothek.de/de/afterbrand.html, zuletzt aktualisiert am 14.12.2007, zuletzt geprüft am 17.02.2008. 
Hoinkis, Gudrun (1999): Bisherige Erschließung der Drucke des 17. Jahrhunderts (VD17). In: Bibliotheksdienst, Jg. 33, H. 11, S. 1874-1885. Online verfügbar unter http://bibliotheksdienst.zlb.de/1999/1999_11_Erschlie02.pdf, zuletzt geprüft am 16.02.2008.

Holbach, Werner (2006): Retrokonversion und Retrokatalogisierung in den Bayerischen Staatlichen Bibliotheken. In: Bibliotheksforum Bayern, Jg. 34, H. 3, S. 206-228.

Hörth, Claudius (2002): Klassifikation für Digitale Bibliotheken: Ein Lösungsvorschlag für die digitale Bibliothek „Information und Medien“ der Hochschule der Medien, Stuttgart. Diplomarbeit. Betreut von Margarete Payer. Stuttgart. Hochschule der Medien, Fachbereich Information und Kommunikation. Online verfügbar unter http://www.fkdigbib.de/downloads/diplomarbeit_C_Hoerth.pdf, zuletzt geprüft am 23.02.2008.

Hrusa, Hans (Hg.) (2005): Bibliothek, Technik, Recht. Festschrift für Peter Kubalek zum 60. Geburtstag. Wien: Manz.

Impe, Steven van; Rossem, Stijn van; Proot, Goran (2005): Handleiding voor de Short Title Catalogus Vlaanderen. Tweede, herziene uitgave. Antwerpen: Stadsbibliotheek Antwerpen (Armarium, 1).

InfoGuide. UB Bayreuth (2008). Online verfügbar unter http://opac.unibayreuth.de:8080/InfoGuideClient/initSearch.do, zuletzt geprüft am 18.02.2008.

INKA (2008). Online verfügbar unter http://www.inka.uni-tuebingen.de/?bib=stu, zuletzt geprüft am 16.02.2008.

Jefcoate, Graham (2004): Zur quantitativen Präzisierung des Volumens der Literatur des 18. Jahrhunderts - Probleme und Lösungen im deutschsprachigen Raum und internationalen Bereich. In: Schnelling, Heiner (Hg.): VD 18. Verzeichnis der im deutschen Sprachraum erschienenen Drucke des 18. Jahrhunderts. Beiträge eines DFG-Rundgesprächs in der Universitäts- und Landesbibliothek Sachsen-Anhalt in Halle (Saale), veranstaltet am 05.05.2004. Halle (Saale): Univ.- und Landesbibliothek Sachsen-Anhalt (Schriften zum Bibliotheks- und Büchereiwesen in Sachsen-Anhalt, 86), S. 61-74.

Kaltwasser, Franz Georg (1985): Erschließung alter Buchbestände in Bibliotheken der Bundesrepublik Deutschland. In: Frankenberger, Rudolf; Habermann, Alexandra (Hg.): Literaturversorgung in den Geisteswissenschaften. 75. Deutscher Bibliothekartag in Trier 1985. Frankfurt am Main: Klostermann (Zeitschrift für Bibliothekswesen und Bibliographie : Sonderhefte, 43), S. 163-185.

Kirchliches Buch- und Bibliothekswesen. Jahrbuch 2004 (2005). Rottenburg am Neckar: Diözesanbibliothek der Diözese Rottenburg-Stuttgart.

KOBV - KOBV-Suchmaschine (2007). Kooperativer Bibliotheksverbund Berlin-Brandenburg. Online verfügbar unter http://www.kobv.de/deutsch/content/partner_info/kobvsuchmaschine.htm, zuletzt geprüft am 23.02.2008. 
Koolman, Egbert (Hg.) (1992): Ex Bibliotheca Oldenburgensi. Bibliothekarische Untersuchungen aus Anlaß des 200jährigen Bestehens der Landesbibliothek Oldenburg. Hrsg. von Egbert Koolman. Oldenburg: Holzberg (Schriften der Landesbibliothek OIdenburg, 26).

Köstädt, Peter (2006): FAST-Anwendungen und Dienstleistungen des hbz. Veranstaltung vom 31.05.06, aus der Reihe "Fast-Workshop". Köln. Veranstalter: hbz. Online verfügbar unter http://www.hbz-nrw.de/dokumentencenter/produkte/dlk/aktuell/vortraege/FAST-Workshop/FAST-Workshop_pk.pdf, zuletzt geprüft am 18.02.2008.

Kowalewski, Isabel (1999): Barockliteratur online im Internet - Das Verzeichnis der im deutschen Sprachraum erschienenen Drucke des 17. Jahrhunderts (VD 17). Köln: Fachhochschule Köln, Fachbereich Bibliotheks- und Informationswesen (Kölner Arbeitspapiere zur Biblioheks- und Informationswissenschaft, 21).

Krabbe, Wilhelm; Luther, Wilhelm Martin (1953): Lehrbuch der Bibliotheksverwaltung. Stuttgart: Hiersemann. Online verfügbar unter http://www.ib.hu-berlin.de/ wumsta/kralu/kul4.html, zuletzt geprüft am 17.02.2008.

Kratzsch, Konrad; Seifert, Siegfried (Hg.) (1992): Historische Bestände der Herzogin-Anna-Amalia-Bibliothek zu Weimar. Beiträge zu ihrer Geschichte und Erschließung ; mit Bibliographie. München: Saur (Literatur und Archiv, 6).

Krause, Jürgen (1996): Informationserschließung und -bereitstellung zwischen Deregulation, Kommerzialisierung und weltweiter Vernetzung - Schalenmodel -. InformationsZentrum Sozialwissenschaften. (IZ-Arbeitsbericht, Nr. 6). Online verfügbar unter http://www.gesis.org/Publikationen/Berichte/IZ_Arbeitsberichte/pdf/ab6.pdf, zuletzt geprüft am 18.02.2008.

Krause, Jürgen (2000): Sacherschließung in virtuellen Bibliotheken. Standardisierung versus Heterogenität. In: Rützel-Banz, Margit (Hg.): Grenzenlos in die Zukunft. 89. Deutscher Bibliothekartag in Freiburg im Breisgau 1999. Frankfurt am Main: Klostermann (Zeitschrift für Bibliothekswesen und Bibliographie : Sonderhefte, 77), S. 202212.

Krause, Jürgen (2004a): Standardisierung und Heterogenität. In: Kuhlen, Rainer; Seeger, Thomas; Strauch, Dietmar (Hg.): Grundlagen der praktischen Information und Dokumentation. Begr. von Klaus Laisiepen ... Band 1: Handbuch zur Einführung in die Informationswissenschaft und -praxis. München: Saur, S. 635-641.

Krause, Jürgen (2004b): Konkretes zur These, die Standardisierung von der Heterogenität her zu denken. In: Zeitschrift für Bibliothekswesen und Bibliographie, Jg. 51, H. 2, S. 76-89.

Krause, Jürgen; Schwänzl, Roland (1998): Inhaltserschließung: Formale Beschreibung, Identifikation und Retrieval, MetaDaten, Vernetzung. Online verfügbar unter http://www.mathematik.uni-osnabrueck.de/projects/slot3/workshop98II/KS.html, zuletzt aktualisiert am 30.04.1998, zuletzt geprüft am 18.02.2008. 
Kronenberg, Hermann (2006): Suchmaschinentechnologie versus klassischer Bibliotheks-OPAC. Am Beispiel des Dreiländerkatalogs. Online verfügbar unter http://verbundtag.obvsg.at/documents/2006/vbt06_dreilaenderkatalog_hk.pdf, zuletzt aktualisiert am 07.06.2006, zuletzt geprüft am 23.02.2008.

Kuberek, Monika (1999): Dublettenbehandlung (Match- und Merge-Verfahren) in der KOBV-Suchmaschine. Grundlagen. Konrad-Zuse-Zentrum für Informationstechnik Berlin. (Preprint, SC 99-16). Online verfügbar unter http://www.zib.de/Publications/Reports/SC-99-16.pdf, zuletzt geprüft am 18.02.2008.

Kuhlen, Rainer; Seeger, Thomas; Strauch, Dietmar (Hg.) (2004a): Grundlagen der praktischen Information und Dokumentation. Begr. von Klaus Laisiepen ... Band 1: Handbuch zur Einführung in die Informationswissenschaft und -praxis. München: Saur.

Kuhlen, Rainer; Seeger, Thomas; Strauch, Dietmar (Hg.) (2004b): Grundlagen der praktischen Information und Dokumentation. Begr. von Klaus Laisiepen... Band 2: Glossar. München: Saur.

KVK Karlsruher Virtueller Katalog (2008). Universitätsbibliothek Karlsruhe. Online verfügbar unter http://www.ubka.uni-karlsruhe.de/kvk.html, zuletzt aktualisiert am 29.01.2008, zuletzt geprüft am 17.02.2008.

Lapp, Erdmute (1989): Katalogsituation der Altbestände (1501 - 1850) in Bibliotheken der Bundesrepublik Deutschland einschließlich Berlin (West). Eine Studie im Auftrag der Deutschen Forschungsgemeinschaft. Berlin: Dt. Bibliotheksinst. (DBI-Materialien, 82).

Lewandowski, Dirk (2006): Suchmaschinen als Konkurrenten der Bibliothekskataloge. Wie Bibliotheken ihre Angebote durch Suchmaschinentechnologie attraktiver und durch Öffnung für die allgemeinen Suchmaschinen populärer machen können. In: Zeitschrift für Bibliothekswesen und Bibliographie, Jg. 53, H. 2, S. 71-78. Online verfügbar unter http://www.durchdenken.de/lewandowski/doc/ZFBB_Preprint.pdf, zuletzt geprüft am 18.02.2008.

Leyh, Georg (1961): Handbuch der Bibliothekswissenschaft. Begründet von Fritz Milkau. Band 2: Bibliotheksverwaltung. 2., verm. und verb. Auflage. Wiesbaden: Harrassowitz.

Library of Congress Subject Headings Principles and Policies (2001). Online verfügbar unter http://www.itsmarc.com/crs/shed0014.htm, zuletzt aktualisiert am 28.02.2001, zuletzt geprüft am 16.02.2008.

Löffler, Karl; Umstätter, Walther; Wagner-Döbler, Roland (2005): Einführung in die Katalogkunde. Vom Zettelkatalog zur Suchmaschine. Dritte Auflage des Werkes von Karl Löffler. Völlig neu bearbeitet von Walther Umstätter und Roland Wagner-Döbler. Stuttgart: Hiersemann.

Lorenz, Bernd (1998): Klassifikatorische Sacherschließung. Eine Einführung. Wiesbaden: Harrassowitz (Bibliotheksarbeit, 5). 
Lorenz, Bernd (2003): Systematische Aufstellung in Vergangenheit und Gegenwart. Wiesbaden: Harrassowitz (Beiträge zum Buch- und Bibliothekswesen, 45).

Lossau, Norbert (2004): Suchmaschinentechnologie und Digitale Bibliotheken - Bibliotheken müssen das wissenschaftliche Internet erschließen. In: Zeitschrift für Bibliothekswesen und Bibliographie, Jg. 51, H. 5-6, S. 284-294.

Lügger, Joachim (2000): Über Suchmaschinen, Verbünde und die Integration von Informationsangeboten. Teil I. KOBV-Suchmaschine und Math-Net. Konrad-Zuse-Zentrum für Informationstechnik Berlin. (ZIB-Report, 00-23). Online verfügbar unter http://www.zib.de/Publications/Reports/ZR-00-23.pdf, zuletzt geprüft am 18.02.2008.

Lülfing, Daniela; Kessen, Kathrin (Hg.) (2006): "Geld ist rund und rollt weg, aber Bildung bleibt". 94. Deutscher Bibliothekartag in Düsseldorf 2005. Frankfurt am Main: Klostermann (Zeitschrift für Bibliothekswesen und Bibliographie : Sonderhefte, 89).

May, Simon (2007): Auskunft über die Sacherschließungspraxis im English Short Title Catalogue (ESTC). E-Mail vom 15.05.2007 an Hannes Schwarzendorfer.

Mayr, Philipp; Walter, Anne-Kathrin (2007): Zum Stand der Heterogenitätsbehandlung in vascoda: Bestandsaufnahme und Ausblick. GESIS / Informationszentrum Sozialwissenschaften (IZ). Bonn. Online verfügbar unter http://www.ib.huberlin.de/ mayr/arbeiten/BID2007-Mayr-Walter.pdf, zuletzt geprüft am 18.02.2008.

Meyer, Hans-Martin (1998): Das "Verzeichnis der im deutschen Sprachraum erschienenen Drucke des 17. Jahrhunderts". Analyse des VD17 und Entwicklung eines Konzeptes zur Einbindung in Verbundkataloge. Diplomarbeit. Betreut von Margarete Payer. Stuttgart. Fachhochschule Stuttgart - Hochschule für Bibliotheks- und Informationswesen.

Mittelbach, Jens; Probst, Michaela (2006): Maschinelle Indexierung in der Sacherschließung wissenschaftlicher Bibliotheken. In: Bibliothek. Forschung und Praxis, Jg. 30, H. 2, S. 168-176. Online verfügbar unter http://www.bibliotheksaur.de/2006_2/168-176.pdf, zuletzt geprüft am 23.02.2008.

Möncke, Gisela (2004): Das "Verzeichnis der im deutschen Sprachbereich erschienenen Drucke des 16.Jahrhunderts" (VD 16) als Teil einer deutschen retrospektiven Nationalbibliographie. In: Zeitschrift für Bibliothekswesen und Bibliographie, Jg. 51, H. 4, S. 207-212.

Müller, Wolfgang (1990): Die Drucke des 17. Jahrhunderts im deutschen Sprachraum. Untersuchungen zu ihrer Verzeichnung in einem VD17. Im Auftrag der Deutschen Forschungsgemeinschaft vorgelegt von Wolfgang Müller. Wiesbaden: Harrassowitz (Beiträge zum Buch- und Bibliothekswesen, 31).

Münster, Westfälische Wilhelms-Universität; Institut für Buchwissenschaft und Textforschung (2007): IBT - Handbuch der historischen Buchbestände. Westfälische Wilhelms-Universität Münster. Online verfügbar unter https://www.unimuenster.de/Buchwiss/Forschen/Handbuch_der_historischen_Buchbestaende.html, zuletzt aktualisiert am 07.11.2007, zuletzt geprüft am 16.02.2008. 
Niedersächsische Staats- und Universitätsbibliothek (2008). bandrksyst. Online verfügbar unter http://opac.sub.uni-goettingen.de/DB=1/BANDRKSYST, zuletzt geprüft am 17.02.2008.

Nohr, Holger (2004): Theorie des Information Retrieval II: Automatische Indexierung. In: Kuhlen, Rainer; Seeger, Thomas; Strauch, Dietmar (Hg.): Grundlagen der praktischen Information und Dokumentation. Begr. von Klaus Laisiepen ... Band 1: Handbuch zur Einführung in die Informationswissenschaft und -praxis. München: Saur, S. 215-225.

Nohr, Holger (2005): Grundlagen der automatischen Indexierung. Ein Lehrbuch. 3. Auflage. Berlin: Logos-Verl.

Oberhauser, Otto (2005): Automatisches Klassifizieren und Bibliothekskataloge. In: Hrusa, Hans (Hg.): Bibliothek, Technik, Recht. Festschrift für Peter Kubalek zum 60. Geburtstag. Wien: Manz, S. 119-131.

Oberhauser, Otto; Gödert, Winfried (2005): Automatisches Klassifizieren. Entwicklungsstand - Methodik - Anwendungsbereiche. Mt einem Vorwort von Winfried Gödert. Frankfurt am Main: Lang (Europäische Hochschulschriften : Reihe XLI ; Informatik, 43).

Oettingen-Wallersteinsche Bibliothek (2007). Online verfügbar unter http://www.bibliothek.uni-augsburg.de/sondersammlungen/oettingen_wallerstein/, zuletzt aktualisiert am 31.07.2007, zuletzt geprüft am 23.02.2008.

OPACplus. Bayerische Staatsbibliothek (2008). Online verfügbar unter https://opacplus.bsb-muenchen.de/InfoGuideClient/start.do?Login=opacext\&BaseURL, zuletzt geprüft am 18.02.2008.

Peitz, Peter (1997): Erschließung älterer Drucke. Was ist nötig, was ist möglich? In: ProLibris, Jg. 2, H. 2, S. 99-103.

Proot, Goran (2005): Ergebnisse des Short Title Catalogus Vlaanderen: Die erste Phase (2000-2003): Kirchliches Buch- und Bibliothekswesen. Jahrbuch 2004. Rottenburg am Neckar: Diözesanbibliothek der Diözese Rottenburg-Stuttgart, S. 227-249.

Proot, Goran (2007): Auskunft über die Sacherschließungspraxis im Short Title Catalogue Vlandeeren (STCV). E-Mail vom 14.05.2007 an Hannes Schwarzendorfer.

Raabe, Paul (1979): Das alte und kostbare Buch - eine bibliothekarische Zukunftsaufgabe. In: Fuhlrott, Rolf; Haller, Bertram (Hg.): Das Buch und sein Haus. Gerhard Liebers gewidmet zur Vollendung des 65. Lebensjahres am 23. Mai 1979. 2 Bände. Wiesbaden: Reichert, S. 164-188.

Raabe, Paul (1988): Die Bibliothek und die alten Bücher. Über das Erhalten, ErschlieBen und Erforschen historischer Bestände. Vortrag an der Universität Augsburg am 24. Juni 1987. Augsburg: Universität (Augsburger Universitätsreden, 11). 
RBMS Controlled Vocabularies. Genre Terms (2007). Online verfügbar unter http://www.rbms.info/committees/bibliographic_standards/controlled_vocabularies/genre/alphabetical_list.htm, zuletzt aktualisiert am 30.08.2007, zuletzt geprüft am 16.02.2008.

Reifegerste, Matthias (2007): Der systematische Katalog des Altbestandes 1500 -1967. Überarbeitete Fassung des Vortrags "Neues zum alten Freiburger SyKa 15001967. Eine Projektskizze". 31. Jahrestagung der Gesellschaft für Klassifikation. Veranstaltung vom 07.03.2007. Freiburg.

Riplinger, Thomas (2004): Die Bedeutung der Methode Eppelsheimer für Theorie und Praxis der bibliothekarischen und der dokumentarischen Sacherschließung. In: Bibliothek. Forschung und Praxis, Jg. 28, H. 2, S. 252-262. Online verfügbar unter http://www.bibliothek-saur.de/2004_2/252-262.pdf, zuletzt geprüft am 17.02.2008.

Rösch, Hermann (2005): Lehrveranstaltung Allgemeine Informationsmittel. Begleitende Informationen. Erarbeitet unter Mitarbeit von Sonja Härkönen. Online verfügbar unter http://www.fbi.fh-koeln.de/institut/personen/roesch/Material_Roesch/Informationsmittel/Inhalt.htm\#inhalt7.3, zuletzt aktualisiert am 26.01.2005, zuletzt geprüft am 16.02.2008.

RSWK (1998): Regeln für den Schlagwortkatalog. 3., überarb. und erw. Aufl. Berlin.

Online verfügbar unter

http://deposit.ddb.de/ep/netpub/89/96/96/967969689/_data_stat/www.dbi-

berlin.de/dbi_pub/einzelpu/regelw/rswk/rswk_00.htm, zuletzt geprüft am 16.02.2008.

Rückert, Ingrid (2003): Der Schrettinger-Katalog und der Alte Realkatalog. Zweihundert Jahre Sacherschließung an der Bayerischen Staatsbibliothek. München: Selbstverlag.

Rückert, Ingrid; Groß, Matthias (2003): Der Alte Realkatalog der Bayerischen Staatsbibliothek und sein neues Online-Register. In: Bibliotheksforum Bayern, Jg. 31, H. 3, S. 214-223.

Rützel-Banz, Margit (Hg.) (2000): Grenzenlos in die Zukunft. 89. Deutscher Bibliothekartag in Freiburg im Breisgau 1999. Frankfurt am Main: Klostermann (Zeitschrift für Bibliothekswesen und Bibliographie : Sonderhefte, 77).

RVK-Online (2008). Online verfügbar unter http://www.bibliothek.uniregensburg.de/rvko_neu/, zuletzt geprüft am 17.02.2008.

Schnelling, Heiner (Hg.) (2004): VD 18. Verzeichnis der im deutschen Sprachraum erschienenen Drucke des 18. Jahrhunderts. Beiträge eines DFG-Rundgesprächs in der Universitäts- und Landesbibliothek Sachsen-Anhalt in Halle (Saale), veranstaltet am 05.05.2004. Halle (Saale): Univ.- und Landesbibliothek Sachsen-Anhalt (Schriften zum Bibliotheks- und Büchereiwesen in Sachsen-Anhalt, 86).

Schottenloher, Karl (1956): Das alte Buch. Dritte Auflage. Mit 162 Abbildungen. Braunschweig: Klinkhardt \& Biermann (Bibliothek für Kunst- und Antiquitätenfreunde, 14). 
Seifert, Siegfried (1992): „Niemand wird läugnen, daß ein Real-Catalog das Fundament einer jeden Bibliotheks-Anstalt sey". Bemerkungen zum historischen Realkatalog der Weimarer Republik. In: Kratzsch, Konrad; Seifert, Siegfried (Hg.): Historische Bestände der Herzogin-Anna-Amalia-Bibliothek zu Weimar. Beiträge zu ihrer Geschichte und Erschließung ; mit Bibliographie. München: Saur (Literatur und Archiv, 6).

Short Title Catalogue Netherlands (2008). Online verfügbar unter http://www.kb.nl/stcn/sten-en.html, zuletzt geprüft am 16.02.2008.

Short Title Catalogus Vlaanderen (2005). Online verfügbar unter http://www.stcv.be/, zuletzt aktualisiert am 14.02.2005, zuletzt geprüft am 16.02.2008.

Siegert, Reinhart (2004): Defizite bibliothekarischer Kataloge aus Sicht der Forschung. In: Schnelling, Heiner (Hg.): VD 18. Verzeichnis der im deutschen Sprachraum erschienenen Drucke des 18. Jahrhunderts. Beiträge eines DFG-Rundgesprächs in der Universitäts- und Landesbibliothek Sachsen-Anhalt in Halle (Saale), veranstaltet am 05.05.2004. Halle (Saale): Univ.- und Landesbibliothek Sachsen-Anhalt (Schriften zum Bibliotheks- und Büchereiwesen in Sachsen-Anhalt, 86), S. 11-28.

Sieglerschmidt, Jörn (Hg.) (2007): Regelwerke für die Sacherschliessung: sexy oder uncool? Herausgegeben von Jörn Sieglerschmidt. Workshop am 10. November 2004. Electronic imaging and the visual arts. Berlin 10.-12. November 2004. Online verfügbar unter http://www2.bsz-bw.de/cms/museen/musis/publ/eva2004, zuletzt aktualisiert am 22.08.2007, zuletzt geprüft am 23.02.2008.

Stäcker, Thomas (2004): VD 17 - mehr als eine Zwischenbilanz. In: Zeitschrift für Bibliothekswesen und Bibliographie, Jg. 51, H. 4, S. 213-221.

Stumpf, Gerhard (2002): Online-Klassifikation und Klassifikation im Online-Katalog Alternativen für die RVK? 92. Deutscher Bibliothekartag. Veranstaltung vom 12.04.2002. Augsburg. Online verfügbar unter http://www2.bibliothek.uniaugsburg.de/allg/swk/Online-Klassifikation.html, zuletzt geprüft am 18.02.2008.

Stumpf, Gerhard (2002): Einführung in die verbale Inhaltserschließung für Bibliotheken. Kurzfassung des Skripts zum Kompaktkurs Juni 2002.

Suckow, Ninon; Klarkowski, Werner (2008): Gesamtkatalog der Wiegendrucke. Staatsbibliothek zu Berlin (SBB). Online verfügbar unter http://www.gesamtkatalogderwiegendrucke.de/, zuletzt aktualisiert am 16.01.2008, zuletzt geprüft am 16.02.2008.

Syré, Ludger (1987): Altbestandserfassung in wissenschaftlichen Bibliotheken der Bundesrepublik Deutschland. Berlin: Dt. Bibliotheksinst. (DBI-Materialien, 67).

Systematischer Standortkatalog SLUB (2003). Online verfügbar unter http://image.slub-dresden.de/de/Inc_txt/stok/index.html, zuletzt aktualisiert am 16.11.2003, zuletzt geprüft am 18.02.2008.

The Library of Congress (2008). Online verfügbar unter http://www.loc.gov/index.html, zuletzt geprüft am 16.02.2008. 
UB-Freiburg Systematiken (2008). Online verfügbar unter http://matterhorn-ng.ub.unifreiburg.de/SystWebS/systematik-

webservice. php?ctrl=showroot\&thessi=AB\&signatur=\&css=css3, zuletzt geprüft am 18.02.2008.

ULB Halle: Image-Kataloge (2001). Online verfügbar unter http://zkat.bibliothek.unihalle.de/, zuletzt aktualisiert am 26.04.2001, zuletzt geprüft am 18.02.2008.

Umlauf, Konrad (2006): Einführung in die bibliothekarische Klassifikationstheorie und -praxis. Mit Übungen. Institut für Bibliotheks- und Informationswissenschaft der Humboldt-Universität zu Berlin. (Berliner Handreichungen zur Bibliotheks- und Informationswissenschaft, 67). Online verfügbar unter http://www.ib.hu-berlin.de/\%7Ekumlau/handreichungen/h67/\#rvk, zuletzt aktualisiert am 20.12.2006, zuletzt geprüft am 17.02.2008.

Universitätsbibliothek Augsburg (2006): Internet-Informationen zur SacherschlieBung. Online verfügbar unter http://hpbib4.bibliothek.uni-

augsburg.de/allg/swk/sacher_allg.html, zuletzt aktualisiert am 07.09.2006, zuletzt geprüft am 23.02.2008.

Uns ist in alten Mären ... Das Nibelungenlied und seine Welt (2008). Badische Landesbibliothek. Online verfügbar unter http://www.blb-

karlsruhe.de/blb/blbhtml/aktuelles/nibelungen.html, zuletzt aktualisiert am 02.01.2008, zuletzt geprüft am 24.02.2008.

Verzeichnis des deutschsprachigen Schrifttums des 16. Jahrhunderts (2008). Online verfügbar unter http://www.vd16.de/, zuletzt geprüft am 16.02.2008.

Verzeichnis der Drucke des 17. Jahrhunderts (2007). Online verfügbar unter http://www.vd17.de/, zuletzt aktualisiert am 11.04.2007, zuletzt geprüft am 16.02.2008.

Verzeichnis der Drucke des 17. Jahrhunderts: Bibliographische Beschreibung (2007). Online verfügbar unter http://www.vd17.de/bibliographB.html, zuletzt aktualisiert am 24.04.2007, zuletzt geprüft am 16.02.2008.

Verzeichnis der Drucke des 17. Jahrhunderts: Gattungsbegriffe (2007). Online verfügbar unter http://www.vd17.de/gattungsbegriffe.html, zuletzt aktualisiert am 11.04.2007, zuletzt geprüft am 16.02.2008.

Vinzent, Otwin (1992): Katalogsituation der Altbestände (1501 - 1850) in Bibliotheken der neuen Bundesländer. Eine Studie im Auftrag der Deutschen Forschungsgemeinschaft. Berlin: Dt. Bibliotheksinst. (DBI-Materialien : Schriften der Deutschen Forschungsgemeinschaft, 116).

Weber, Andreas (2007): Suchmaschinentechnologie (FAST): Neue Möglichkeiten für den Benutzer. Veranstaltung vom 14.12.2007. München. Online verfügbar unter http://www.bib-bvb.de/vk2006/FAST_Weber_Verkundk_2006.pdf, zuletzt geprüft am 18.02.2008. 
Williamson, David (2007): Auskunft über die Sacherschließungspraxis in der Library of Congress (LOC). E-Mail vom 15.05.07 an Hannes Schwarzendorfer.

Wittmann, Reinhard (1991): Geschichte des deutschen Buchhandels. Ein Überblick. München: Beck.

Zimmermann, Harald (2003): Möglichkeiten einer computergestützten SacherschlieBung. Vortrag auf der 27. Jahrestagung der Gesellschaft für Klassifikation im "Bibliothekarischen Programm" an der Brandenburgischen Technischen Universität in Cottbus am 11. 3. 2003. Online verfügbar unter http://archiv.tuchemnitz.de/pub/2003/0066/data/zimmermann_saar.pdf, zuletzt geprüft am 23.02.2008. 